\title{
Iron(III)-Mediated Bicyclization of 1,2-Allenyl Aryl Ketones: Assembly of Indanone-Fused Polycyclic Scaffolds and Dibenzo[a,e]pentalene Derivatives
}

Maozhong Miao*, Mengchao Jin, Panpan Chen, Lei Wang, Shouzhi Zhang, and Hongjun Ren* Department of Chemistry, Zhejiang Sci-Tech University, Hangzhou, Zhejiang 310018 (P. R. China) mmzok@zstu.edu.cn; renhjtaizhou@163.com

\section{Supporting Information \\ List of contents}

1. General methods

S2

2. Procedure and experiment data for indanone-fused polycyclic compounds 2

S2-S15

3. Access to unsymmetrical dibenzo[a,e]pentalenes $\mathbf{4}$ from $\mathbf{2}$

S15-S23

4. Diverse transformations of $\mathbf{2}$ for the synthesis of 3D-polycyclic compounds 5-10 S23-S30

5. Emission spectra and density functional theory (DFT) bandgap calculation

S30-S43

6. Preparation of allenyl ketones $\mathbf{1}$

S43-S52

7. X-ray diffraction analysis of 2p, 2y, 5a, 9, and $\mathbf{1 0}$

S53-S59

8. Copies of ${ }^{1} \mathrm{H}$ NMR and ${ }^{13} \mathrm{C}$ NMR

S60-S181 


\section{General Methods.}

NMR spectra were recorded on a Bruker AV-400 MHz spectrometer. The ${ }^{1} \mathrm{H}$ NMR (400 MHz) chemical shifts were reported in parts per million $(\delta)$ relative to internal standard TMS $(0 \mathrm{ppm})$ in $\mathrm{CDCl}_{3}$ and solvent signals $(2.50 \mathrm{ppm})$ in DMSO- $d_{6}$. The coupling constants, $J$ values were reported in Hertz (Hz). The ${ }^{13} \mathrm{C}$ NMR (100 MHz) chemical shifts were referenced to the internal solvent signals (77.0 ppm in $\mathrm{CDCl}_{3}$, $39.52 \mathrm{ppm}$ in DMSO- $\left.d_{6}\right)$. High-resolution mass spectra (HRMS) were recorded on a Waters TOFMS GCT Premier using ESI ionization. UV-Vis spectra were taken on a HITACHI U3010 Spectrophotometer. Fluorescence measurements were performed on an Agilent Cary Eclipse Fluorescence Spectrophotometer. Melting points were measured with WRR digital point apparatus and not corrected. All commercial reagents were used without additional purification and solvents were dried by standard methods when necessary. The anhydrous $\mathrm{FeCl}_{3}(\mathrm{AR})$ was purchased from Aladdin Reagent Company, Inc. Petroleum ether referred to the fraction with boiling point in the range $60-90^{\circ} \mathrm{C}$. All reactions were monitored by TLC with GF 254 silica gel coated plates. Flash column chromatography was carried out using 200-300 mesh silica gel. The materials 1,3,3-triarylpropyne ${ }^{1}, \quad$ 2-methyl-1,4,4-triphenylbuta-2,3-dien-1-one $\quad \mathbf{2 o}^{2} \quad$ and 4-(4-isobutylphenyl)-2-methyl-1-phenylpenta-2,3-dien-1-one $2 \mathbf{z}^{2}$ was prepared according to the literature procedure.

\section{Procedure and experiment data for indanone-fused polycyclic compounds 2}

\section{(a) General procedure:}
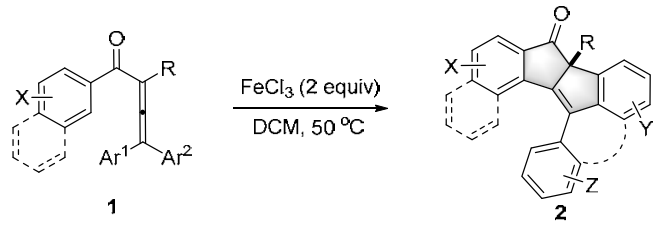

The solution of tetra-substituted allenyl ketones 1 ( $0.2 \mathrm{mmol}, 1.0$ equiv) in $2 \mathrm{~mL}$ of DCM was added 
$\mathrm{FeCl}_{3}$ (0.4 mmol, 2.0 equiv) in the open air. Then the vessel was sealed and submerged in an oil bath preheated to $50{ }^{\circ} \mathrm{C}$ for $12 \mathrm{~h}$. After completion of the reaction, the mixture was quenched by adding 10 $\mathrm{mL}$ of $\mathrm{H}_{2} \mathrm{O}$ and extracted with EtOAc $(3 \times 10 \mathrm{~mL})$. The combined organic phase was washed with $\mathrm{H}_{2} \mathrm{O}(3 \times 10 \mathrm{~mL})$, dried over anhydrous $\mathrm{Na}_{2} \mathrm{SO}_{4}$, concentrated in vacuo and purified by flash silica gel chromatography to afford indanone-fused polycycles 2.

\section{(b) Experiment data:}

\section{4b,10-diphenylindeno[2,1-a]inden-5(4bH)-one (2a)}

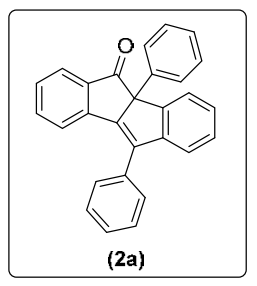

The reaction of 1,2,4,4-tetraphenylbuta-2,3-dien-1-one 1a (74.8 mg, 0.2 mmol, 1.0 equiv), $\mathrm{FeCl}_{3}$ (65 mg, $0.4 \mathrm{mmol}, 2.0$ equiv), in $2 \mathrm{~mL}$ of $\mathrm{DCM}$ at $50{ }^{\circ} \mathrm{C}$ for $12 \mathrm{~h}$ afforded 2a (64.7 $\mathrm{mg}, 87 \%)$ as a yellow solid; $5.580 \mathrm{~g}$ (15 mmol) scale reaction:

The reaction of 1,2,4,4-tetraphenylbuta-2,3-dien-1-one 1a (5.580 g, 15 mmol, 1.0 equiv), $\mathrm{FeCl}_{3}$ (4.860 g, $30 \mathrm{mmol}, 2.0$ equiv), in $150 \mathrm{~mL}$ of DCM at $50{ }^{\circ} \mathrm{C}$ for $12 \mathrm{~h}$ afforded 2a (4.456 g, 80\%) as a yellow solid; m.p. $167-168{ }^{\circ} \mathrm{C}$ (Petroleum ether/EtOAc); $\mathrm{R} f=0.45$ (Petroleum ether/EtOAc = 10/1); ${ }^{1} \mathrm{H}$ NMR (400 MHz, $\mathrm{CDCl}_{3}$ ): $\delta$ 7.77-7.72 (m, 3H), 7.71-7.67 (m, 3H), 7.64 (d, $\left.J=8.0 \mathrm{~Hz}, 1 \mathrm{H}\right), 7.57$ (t, $J=7.4 \mathrm{~Hz}, 2 \mathrm{H}), 7.51-7.47(\mathrm{~m}, 1 \mathrm{H}), 7.43-7.38$ (m, 2H), 7.29-7.24 (m, 4H), 7.22 (s, 1H), 7.20-7.17 (m, 1H); ${ }^{13} \mathrm{C}$ NMR (100 MHz, $\left.\mathrm{CDCl}_{3}\right): \delta$ 194.9, 146.5, 145.6, 145.2, 144.5, 144.1, 139.9, 139.1, 134.9, 133.8, 129.6, 128.9, 128.7, 128.6, 128.1, 127.8, 127.7, 126.9, 126.2, 125.0, 124.6, 122.7, 121.9, 74.1; HRMS (ES $\left.{ }^{+}-\mathrm{TOF}\right)$ calcd for $\mathrm{C}_{28} \mathrm{H}_{19} \mathrm{O}\left([\mathrm{M}+\mathrm{H}]^{+}\right)$: 371.1430, found 371.1433.

\section{8-methyl-4b,10-diphenylindeno[2,1-a]inden-5(4bH)-one (2b)}

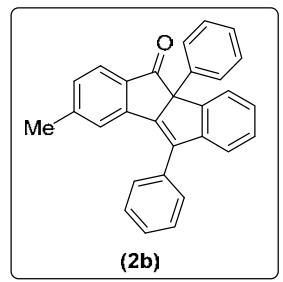

The reaction of 2,4,4-triphenyl-1-(p-tolyl)buta-2,3-dien-1-one $\mathbf{1 b}$ (77.2 $\mathrm{mg}, 0.2$ mmol, 1.0 equiv), $\mathrm{FeCl}_{3}$ (65 mg, $0.4 \mathrm{mmol}, 2.0$ equiv), in $2 \mathrm{~mL}$ of $\mathrm{DCM}$ at $50{ }^{\circ} \mathrm{C}$ for $12 \mathrm{~h}$ afforded $\mathbf{2 b}$ (72.6 mg, 94\%) as a yellow solid; m.p. 194-196 ${ }^{\circ} \mathrm{C}$ 
(Petroleum ether/EtOAc); R $f=0.45$ (Petroleum ether/EtOAc $=10 / 1) ;{ }^{1} \mathrm{H}$ NMR $\left(400 \mathrm{MHz}, \mathrm{CDCl}_{3}\right): \delta$ 7.76-7.71 (m, 3H), 7.68-7.63 (m, 3H), 7.58 (t, $J=7.6 \mathrm{~Hz}, 2 \mathrm{H}), 7.51-7.49$ (m, 1H), 7.43 (s, 1H), 7.38-7.37 (m, 1H), 7.26-7.22 (m, 4H), 7.19-7.18 (m, 1H), 7.04 (d, $J=8.0 \mathrm{~Hz}, 1 \mathrm{H}), 2.29(\mathrm{~s}, 3 \mathrm{H}) ;{ }^{13} \mathrm{C}$ NMR (100 MHz, $\left.\mathrm{CDCl}_{3}\right): \delta 194.4,146.6,146.0,145.6,145.3,144.4,142.3,140.2,138.7,134.0$, 129.6, 129.3, 128.9, 128.7, 128.5, 127.7, 127.5, 126.8, 126.2, 124.8, 124.6, 123.2, 121.8, 74.4, 22.2; HRMS (ES ${ }^{+}$-TOF) calcd for $\mathrm{C}_{29} \mathrm{H}_{21} \mathrm{O}\left([\mathrm{M}+\mathrm{H}]^{+}\right)$: 385.1587, found 385.1595.

\section{8-methoxy-4b,10-diphenylindeno[2,1-a]inden-5(4bH)-one (2c)}

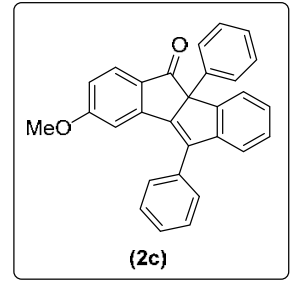

The reaction of 1-(4-methoxyphenyl)-2,4,4-triphenylbuta-2,3-dien-1-one 1c (80.9 mg, $0.2 \mathrm{mmol}, 1.0$ equiv), $\mathrm{FeCl}_{3}$ (65 mg, $0.4 \mathrm{mmol}, 2.0$ equiv), in $2 \mathrm{~mL}$ of DCM at $50{ }^{\circ} \mathrm{C}$ for $12 \mathrm{~h}$ afforded 2c (75.0 mg, 92\%) as a yellow solid; m.p. 197-198 ${ }^{\circ} \mathrm{C}$

(Petroleum ether/EtOAc); R $f=0.26($ Petroleum ether/EtOAc $=10 / 1) ;{ }^{1} \mathrm{H}$ NMR $\left(400 \mathrm{MHz}, \mathrm{CDCl}_{3}\right): \delta$ 7.76-7.74 (m, 1H), 7.72-7.67 (m, 5H), 7.56 (t, $J=7.2 \mathrm{~Hz}, 2 \mathrm{H})$, 7.50-7.46 (m, 1H), 7.39-7.37 (m, 1H), 7.28-7.22 (m, 4H), 7.20-7.16 (m, 1H), 7.08 (d, $J=2.0 \mathrm{~Hz}, 1 \mathrm{H}), 6.75\left(\mathrm{dd}, J_{1}=4.4 \mathrm{~Hz}, J_{2}=1.0 \mathrm{~Hz}\right.$, 1H), 3.72 (s, 3H); ${ }^{13} \mathrm{C}$ NMR (100 MHz, $\left.\mathrm{CDCl}_{3}\right): \delta 193.2,165.1,146.5,146.4,145.5,145.4,140.3$, 139.2, 137.6, 133.9, 129.6, 128.85, 128.76, 128.5, 127.7, 127.5, 127.0, 126.7, 126.2, 124.7, 121.9, 114.8, 107.0, 74.2, 55.5; HRMS (ES $-\mathrm{TOF})$ calcd for $\mathrm{C}_{29} \mathrm{H}_{21} \mathrm{O}_{2}\left([\mathrm{M}+\mathrm{H}]^{+}\right)$: 401.1536, found 401.1546.

\section{7,9-dimethoxy-4b,10-diphenylindeno[2,1-a]inden-5(4bH)-one (2d)}

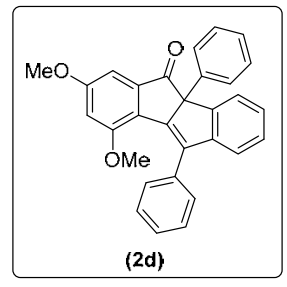

The reaction of 1-(3,5-dimethoxyphenyl)-2,4,4-triphenylbuta-2,3-dien-1-one 1d (86.5 mg, $0.2 \mathrm{mmol}, 1.0$ equiv), $\mathrm{FeCl}_{3}$ (65 mg, $0.4 \mathrm{mmol}, 2.0$ equiv), in $2 \mathrm{~mL}$ of DCM at $50{ }^{\circ} \mathrm{C}$ for $12 \mathrm{~h}$ afforded $2 \mathbf{d}$ (40.8 mg, 47\%) as a yellow solid; m.p.

156-158 ${ }^{\circ} \mathrm{C}$ (Petroleum ether/EtOAc); R $f=0.30$ (Petroleum ether/EtOAc $\left.=5 / 1\right) ;{ }^{1} \mathrm{H}$ NMR $(400 \mathrm{MHz}$, 
$\left.\mathrm{CDCl}_{3}\right): \delta$ 7.74-7.71 (m, 1H), 7.68-7.66 (m , 2H), 7.48-7.38 (m, 5H), 7.23-7.14 (m, 6H), $6.87(\mathrm{~d}, J=$ $1.0 \mathrm{~Hz}, 1 \mathrm{H}), 6.55$ (d, $J=1.0 \mathrm{~Hz}, 1 \mathrm{H}), 3.79$ (s, 3H), 3.44 (s, 3H); ${ }^{13} \mathrm{C}$ NMR $\left(100 \mathrm{MHz}, \mathrm{CDCl}_{3}\right): \delta$ 194.5, 161.4, 156.3, 147.4, 147.1, 144.0, 143.8, 140.4, 135.4, 134.8, 129.1, 128.9, 127.9, 127.7, 127.6, 127.4, 126.2, 125.9, 124.0, 121.5, 106.1, 99.2, 75.3, 55.8, 55.0; HRMS (ES ${ }^{+}$-TOF) calcd for $\mathrm{C}_{30} \mathrm{H}_{23} \mathrm{O}_{3}\left([\mathrm{M}+\mathrm{H}]^{+}\right):$431.1642, found 431.1646.

\section{8-chloro-4b,10-diphenylindeno[2,1-a]inden-5(4bH)-one (2e)}

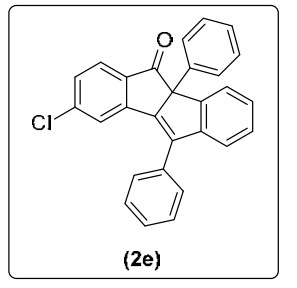

The reaction of 1-(4-chlorophenyl)-2,4,4-triphenylbuta-2,3-dien-1-one 1e (81.4 mg, $0.2 \mathrm{mmol}, 1.0$ equiv), $\mathrm{FeCl}_{3}$ (65 mg, $0.4 \mathrm{mmol}, 2.0$ equiv), in $2 \mathrm{~mL}$ of DCM at $50{ }^{\circ} \mathrm{C}$ for $12 \mathrm{~h}$ afforded $2 \mathrm{e}$ (73.0 mg, 89\%) as a yellow solid; m.p. $188-189{ }^{\circ} \mathrm{C}$ (Petroleum ether/EtOAc); R $f=0.45$ (Petroleum ether/EtOAc $=10 / 1) ;{ }^{1} \mathrm{H}$ NMR $\left(400 \mathrm{MHz}, \mathrm{CDCl}_{3}\right): \delta$ 7.75-7.73 (m, 1H), 7.70-7.68 (m, 2H), 7.67-7.64 (m, 3H), 7.61-7.57 (m, 3H), 7.53-7.50 (m, 1H), 7.40-7.38 (m, 1H), 7.29-7.23 (m, 4H), 7.21-7.18 (m, 2H); ${ }^{13} \mathrm{C}$ NMR (100 MHz, $\left.\mathrm{CDCl}_{3}\right): \delta 193.6$, 145.3, 144.8, 142.8, 141.4, 140.6, 139.5, 133.4, 129.5, 129.1, 129.0, 128.8, 128.4, 127.9, 127.8, 127.3, 126.1, 126.0, 124.7, 122.8, 122.2, 74.4; HRMS (ES ${ }^{+}$TOF) calcd for $\mathrm{C}_{28} \mathrm{H}_{18} \mathrm{ClO}\left([\mathrm{M}+\mathrm{H}]^{+}\right)$: 405.1041, found 405.1049.

\section{7-chloro-4b,10-diphenylindeno[2,1-a]inden-5(4bH)-one (2f)}

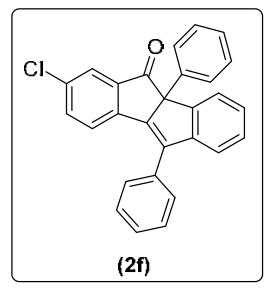

The reaction of 1-(3-chlorophenyl)-2,4,4-triphenylbuta-2,3-dien-1-one $\mathbf{1 f}$ (81.4 mg, $0.2 \mathrm{mmol}, 1.0$ equiv), $\mathrm{FeCl}_{3}$ (65 mg, $0.4 \mathrm{mmol}, 2.0$ equiv), in $2 \mathrm{~mL}$ of DCM at 50 ${ }^{\circ} \mathrm{C}$ for $12 \mathrm{~h}$ afforded $2 \mathrm{f}$ (71.2 mg, 87\%) as a yellow solid; m.p. 140-142 ${ }^{\circ} \mathrm{C}$ (Petroleum ether/EtOAc); R $f=0.45$ (Petroleum ether/EtOAc $=10 / 1) ;{ }^{1} \mathrm{H}$ NMR $\left(400 \mathrm{MHz}, \mathrm{CDCl}_{3}\right): \delta$ 7.75-7.73 (m, 1H), 7.71-7.68 (m, 3H), 7.66-7.64 (m, 2H), 7.59-7.55 (m, 3H), 7.52-7.50 (m, 1H), 7.40-7.34 (m. 2H), 7.29-7.20 (m, 5H); $\left.{ }^{13} \mathrm{C} \mathrm{NMR} \mathrm{(100} \mathrm{MHz,} \mathrm{CDCl}_{3}\right): \delta$ 193.7, 145.7, 145.4, 145.1, 
145.0, 142.2, 139.8, 139.3, 134.8, 134.5, 133.6, 129.5, 129.1, 128.9, 128.7, 127.91, 127.87, 127.2, 126.2, 125.0, 124.6, 123.6, 122.1, 74.5; HRMS (ES $\left.{ }^{+}-\mathrm{TOF}\right)$ calcd for $\mathrm{C}_{28} \mathrm{H}_{18} \mathrm{ClO}\left([\mathrm{M}+\mathrm{H}]^{+}\right)$: 405.1041, found 405.1042 .

\section{8-bromo-4b,10-diphenylindeno[2,1-a]inden-5(4bH)-one (2g)}

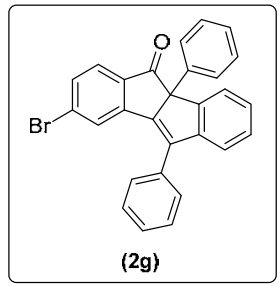

The reaction of 1-(4-bromophenyl)-2,4,4-triphenylbuta-2,3-dien-1-one $\mathbf{1 g}$ (90.2 mg, $0.2 \mathrm{mmol}, 1.0$ equiv), $\mathrm{FeCl}_{3}$ (65 mg, $0.4 \mathrm{mmol}, 2.0$ equiv), in $2 \mathrm{~mL}$ of DCM at $50{ }^{\circ} \mathrm{C}$ for $12 \mathrm{~h}$ afforded $\mathbf{2 g}$ (80.5 mg, 93\%) as a yellow solid; m.p. $213-215^{\circ} \mathrm{C}$

(Petroleum ether/EtOAc); $\mathrm{R} f=0.45$ (Petroleum ether/EtOAc $=10 / 1) ;{ }^{1} \mathrm{H}$ NMR $\left(400 \mathrm{MHz}, \mathrm{CDCl}_{3}\right): \delta$ 7.76-7.73 (m, 2H), 7.70-7.68 (m, 2H), 7.65-7.58 (m, 5H), 7.54-7.50 (m, 1H), 7.40-7.36 (m, 2H), 7.30-7.26 (m, 3H), 7.23-7.20 (m, 2H) ; $\left.{ }^{13} \mathrm{C} \mathrm{NMR} \mathrm{(100} \mathrm{MHz,} \mathrm{CDCl}_{3}\right): \delta$ 193.8, 145.4, 145.2, 144.7, 143.2, 140.6, 139.4, 133.4, 131.3, 130.2, 129.5, 129.1, 129.0, 128.8, 127.9, 127.8, 127.3, 126.14, 126.10, 125.8, 124.7, 122.3, 74.3; HRMS (ES ${ }^{+}$TOF) calcd for $\mathrm{C}_{28} \mathrm{H}_{18} \mathrm{BrO}\left([\mathrm{M}+\mathrm{H}]^{+}\right)$: 449.0536, found 449.0544 .

\section{4b,10-diphenyl-8-(trifluoromethyl)indeno[2,1-a]inden-5(4bH)-one (2h)}

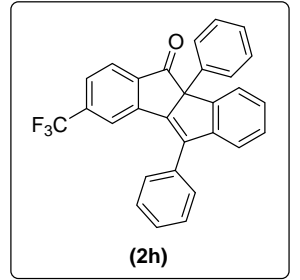

The reaction of 2,4,4-triphenyl-1-(4-(trifluoromethyl)phenyl)buta-2,3-dien-1-one 1h (88.1 mg, 0.2 mmol, 1.0 equiv), $\mathrm{FeCl}_{3}$ (65 mg, $0.4 \mathrm{mmol}, 2.0$ equiv), in $2 \mathrm{~mL}$ of DCM at $50{ }^{\circ} \mathrm{C}$ for $12 \mathrm{~h}$ afforded $2 \mathrm{~g}(74.0 \mathrm{mg}, 83 \%)$ as a yellow solid; m.p. 214-216 ${ }^{\circ} \mathrm{C}$ (Petroleum ether/EtOAc); $\mathrm{R} f=0.40$ (Petroleum ether/EtOAc $\left.=10 / 1\right) ;{ }^{1} \mathrm{H}$ NMR $(400$ $\left.\mathrm{MHz}, \mathrm{CDCl}_{3}\right): \delta$ 7.87-7.82 (m, 2H), 7.76-7.71 (m, 3H), $7.65(\mathrm{~d}, J=8.0 \mathrm{~Hz}, 2 \mathrm{H}), 7.60(\mathrm{t}, J=7.4 \mathrm{~Hz}$, 2H), 7.55-7.48 (m, 2H), 7.44-7.42 (m, 1H), 7.32-7.21 (m, 5H); ${ }^{13} \mathrm{C}$ NMR (100 MHz, $\left.\mathrm{CDCl}_{3}\right): \delta$ 194.0, 146.9, 145.2, 144.6, 144.2, 141.1, 139.0, $136.0\left(\mathrm{q},{ }^{2} J_{C-F}=32.0 \mathrm{~Hz}\right), 133.2,129.4,129.3,129.1,128.8$, 128.0, 127.5, 126.2, 125.4, $124.9\left(\mathrm{q},{ }^{3} J_{C-F}=1.7 \mathrm{~Hz}\right), 124.7,123.3\left(\mathrm{q},{ }^{1} J_{C-F}=275.0 \mathrm{~Hz}\right), 122.4,119.5$ 
(broad), 74.6; HRMS (ES ${ }^{+}$-TOF) calcd for $\mathrm{C}_{29} \mathrm{H}_{18} \mathrm{~F}_{3} \mathrm{O}\left([\mathrm{M}+\mathrm{H}]^{+}\right)$: 439.1304, found 439.1311.

\section{4,8b-diphenylbenzo[4,5]pentaleno[2,1-b]thiophen-9(8bH)-one (2i)}

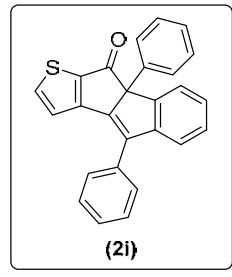

The reaction of 2,4,4-triphenyl-1-(thiophen-2-yl)buta-2,3-dien-1-one 1i (75.7 mg, 0.2 mmol, 1.0 equiv), $\mathrm{FeCl}_{3}$ (65 mg, $0.4 \mathrm{mmol}, 2.0$ equiv), in $2 \mathrm{~mL}$ of $\mathrm{DCM}$ at $50{ }^{\circ} \mathrm{C}$ for $12 \mathrm{~h}$ afforded $2 \mathbf{i}$ (43.1 mg, 57\%) as a yellow solid; m.p. 174-176 ${ }^{\circ} \mathrm{C}$ (Petroleum ether/EtOAc); $\mathrm{R} f=0.50$ (Petroleum ether/EtOAc $=10 / 1) ;{ }^{1} \mathrm{H}$ NMR $\left(400 \mathrm{MHz}, \mathrm{CDCl}_{3}\right): \delta 7.79-7.62$ (m, 6H), 7.59-7.41 (m, 4H), 7.32-7.15 (m, 5H), $7.08(\mathrm{~d}, J=4.0 \mathrm{~Hz}, 1 \mathrm{H}) ;{ }^{13} \mathrm{C}$ NMR $(100 \mathrm{MHz}$, $\left.\mathrm{CDCl}_{3}\right): \delta 186.5,157.1,146.5,145.4,144.7,143.3,140.8,140.4,135.7,133.8,129.0,128.83,128.78$, 128.7, 127.8, 127.6, 127.2, 126.1, 124.6, 122.1, 121.2, 77.9; HRMS (ES $\left.{ }^{+}-\mathrm{TOF}\right)$ calcd for $\mathrm{C}_{26} \mathrm{H}_{17} \mathrm{OS}$ $\left([\mathrm{M}+\mathrm{H}]^{+}\right):$377.0995, found 377.0998.

\section{7a,12-diphenylbenzo[4,5]pentaleno[1,2-a]naphthalen-7(7aH)-one (2j)}

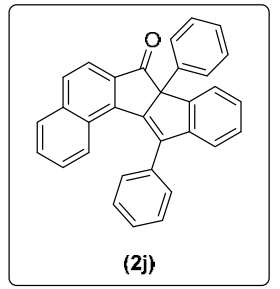

The reaction of 1-(naphthalen-2-yl)-2,4,4-triphenylbuta-2,3-dien-1-one 1j (84.6 mg, $0.2 \mathrm{mmol}, 1.0$ equiv), $\mathrm{FeCl}_{3}$ (65 mg, $0.4 \mathrm{mmol}, 2.0$ equiv), in $2 \mathrm{~mL}$ of $\mathrm{DCM}$ at 50 ${ }^{\circ} \mathrm{C}$ for $12 \mathrm{~h}$ afforded $\mathbf{2 j}$ (60.6 mg, 72\%) as a yellow solid; m.p. $238-240{ }^{\circ} \mathrm{C}$ (Petroleum ether/EtOAc); R $f=0.50$ (Petroleum ether/EtOAc $=10 / 1) ;{ }^{1} \mathrm{H}$ NMR $\left(400 \mathrm{MHz}, \mathrm{CDCl}_{3}\right): \delta$ 7.84-7.79 (m, 3H), 7.75-7.70 (m, 3H), 7.51-7.45 (m, 2H), 7.40-7.34 (m, 5H), 7.23-7.14 (m, 7H); ${ }^{13} \mathrm{C}$ NMR (100 MHz, $\left.\mathrm{CDCl}_{3}\right): \delta 193.7,146.9,145.6,145.5,144.6,143.4,140.3,137.1,136.7,134.5$, 129.04, 128.98, 128.9, 128.5, 128.2, 127.9, 127.6, 127.5, 126.8, 126.5, 126.1, 124.4, 122.0, 120.5, 76.2; HRMS (ES ${ }^{+}$-TOF) calcd for $\mathrm{C}_{32} \mathrm{H}_{21} \mathrm{O}\left([\mathrm{M}+\mathrm{H}]^{+}\right)$: 421.1587, found 421.1591.

\section{4b-(4-methoxyphenyl)-10-phenylindeno[2,1-a]inden-5(4bH)-one (2k)}

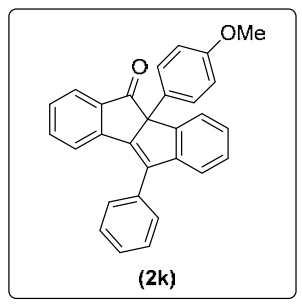

The reaction of 2-(4-methoxyphenyl)-1,4,4-triphenylbuta-2,3-dien-1-one $\mathbf{1 k}$ (80.4 mg, $0.2 \mathrm{mmol}, 1.0$ equiv), $\mathrm{FeCl}_{3}$ (65 mg, $0.4 \mathrm{mmol}, 2.0$ equiv), in $2 \mathrm{~mL}$ of 
DCM at $50{ }^{\circ} \mathrm{C}$ for $12 \mathrm{~h}$ afforded $2 \mathbf{k}$ (75.6 mg, 94\%) as a yellow solid; m.p. 151-153 ${ }^{\circ} \mathrm{C}$ (Petroleum ether/EtOAc); $\mathrm{R} f=0.25$ (Petroleum ether/EtOAc = 10/1); ${ }^{1} \mathrm{H}$ NMR $\left(400 \mathrm{MHz}, \mathrm{CDCl}_{3}\right): \delta$ 7.77-7.66 (m, 4H), 7.65-7.51 (m, 5H), 7.46 (t, $J=7.2 \mathrm{~Hz}, 1 \mathrm{H}), 7.40-7.32$ (m, 2H), 7.27-7.16 (m, 3H), 6.76 (d, $J$ = 8.8 Hz, 2H), 3.65 (s, 3H); ${ }^{13} \mathrm{C}$ NMR (100 MHz, $\left.\mathrm{CDCl}_{3}\right): \delta$ 194.9, 159.0, 146.6, 145.5, 145.4, 144.4, 143.9, 138.9, 134.7, 133.8, 131.8, 129.5, 128.6, 128.5, 128.1, 127.6, 127.3, 126.8, 124.9, 124.5, 122.6, 121.8, 114.3, 73.4, 55.1; HRMS (ES ${ }^{+}$-TOF) calcd for $\mathrm{C}_{29} \mathrm{H}_{21} \mathrm{O}_{2}\left([\mathrm{M}+\mathrm{H}]^{+}\right)$: 401.1536, found 401.1540.

\section{4b-(4-chlorophenyl)-10-phenylindeno[2,1-a]inden-5(4bH)-one (2l)}

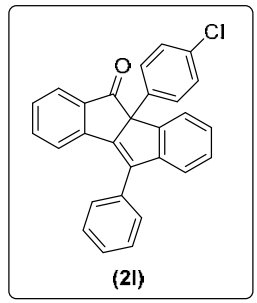

The reaction of 2-(4-chlorophenyl)-1,4,4-triphenylbuta-2,3-dien-1-one $\mathbf{1 l}$ (81.4 mg, $0.2 \mathrm{mmol}, 1.0$ equiv), $\mathrm{FeCl}_{3}$ (65 mg, $0.4 \mathrm{mmol}, 2.0$ equiv), in $2 \mathrm{~mL}$ of DCM at 50 ${ }^{\circ} \mathrm{C}$ for $12 \mathrm{~h}$ afforded $2 \mathbf{l}$ (62.7 mg, 77\%) as a yellow solid; m.p. 169-171 ${ }^{\circ} \mathrm{C}$ (Petroleum ether/EtOAc); $\mathrm{R} f=0.40$ (Petroleum ether/EtOAc $=10 / 1) ;{ }^{1} \mathrm{H}$ NMR $\left(400 \mathrm{MHz}, \mathrm{CDCl}_{3}\right): \delta$ 7.76-7.69 (m, 4H), 7.64-7.55 (m, 5H), 7.52-7.48 (m, 1H), 7.45-7.38 (m, 2H), 7.31-7.27 (m, 3H), 7.21 (d, $J=8.4 \mathrm{~Hz}, 2 \mathrm{H}) ;{ }^{13} \mathrm{C}$ NMR $\left(100 \mathrm{MHz}, \mathrm{CDCl}_{3}\right): \delta 194.6,146.1,145.5,144.9,144.3,144.0,139.5$, 138.4, 135.1, 133.6, 129.6, 129.1, 128.85, 128.77, 128.6, 128.3, 128.0, 127.6, 127.1, 125.1, 124.6, 122.7, 122.1, 73.5; HRMS (ES ${ }^{+}$-TOF) calcd for $\mathrm{C}_{28} \mathrm{H}_{18} \mathrm{ClO}\left([\mathrm{M}+\mathrm{H}]^{+}\right)$: 405.1041, found 405.1038.

\section{4b-(4-bromophenyl)-10-phenylindeno[2,1-a]inden-5(4bH)-one (2m)}

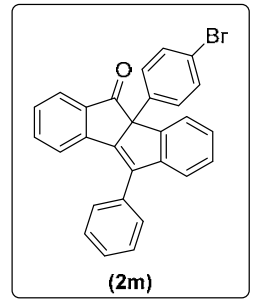

The reaction of 2-(4-bromophenyl)-1,4,4-triphenylbuta-2,3-dien-1-one 1m (90.2 mg, $0.2 \mathrm{mmol}, 1.0$ equiv), $\mathrm{FeCl}_{3}$ (65 mg, $0.4 \mathrm{mmol}, 2.0$ equiv), in $2 \mathrm{~mL}$ of $\mathrm{DCM}$ at $50{ }^{\circ} \mathrm{C}$ for $12 \mathrm{~h}$ afforded $\mathbf{2 m}$ (82.0 mg, 91\%) a yellow solid; m.p. 188-190 ${ }^{\circ} \mathrm{C}$ (Petroleum ether/EtOAc); R $f=0.40$ (Petroleum ether/EtOAc $=10 / 1) ;{ }^{1} \mathrm{H}$ NMR $\left(400 \mathrm{MHz}, \mathrm{CDCl}_{3}\right): \delta 7.75(\mathrm{~d}, J$ $=7.8 \mathrm{~Hz}, 1 \mathrm{H}), 7.72-7.69(\mathrm{~m}, 3 \mathrm{H}), 7.63(\mathrm{~d}, J=13.6 \mathrm{~Hz}, 1 \mathrm{H}), 7.69-7.48(\mathrm{~m}, 5 \mathrm{H}), 7.45-7.35(\mathrm{~m}, 4 \mathrm{H})$, 
7.31-7.25 (m, 3H); ${ }^{13} \mathrm{C}$ NMR (100 MHz, $\left.\mathrm{CDCl}_{3}\right): \delta$ 194.5, 146.0, 145.5, 144.8, 144.3, 143.9, 139.5, 139.0, 135.1, 133.6, 132.0, 129.5, 128.9, 128.6, 128.3, 128.0, 127.1, 125.1, 124.5, 122.7, 122.1, 121.8, 73.5; HRMS (ES ${ }^{+}$TOF) calcd for $\mathrm{C}_{28} \mathrm{H}_{18} \mathrm{BrO}\left([\mathrm{M}+\mathrm{H}]^{+}\right)$: 449.0536, found 449.0539.

\section{4b-(3-fluorophenyl)-10-phenylindeno[2,1-a]inden-5(4bH)-one (2n)}

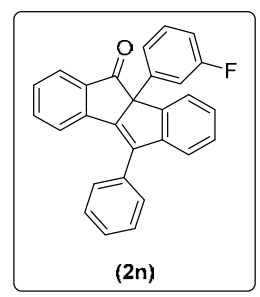

The reaction of 2-(3-fluorophenyl)-1,4,4-triphenylbuta-2,3-dien-1-one 1n (78.0 mg, $0.2 \mathrm{mmol}, 1.0$ equiv), $\mathrm{FeCl}_{3}$ (65 mg, $0.4 \mathrm{mmol}, 2.0$ equiv), in $2 \mathrm{~mL}$ of DCM at 50 ${ }^{\circ} \mathrm{C}$ for $12 \mathrm{~h}$ afforded 2n (45.8 mg, 58\%) as a yellow solid; m.p. $152-154{ }^{\circ} \mathrm{C}$ (Petroleum ether/EtOAc); R $f=0.40$ (Petroleum ether/EtOAc $=10 / 1) ;{ }^{1} \mathrm{H}$ NMR $\left(400 \mathrm{MHz}, \mathrm{CDCl}_{3}\right): \delta$ 7.77-7.70 (m, 4H), 7.64 (d, $J=7.8 \mathrm{~Hz}, 1 \mathrm{H}), 7.58$ (t, $J=7.8 \mathrm{~Hz}, 2 \mathrm{H}), 7.52-7.47$ (m, 2H), 7.45-7.35 (m, 3H), 7.31-7.25 (m, 3H), 7.23-7.21 (m, 1H), 6.90-6.88 (m, 1H); ${ }^{13} \mathrm{C}$ NMR (100 MHz, $\left.\mathrm{CDCl}_{3}\right): \delta$ 194.5, $162.9\left(\mathrm{~d},{ }^{1} J_{\mathrm{C}-\mathrm{F}}=245.2 \mathrm{~Hz}\right), 146.0,145.6,144.7,144.3,144.1,142.2\left(\mathrm{~d},{ }^{3} J_{\mathrm{C}-\mathrm{F}}=7.4 \mathrm{~Hz}\right), 139.6,135.1$, 133.6, $130.3\left(\mathrm{~d},{ }^{3} J_{\mathrm{C}-\mathrm{F}}=8.1 \mathrm{~Hz}\right), 129.6 .128 .9,128.6,128.3,128.0,127.1,125.1,124.6,122.8,122.1$, 122.0, $114.7\left(\mathrm{~d},{ }^{2} J_{\mathrm{C}-\mathrm{F}}=21.1 \mathrm{~Hz}\right), 113.4\left(\mathrm{~d},{ }^{2} J_{\mathrm{C}-\mathrm{F}}=22.9 \mathrm{~Hz}\right), 73.7$; HRMS (ES $\left.{ }^{+}-\mathrm{TOF}\right)$ calcd for $\mathrm{C}_{28} \mathrm{H}_{18} \mathrm{FO}\left([\mathrm{M}+\mathrm{H}]^{+}\right):$389.1336, found 389.1340.

\section{4b-methyl-10-phenylindeno[2,1-a]inden-5(4bH)-one (2o)}

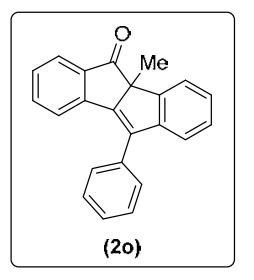

The reaction of 2-methyl-1,4,4-triphenylbuta-2,3-dien-1-one 10 (62.1 mg, 0.2 mmol, 1.0 equiv), $\mathrm{FeCl}_{3}$ (65 mg, $0.4 \mathrm{mmol}, 2.0$ equiv), in $2 \mathrm{~mL}$ of $\mathrm{DCM}$ at $50{ }^{\circ} \mathrm{C}$ for $12 \mathrm{~h}$ afforded 20 (46.8 mg, 75\%) as a yellow solid; m.p. 112-114 ${ }^{\circ} \mathrm{C}$ (Petroleum ether/EtOAc); $\mathrm{R} f=0.45($ Petroleum ether/EtOAc $=10 / 1) ;{ }^{1} \mathrm{H}$ NMR $\left(400 \mathrm{MHz}, \mathrm{CDCl}_{3}\right): \delta 7.81(\mathrm{~d}, J$ $=7.8 \mathrm{~Hz}, 1 \mathrm{H}), 7.78-7.76(\mathrm{~m}, 1 \mathrm{H}), 7.64-7.60(\mathrm{~m}, 3 \mathrm{H}), 7.53(\mathrm{t}, J=7.4 \mathrm{~Hz}, 2 \mathrm{H}), 7.47-7.44(\mathrm{~m}, 2 \mathrm{H})$, 7.42-7.40 (m, 1H), 7.34-7.32 (m, 3H), 1.72 (s, 3H); ${ }^{13} \mathrm{C}$ NMR (100 MHz, $\left.\mathrm{CDCl}_{3}\right): \delta$ 197.8, 149.1, 145.40, 145.37, 143.6, 143.4, 136.9, 134.8, 134.1, 129.5, 128.5, 128.0, 127.7, 126.7, 125.1, 123.7, 
122.9, 121.9, 66.5, 26.9; HRMS (ES ${ }^{+}$-TOF) calcd for $\mathrm{C}_{23} \mathrm{H}_{17} \mathrm{O}\left([\mathrm{M}+\mathrm{H}]^{+}\right)$: 309.1274, found 309.1279.

\section{4b-(4-bromophenyl)-8-methyl-10-phenylindeno[2,1-a]inden-5(4bH)-one (2p)}

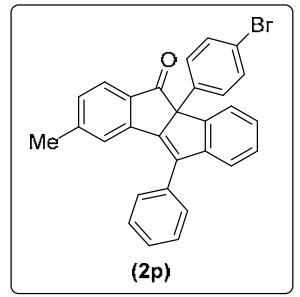

The reaction of 2-(4-bromophenyl)-4,4-diphenyl-1-(p-tolyl)buta-2,3-dien-1-one 1p (93.1 mg, 0.2 mmol, 1.0 equiv), $\mathrm{FeCl}_{3}$ (65 mg, $0.4 \mathrm{mmol}, 2.0$ equiv), in $2 \mathrm{~mL}$ of DCM at $50{ }^{\circ} \mathrm{C}$ for $12 \mathrm{~h}$ afforded 2p (82.2 mg, 88\%) as a yellow solid; m.p.

193-195 ${ }^{\circ} \mathrm{C}$ (Petroleum ether/EtOAc); $\mathrm{R} f=0.40$ (Petroleum ether/EtOAc $\left.=10 / 1\right) ;{ }^{1} \mathrm{H}$ NMR $(400$ $\left.\mathrm{MHz}, \mathrm{CDCl}_{3}\right): \delta$ 7.71-7.65 (m, 3H), $7.64(\mathrm{~d}, J=7.6 \mathrm{~Hz}, 1 \mathrm{H}), 7.59-7.50(\mathrm{~m}, 5 \mathrm{H}), 7.42(\mathrm{~s}, 1 \mathrm{H})$, 7.39-7.34 (m, 3H), 7.28-7.24 (m, 2H), 7.08-7.06 (d, $J=8.0 \mathrm{~Hz}, 1 \mathrm{H}), 2.30(\mathrm{~s}, 3 \mathrm{H}) ;{ }^{13} \mathrm{C}$ NMR $(100$ $\left.\mathrm{MHz}, \mathrm{CDCl}_{3}\right): \delta 194.1,146.3,146.1,145.5,144.8,144.2,142.0,139.3,139.0,133.7,132.0,129.6$, 129.5, 128.8, 128.6, 127.9, 127.0, 124.9, 124.5, 123.2, 122.0, 121.6, 73.7, 22.3; HRMS (ES ${ }^{+}$-TOF) calcd for $\mathrm{C}_{29} \mathrm{H}_{20} \mathrm{BrO}\left([\mathrm{M}+\mathrm{H}]^{+}\right)$: 463.0692, found 463.0693 .

\section{3-methoxy-10-(4-methoxyphenyl)-4b-phenylindeno[2,1-a]inden-5(4bH)-one (2q)}

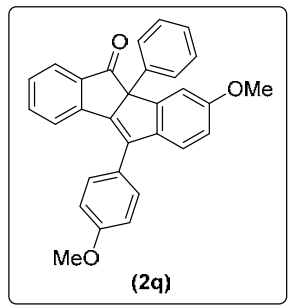

The reaction of 4,4-bis(4-methoxyphenyl)-1,2-diphenylbuta-2,3-dien-1-one 1q (86.6 mg, $0.2 \mathrm{mmol}, 1.0$ equiv), $\mathrm{FeCl}_{3}$ (65 mg, $0.4 \mathrm{mmol}, 2.0$ equiv), in $2 \mathrm{~mL}$ of DCM at $50{ }^{\circ} \mathrm{C}$ for $12 \mathrm{~h}$ afforded $\mathbf{2 q}(19.8 \mathrm{mg}, 23 \%)$ as a yellow solid; m.p.

197-199 ${ }^{\circ} \mathrm{C}$ (Petroleum ether/EtOAc); R $f=0.30$ (Petroleum ether/EtOAc $\left.=5 / 1\right) ;{ }^{1} \mathrm{H}$ NMR $(400 \mathrm{MHz}$, $\left.\mathrm{CDCl}_{3}\right): \delta 7.73(\mathrm{~d}, J=7.8 \mathrm{~Hz}, 1 \mathrm{H}), 7.66-7.64(\mathrm{~m}, 4 \mathrm{H}), 7.61(\mathrm{~d}, J=7.6 \mathrm{~Hz}, 1 \mathrm{H}), 7.42-7.38(\mathrm{~m}, 1 \mathrm{H})$, 7.61-7.28 (m, 2H), 7.24-7.19 (m, 4H), $7.09(\mathrm{~d}, J=8.8 \mathrm{~Hz}, 2 \mathrm{H}), 6.80\left(\mathrm{dd}, J_{1}=8.4 \mathrm{~Hz}, J_{2}=2.4 \mathrm{~Hz}\right.$, 1H), 3.92 (s, 3H), 3.83 (s, 3H); ${ }^{13} \mathrm{C}$ NMR (100 MHz, $\left.\mathrm{CDCl}_{3}\right): \delta$ 195.4, 160.0, 159.4, 147.2, 144.8, 144.0, 143.8, 140.2, 139.0, 138.6, 124.9, 130.9, 128.9, 127.6, 127.5, 126.4, 126.2, 125.0, 122.4, 122.3, 114.0, 113.2, 111.2, 73.6, 55.6, 55.4; HRMS (ES ${ }^{+}$TOF) calcd for $\mathrm{C}_{30} \mathrm{H}_{23} \mathrm{O}_{3}\left([\mathrm{M}+\mathrm{H}]^{+}\right)$: 431.1642, found 431.1642. 


\section{3-chloro-10-(4-chlorophenyl)-4b-phenylindeno[2,1-a]inden-5(4bH)-one (2r)}

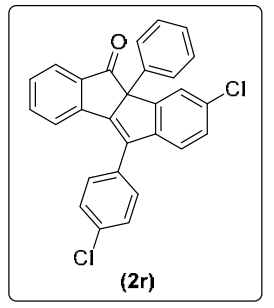

The reaction of 4,4-bis(4-chlorophenyl)-1,2-diphenylbuta-2,3-dien-1-one 1r (88.4 mg, $0.2 \mathrm{mmol}, 1.0$ equiv), $\mathrm{FeCl}_{3}$ (65 mg, $0.4 \mathrm{mmol}, 2.0$ equiv), in $2 \mathrm{~mL}$ of DCM at $50{ }^{\circ} \mathrm{C}$ for $12 \mathrm{~h}$ afforded 2r (82.8 mg, 95\%) as a yellow solid; m.p. 207-209 ${ }^{\circ} \mathrm{C}$ (Petroleum ether/EtOAc); Rf = 0.45 (Petroleum ether/EtOAc = 10/1); ${ }^{1} \mathrm{H}$ NMR $\left(400 \mathrm{MHz}, \mathrm{CDCl}_{3}\right): \delta$ 7.76 (d, $J=7.2 \mathrm{~Hz}, 1 \mathrm{H}), 7.73-7.70$ (m, 1H), 7.65-7.51 (m, 7H), 7.45 (t, $J=7.8 \mathrm{~Hz}, 1 \mathrm{H}), 7.32-7.20$ (m, $6 \mathrm{H}) ;{ }^{13} \mathrm{C}$ NMR (100 MHz, $\left.\mathrm{CDCl}_{3}\right): \delta$ 193.8, 147.2, 146.5, 144.4, 143.7, 143.5, 139.0, 137.0, 135.1, 134.8, 133.2, 131.9, 130.7, 129.1, 129.0, 128.6, 128.1, 128.0, 126.1, 125.3, 122.7, 122.3, 74.0; HRMS (ES ${ }^{+}$TOF) calcd for $\mathrm{C}_{28} \mathrm{H}_{17} \mathrm{Cl}_{2} \mathrm{O}\left([\mathrm{M}+\mathrm{H}]^{+}\right)$: 439.0651, found 439.0651 .

\section{9b-phenyl-6,9b-dihydrodibenzo[cd,h]indeno[1,2-a]azulen-10(5H)-one (2s)}

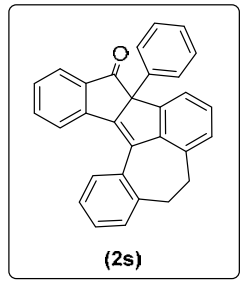

The reaction of 3-(10,11-dihydro-5H-dibenzo[a,d][7]annulen-5-ylidene)-1,2diphenylprop-2-en-1-one 1s (79.7 mg, 0.2 mmol, 1.0 equiv), $\mathrm{FeCl}_{3}$ (65 mg, 0.4 mmol, 2.0 equiv), in $2 \mathrm{~mL}$ of DCM at $50{ }^{\circ} \mathrm{C}$ for $12 \mathrm{~h}$ afforded $2 \mathrm{~s}(62.2 \mathrm{mg}, 78 \%)$ as a yellow solid; m.p. $205-206{ }^{\circ} \mathrm{C}$ (Petroleum ether/EtOAc); $\mathrm{R} f=0.40$ (Petroleum ether/EtOAc $=10 / 1$ ); ${ }^{1} \mathrm{H}$ NMR (400 MHz, $\mathrm{CDCl}_{3}$ ): $\delta 8.03$ (s, 2H), 7.79 (d, $\left.J=7.6 \mathrm{~Hz}, 1 \mathrm{H}\right)$, 7.64-7.58 (m, 3H), 7.50-7.36 (m, 3H), 7.30-7.24 (m, 2H), 7.21-7.17 (m, 3H), 7.10 (t, $J=7.6 \mathrm{~Hz}, 1 \mathrm{H}), 7.01$ (d, $J=7.6 \mathrm{~Hz}, 1 \mathrm{H})$, 3.12-2.97 (m, 4H); ${ }^{13} \mathrm{C}$ NMR (100 MHz, $\left.\mathrm{CDCl}_{3}\right): \delta 195.4,145.1,144.7,144.4,143.0,142.5,140.4$, 138.6, 134.8, 133.2, 133.0, 129.6, 129.3, 128.9, 128.6, 128.3, 127.5, 126.4, 126.2, 125.7, 125.1, 122.4, 122.2, 77.2, 36.3, 34.1; HRMS (ES $\left.{ }^{+}-\mathrm{TOF}\right)$ calcd for $\mathrm{C}_{30} \mathrm{H}_{21} \mathrm{O}\left([\mathrm{M}+\mathrm{H}]^{+}\right)$: 397.1587, found 397.1591.

8-bromo-3-methyl-4b-phenyl-10-(p-tolyl)indeno[2,1-a]inden-5(4bH)-one (2t) 


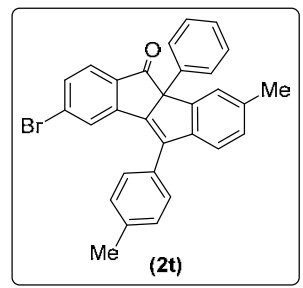

The reaction of 1-(4-bromophenyl)-2-phenyl-4,4-di-p-tolylbuta-2,3-dien-1-one $\mathbf{1 t}$ (95.9 mg, $0.2 \mathrm{mmol}, 1.0$ equiv), $\mathrm{FeCl}_{3}$ (65 mg, $0.4 \mathrm{mmol}, 2.0$ equiv), in $2 \mathrm{~mL}$ of DCM at $50{ }^{\circ} \mathrm{C}$ for $12 \mathrm{~h}$ afforded $2 \mathrm{t}$ (51.0 $\left.\mathrm{mg}, 55 \%\right)$ as a yellow solid; m.p.

232-234 ${ }^{\circ} \mathrm{C}$ (Petroleum ether/EtOAc); $\mathrm{R} f=0.35$ (Petroleum ether/EtOAc $\left.=10 / 1\right) ;{ }^{1} \mathrm{H}$ NMR $(400$ $\left.\mathrm{MHz}, \mathrm{CDCl}_{3}\right): \delta 7.77(\mathrm{~d}, J=1.6 \mathrm{~Hz}, 1 \mathrm{H}), 7.64-7.54(\mathrm{~m}, 5 \mathrm{H}), 7.39$ (d, $\left.J=7.6 \mathrm{~Hz}, 2 \mathrm{H}\right), 7.36-7.33$ (m, 1H), 7.29-7.20 (m, 5H), 7.08 (d, $J=8.0 \mathrm{~Hz}, 1 \mathrm{H}), 2.49$ (s, 3H), 2.37 (s, 3H); ${ }^{13} \mathrm{C}$ NMR (100 MHz, $\left.\mathrm{CDCl}_{3}\right): \delta 194.4,145.7,145.5,143.3,142.9,142.8,140.8,139.7,139.1,137.5,130.9,130.5,130.2$, 129.4, 129.0, 128.6, 127.7, 126.2, 126.0, 125.7, 125.5, 122.0, 74.0, 21.6, 21.5; HRMS (ES $\left.{ }^{+}-\mathrm{TOF}\right)$ calcd for $\mathrm{C}_{30} \mathrm{H}_{22} \mathrm{BrO}\left([\mathrm{M}+\mathrm{H}]^{+}\right)$: 477.0849, found 477.0859 .

\section{3-chloro-4b,10-bis(4-chlorophenyl)indeno[2,1-a]inden-5(4bH)-one (2u)}

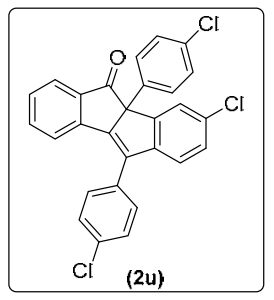

The reaction of 2,4,4-tris(4-chlorophenyl)-1-phenylbuta-2,3-dien-1-one 1u (95.2 mg, $0.2 \mathrm{mmol}, 1.0$ equiv), $\mathrm{FeCl}_{3}$ (65 mg, $0.4 \mathrm{mmol}$, 2.0 equiv), in $2 \mathrm{~mL}$ of DCM at $50{ }^{\circ} \mathrm{C}$ for $12 \mathrm{~h}$ afforded $2 \mathbf{u}$ (76.1 mg, 80\%) as a yellow solid; m.p. 243-245 ${ }^{\circ} \mathrm{C}$ (Petroleum ether/EtOAc); R $f=0.40$ (Petroleum ether/EtOAc $=10 / 1) ;{ }^{1} \mathrm{H}$ NMR $\left(400 \mathrm{MHz}, \mathrm{CDCl}_{3}\right): \delta$ 7.76 (d, $J=7.6 \mathrm{~Hz}, 1 \mathrm{H}), 7.68-7.67$ (m, 1H), 7.61-7.54 (m, 7H), 7.46 (t, $J=7.4 \mathrm{~Hz}, 1 \mathrm{H}), 7.32-7.27$ (m, 1H), 7.25-7.23 (m, 3H), 7.22-7.21 (m, 1H); ${ }^{13} \mathrm{C}$ NMR (100 MHz, $\left.\mathrm{CDCl}_{3}\right): \delta$ 193.5, 146.8, 146.2, 144.1, 143.6, 143.4, 137.5, 137.4, 135.3, 135.0, 134.0, 133.4, 131.6, 130.7, 129.3, 129.0, 128.7, 128.3, 127.5, 125.3, 125.2, 122.7, 122.5, 73.3; HRMS (ES ${ }^{+}$TOF) calcd for $\mathrm{C}_{28} \mathrm{H}_{16} \mathrm{Cl}{ }_{3} \mathrm{O}\left([\mathrm{M}+\mathrm{H}]^{+}\right)$: 473.0261, found 473.0276.

\section{2-methyl-4b,10-diphenylindeno[2,1-a]inden-5(4bH)-one (2v)}

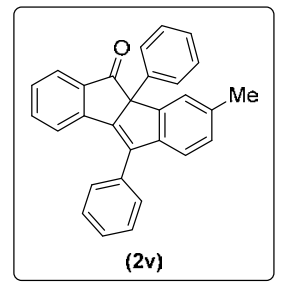

The reaction of 1,2,4-triphenyl-4-(p-tolyl)buta-2,3-dien-1-one $\mathbf{1 v}$ (77.2 mg, 0.2 mmol, 1.0 equiv), $\mathrm{FeCl}_{3}$ (65 mg, $0.4 \mathrm{mmol}, 2.0$ equiv), in $2 \mathrm{~mL}$ of DCM at $50{ }^{\circ} \mathrm{C}$ 
for $12 \mathrm{~h}$ afforded 2v (72.2 mg, 94\%) as a yellow solid; m.p. 164-166 ${ }^{\circ} \mathrm{C}$ (Petroleum ether/EtOAc); Rf $=0.45($ Petroleum ether $/$ EtOAc $=10 / 1) ;{ }^{1} \mathrm{H}$ NMR (400 MHz, $\left.\mathrm{CDCl}_{3}\right): \delta$ 7.75-7.67 (m, 5H), 7.63-7.54 (m, 4H), 7.50-7.48 (m, 1H), 7.41-7.37 (m, 2H), 7.28-7.25 (m, 2H), 7.23-7.19 (m, 2H), 7.07 (d, $J=$ 7.6 Hz, 1H), 2.37 (s, 3H); ${ }^{13} \mathrm{C}$ NMR (100 MHz, $\left.\mathrm{CDCl}_{3}\right): \delta$ 195.2, 145.7, 145.5, 144.4, 144.3, 143.0, 140.1, 139.2, 137.1, 134.8, 129.6, 129.5, 129.3, 128.9, 128.6, 128.5, 127.9, 127.6, 126.2, 125.6, 125.0, 122.6, 121.6, 74.0, 21.6; HRMS (ES ${ }^{+}$-TOF) calcd for $\mathrm{C}_{29} \mathrm{H}_{21} \mathrm{O}\left([\mathrm{M}+\mathrm{H}]^{+}\right)$: 385.1587, found 385.1594.

\section{0-(4-bromophenyl)-4b-phenylindeno[2,1-a]inden-5(4bH)-one (2w)}

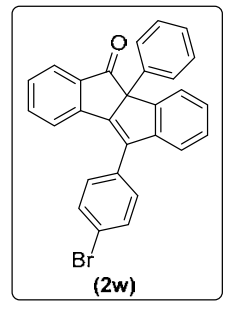

The reaction of 4-(4-bromophenyl)-1,2,4-triphenylbuta-2,3-dien-1-one $\mathbf{1 w}$ (90.3 mg, $0.2 \mathrm{mmol}, 1.0$ equiv), $\mathrm{FeCl}_{3}$ (65 mg, $0.4 \mathrm{mmol}, 2.0$ equiv), in $2 \mathrm{~mL}$ of $\mathrm{DCM}$ at $50{ }^{\circ} \mathrm{C}$ for $12 \mathrm{~h}$ afforded 2w (80.5 mg, 89\%) as a yellow solid; m.p. 211-213 ${ }^{\circ} \mathrm{C}$ (Petroleum ether/EtOAc); $\mathrm{R} f=0.45$ (Petroleum ether/EtOAc = 10/1); ${ }^{1} \mathrm{H}$ NMR $\left(400 \mathrm{MHz}, \mathrm{CDCl}_{3}\right): \delta$ 7.76-7.74 (m, 2H), 7.70-7.64 (m, 4H), 7.59-7.56 (m, 3H), 7.43-7.39 (m, 1H), 7.32-7.30 (m, 1H), 7.28-7.25 (m, 2H), 7.24-7.15 (m, 4H); ${ }^{13} \mathrm{C}$ NMR (100 MHz, $\left.\mathrm{CDCl}_{3}\right): \delta$ 194.5, 147.1, 145.2, 145.1, 144.6, 143.7, 139.6, 137.8, 134.9, 132.8, 131.8, 131.1, 129.5, 129.1, 129.0, 128.7, 128.3, 127.9, 127.8, 127.7, 127.1, 126.2, 125.1, 124.7, 122.8, 122.6, 121.6, 74.3; HRMS (ES ${ }^{+}$TOF) calcd for $\mathrm{C}_{28} \mathrm{H}_{18} \mathrm{BrO}$ $\left([\mathrm{M}+\mathrm{H}]^{+}\right):$449.0536, found 449.0534 .

\section{0-(4-chlorophenyl)-2-methyl-4b-phenylindeno[2,1-a]inden-5(4bH)-one (2x)}

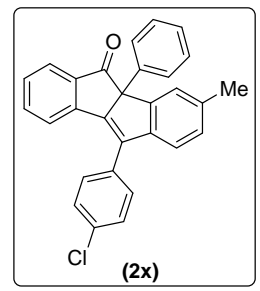

The reaction of 4-(4-chlorophenyl)-1,2-diphenyl-4-(p-tolyl)buta-2,3-dien-1-one $\mathbf{1 x}$ (84.2 mg, $0.2 \mathrm{mmol}, 1.0$ equiv), $\mathrm{FeCl}_{3}$ (65 mg, $0.4 \mathrm{mmol}, 2.0$ equiv), in $2 \mathrm{~mL}$ of DCM at $50{ }^{\circ} \mathrm{C}$ for $12 \mathrm{~h}$ afforded $2 \mathbf{x}$ (71.0 mg, 85\%) as a yellow solid; m.p. 181-183

${ }^{\circ} \mathrm{C}$ (Petroleum ether/EtOAc); $\mathrm{R} f=0.45$ (Petroleum ether/EtOAc $\left.=10 / 1\right) ;{ }^{1} \mathrm{H}$ NMR $(400 \mathrm{MHz}$, 
$\left.\mathrm{CDCl}_{3}\right): \delta 7.73(\mathrm{~d}, J=7.6 \mathrm{~Hz}, 1 \mathrm{H}), 7.65-7.61(\mathrm{~m}, 4 \mathrm{H}), 7.56(\mathrm{t}, J=8.8 \mathrm{~Hz}, 2 \mathrm{H}), 7.51(\mathrm{~d}, J=8.4 \mathrm{~Hz}$, 2H), 7.40 (t, $J=7.4 \mathrm{~Hz}, 1 \mathrm{H}), 7.24-7.16(\mathrm{~m}, 5 \mathrm{H}), 7.06$ (d, $J=7.6 \mathrm{~Hz}, 1 \mathrm{H}), 2.35$ (s, 3H); ${ }^{13} \mathrm{C}$ NMR $\left(100 \mathrm{MHz}, \mathrm{CDCl}_{3}\right): \delta 194.8,146.2,145.4,144.4,144.0,142.6,139.8,137.9,137.3,134.9,134.5$, 132.5, 130.8, 128.9, 128.8, 128.6, 128.1, 127.6, 126.2, 125.7, 125.1, 122.5, 121.3, 74.0, 21.6; HRMS $\left(\mathrm{ES}^{+}-\mathrm{TOF}\right)$ calcd for $\mathrm{C}_{29} \mathrm{H}_{20} \mathrm{ClO}\left([\mathrm{M}+\mathrm{H}]^{+}\right): 419.1197$, found 419.1194.

\section{0-(4-chlorophenyl)-2-methoxy-4b-phenylindeno[2,1-a]inden-5(4bH)-one (2y)}

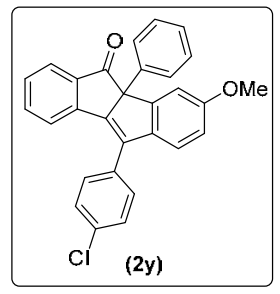

The reaction of 4-(4-chlorophenyl)-4-(4-methoxyphenyl)-1,2-diphenylbuta-2,3dien-1-one 1y (87.4 mg, $0.2 \mathrm{mmol}, 1.0$ equiv), $\mathrm{FeCl}_{3}$ (65 mg, $0.4 \mathrm{mmol}, 2.0$ equiv), in $2 \mathrm{~mL}$ of DCM at $50{ }^{\circ} \mathrm{C}$ for $12 \mathrm{~h}$ afforded $2 \mathbf{y}(55.1 \mathrm{mg}, 63 \%)$ as a yellow solid; m.p. $169-172{ }^{\circ} \mathrm{C}$ (Petroleum ether/EtOAc); $\mathrm{R} f=0.45$ (Petroleum ether/EtOAc $\left.=5 / 1\right) ;{ }^{1} \mathrm{H}$ NMR $(400$ $\left.\mathrm{MHz}, \mathrm{CDCl}_{3}\right): \delta 7.74(\mathrm{~d}, J=7.6 \mathrm{~Hz}, 1 \mathrm{H}), 7.64-7.62(\mathrm{~m}, 4 \mathrm{H}), 7.56-7.51(\mathrm{~m}, 3 \mathrm{H}), 7.42-7.38(\mathrm{~m}, 1 \mathrm{H})$, $7.32(\mathrm{~d}, J=2.4 \mathrm{~Hz}, 1 \mathrm{H}), 7.25-7.16(\mathrm{~m}, 5 \mathrm{H}), 6.81-6.78$ (m, 1H), 3.80 (s, 3H); ${ }^{13} \mathrm{C}$ NMR (100 MHz, $\left.\mathrm{CDCl}_{3}\right): \delta 194.8,159.6,147.1,145.2,144.22,144.17,139.8,138.0,137.9,134.9,134.5,132.5,130.8$, 129.0, 128.8, 127.9, 127.7, 126.1, 125.1, 122.3, 122.2, 113.3, 111.3, 73.8, 55.6; HRMS (ES ${ }^{+}$TOF) calcd for $\mathrm{C}_{29} \mathrm{H}_{20} \mathrm{ClO}_{2}\left([\mathrm{M}+\mathrm{H}]^{+}\right)$: 435.1146, found 435.1146 .

\section{3-(1-(4-isobutylphenyl)vinyl)-2-methyl-1H-inden-1-one (2z)}

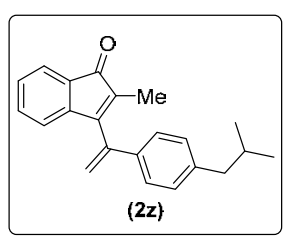

The reaction of 4-(4-isobutylphenyl)-2-methyl-1-phenylpenta-2,3-dien-1-one $\mathbf{1 z}$ (60.9 mg, $0.2 \mathrm{mmol}, 1.0$ equiv), $\mathrm{FeCl}_{3}$ (65 mg, $0.4 \mathrm{mmol}, 2.0$ equiv), in $2 \mathrm{~mL}$ of $\mathrm{DCM}$ at $50{ }^{\circ} \mathrm{C}$ for $12 \mathrm{~h}$ afforded $\mathbf{2 z}(43.0 \mathrm{mg}, 70 \%)$ as an orange liquid; $\mathrm{R} f=0.45$ $($ Petroleum ether/EtOAc $=10 / 1) ;{ }^{1} \mathrm{H}$ NMR $\left(400 \mathrm{MHz}, \mathrm{CDCl}_{3}\right): \delta 7.43(\mathrm{~d}, J=6.8 \mathrm{~Hz}, 1 \mathrm{H}), 7.32(\mathrm{~d}, J$ $=8.0 \mathrm{~Hz}, 2 \mathrm{H}), 7.18-7.10(\mathrm{~m}, 4 \mathrm{H}), 6.65(\mathrm{~d}, J=6.8 \mathrm{~Hz}, 1 \mathrm{H}), 5.91(\mathrm{~s}, 1 \mathrm{H}), 5.41(\mathrm{~s}, 1 \mathrm{H}), 2.47$ (d, $J=7.2$ $\mathrm{Hz}, 2 \mathrm{H}), 1.88-1.85(\mathrm{~m}, 1 \mathrm{H}), 1.79(\mathrm{~s}, 3 \mathrm{H}), 0.90$ (d, $J=6.8 \mathrm{~Hz}, 6 \mathrm{H}) ;{ }^{13} \mathrm{C}$ NMR $\left(100 \mathrm{MHz}, \mathrm{CDCl}_{3}\right): \delta$ 
198.3, 155.4, 146.1, 142.2, 141.2, 135.3, 133.3, 132.6, 130.8, 129.4, 127.8, 126.4, 122.2, 120.9, 116.3, 45.1, 30.1, 22.3, 8.7; HRMS (ES $\left.{ }^{+}-\mathrm{TOF}\right)$ calcd for $\mathrm{C}_{22} \mathrm{H}_{23} \mathrm{O}\left([\mathrm{M}+\mathrm{H}]^{+}\right)$: 303.1743, found 303.1749.

\section{Access to unsymmetrical dibenzo[a,e]pentalenes 4 from 2}

\section{(a) General procedure:}

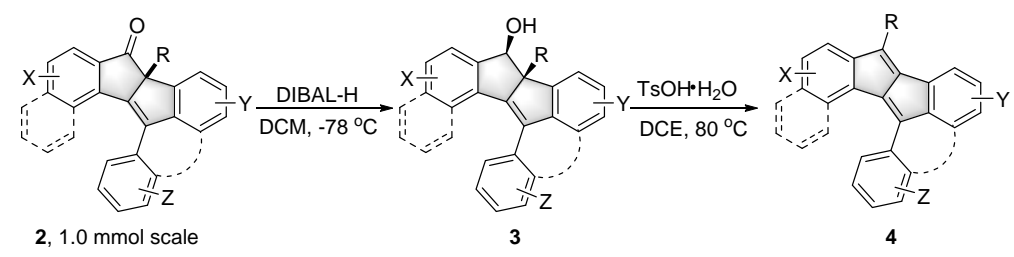

Step one: To a solution of indanone-fused polycyclic compounds 2 (1.0 mmol, 1.0 equiv) in $5 \mathrm{~mL}$ of dry DCM at $-78{ }^{\circ} \mathrm{C}$ under $\mathrm{N}_{2}$ was added dropwise DIBAL-H (1.5 M solution in toluene, $1.05 \mathrm{mmol}$, 1.05 equiv). After the solution was stirred at $-78{ }^{\circ} \mathrm{C}$ for $1.0 \mathrm{~h}$, the reaction was quenched by adding $10 \mathrm{~mL}$ of cold water and kept stirring for another $0.5 \mathrm{~h}$. Then the mixture was allowed to warm up to room temperature and extracted with DCM $(3 \times 20 \mathrm{~mL})$. The combined organic layers were dried over $\mathrm{Na}_{2} \mathrm{SO}_{4}$ and concentrated in vacuo. The residue was purified by flash silica gel chromatography to afford reduction products 3 with high regioselectivity.

Step two: The solution of 3 (0.3 mmol, 1.0 equiv) in $3 \mathrm{~mL}$ of DCE was added $\mathrm{TsOH}^{\circ} \mathrm{H}_{2} \mathrm{O}(0.6 \mathrm{mmol}$, 2.0 equiv). This mixture was allowed to stir at $80{ }^{\circ} \mathrm{C}$ for $12 \mathrm{~h}$. After completion, the reaction was quenched by adding $10 \mathrm{~mL}$ of $\mathrm{H}_{2} \mathrm{O}$ and extracted with DCM $(3 \times 10 \mathrm{~mL})$. The combined organic phase was washed with $\mathrm{H}_{2} \mathrm{O}(3 \times 10 \mathrm{~mL})$, dried over anhydrous $\mathrm{Na}_{2} \mathrm{SO}_{4}$, concentrated in vacuo and purified by flash silica gel chromatography to afford dibenzo[a,e]pentalenes 4 .

(b) Experiment data for 3 and 4:

cis-4b,10-diphenyl-4b,5-dihydroindeno[2,1-a]inden-5-ol (3a) 


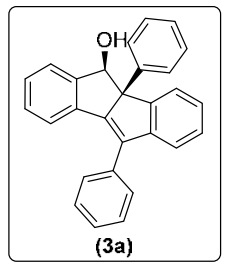

The solution of 4b,10-diphenylindeno[2,1-a]inden-5(4bH)-one 2a (370.4 mg, 1.0 mmol) and DIBAL-H (1.5 M solution in toluene, $0.7 \mathrm{~mL}, 1.05 \mathrm{mmol})$ in $5 \mathrm{~mL}$ of dry DCM afforded 3a (357.0 mg, 96\%) as a white solid; m.p. 97-98 ${ }^{\circ} \mathrm{C}$ (Petroleum ether/EtOAc); R $f=0.25$ (Petroleum ether/EtOAc = 10/1); ${ }^{1} \mathrm{H}$ NMR (400 MHz, CDCl3): $\delta 7.90$ (d, $J$ $=6.8 \mathrm{~Hz}, 1 \mathrm{H}), 7.65(\mathrm{~d}, J=8.0 \mathrm{~Hz}, 2 \mathrm{H}), 7.61-7.57(\mathrm{~m}, 3 \mathrm{H}), 7.52(\mathrm{t}, J=7.6 \mathrm{~Hz}, 2 \mathrm{H}), 7.43(\mathrm{t}, J=7.4$ Hz, 1H), 7.35-7.33 (m, 2H), 7.29-7.25 (m, 2H), 7.22-7.15 (m, 5H), $5.11(\mathrm{~d}, J=12.0 \mathrm{~Hz}, 1 \mathrm{H})$, 2.11-2.07 (m, 1H); ${ }^{13} \mathrm{C}$ NMR (100 MHz, CDCl3): $\delta$ 152.9, 150.7, 148.8, 146.7, 138.8, 136.1, 135.7, 134.3, 129.4, 129.2, 128.42, 128.35, 128.07, 128.05, 127.4, 127.2, 125.5, 124.5, 124.2, 121.7, 121.4, 77.3, 74.6; HRMS (ES ${ }^{+}$-TOF) calcd for $\mathrm{C}_{28} \mathrm{H}_{21} \mathrm{O}\left([\mathrm{M}+\mathrm{H}]^{+}\right)$: 373.1587, found 373.1596.

\section{5,10-diphenylindeno[2,1-a]indene (4a)}

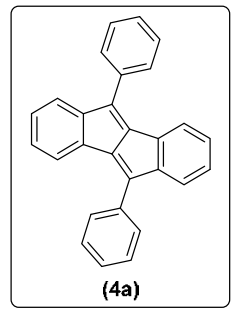

The solution of cis-4b,10-diphenyl-4b,5-dihydroindeno[2,1-a]inden-5-ol 3a (111.8 mg, $0.3 \mathrm{mmol}, 1.0$ equiv) and $\mathrm{TsOH}^{\cdot} \mathrm{H}_{2} \mathrm{O}$ (114 mg, $0.6 \mathrm{mmol}, 2.0$ equiv) in $3 \mathrm{~mL}$ of DCE afforded 4a (86.4 mg, 78\%) as a red solid; m.p. 237-238 ${ }^{\circ} \mathrm{C}$ (Petroleum ether/EtOAc); $\mathrm{R} f=0.35$ (Petroleum ether/EtOAc $=20 / 1) ;{ }^{1} \mathrm{H}$ NMR $\left(400 \mathrm{MHz}, \mathrm{CDCl}_{3}\right): \delta$ 7.68-7.66 (m, 2H), 7.52 (t, $J=7.4 \mathrm{~Hz}, 2 \mathrm{H}), 7.46-7.42(\mathrm{~m}, 1 \mathrm{H}), 7.21$ (d, $J=7.2 \mathrm{~Hz}, 1 \mathrm{H}), 7.02$ (d, $J=7.2 \mathrm{~Hz}$, 1H), 6.92-6.88 (m, 1H), 6.86-6.82 (m, 1H); $\left.{ }^{13} \mathrm{C} \mathrm{NMR} \mathrm{(100} \mathrm{MHz,} \mathrm{CDCl}_{3}\right): \delta 149.7,143.2,140.7$, 135.2, 133.9, 128.8, 128.6, 128.5, 127.8, 127.4, 122.5, 121.9; HRMS (ES ${ }^{+}$TOF) calcd for $\mathrm{C}_{28} \mathrm{H}_{19}$ $\left([\mathrm{M}+\mathrm{H}]^{+}\right): 355.1481$, found 355.1483. (Spectroscopic data match that previously reported ${ }^{3}$ )

\section{cis-8-chloro-4b,10-diphenyl-4b,5-dihydroindeno[2,1-a]inden-5-ol (3b)}

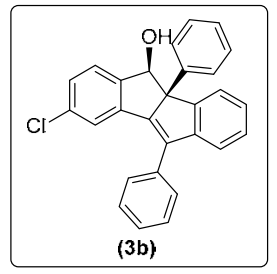

The solution of 8-chloro-4b,10-diphenylindeno[2,1-a]inden-5(4bH)-one 2e (409.8 mg, $1.0 \mathrm{mmol})$ and DIBAL-H (1.5 M solution in toluene, $0.7 \mathrm{~mL}, 1.05 \mathrm{mmol})$ in 5 mL of dry DCM afforded 3b (342.0 mg, 85\%) as a white solid; m.p. 176-177 ${ }^{\circ} \mathrm{C}$ 
(Petroleum ether/EtOAc); R $f=0.20($ Petroleum ether/EtOAc $=5 / 1) ;{ }^{1} \mathrm{H}$ NMR $\left(400 \mathrm{MHz}, \mathrm{CDCl}_{3}\right): \delta$ 7.85-7.83 (m, 1H), 7.65-7.63 (m, 2H), 7.57-7.50 (m, 5H) 7.46-7.42 (m, 1H), 7.36-7.35 (m, 2H), 7.31-7.26 (m, 2H), 7.24-7.18 (m, 4H), 5.12 (d, $J=11.2 \mathrm{~Hz}, 1 \mathrm{H}), 2.06-2.03(\mathrm{~m}, 1 \mathrm{H}) ;{ }^{13} \mathrm{C}$ NMR $(100$ $\left.\mathrm{MHz}, \mathrm{CDCl}_{3}\right): \delta 152.6,150.3,148.4,146.7,137.5,136.4,135.4,134.1,133.3,130.4,129.3,128.52$, 128.48, 128.4, 128.2, 127.6, 125.7, 124.4, 124.1, 121.9, 121.6, 77.2, 74.2; HRMS (ES ${ }^{+}$-TOF) calcd for $\mathrm{C}_{28} \mathrm{H}_{20} \mathrm{ClO}\left([\mathrm{M}+\mathrm{H}]^{+}\right)$: 407.1197, found 407.1184.

\section{3-chloro-5,10-diphenylindeno[2,1-a]indene (4b)}

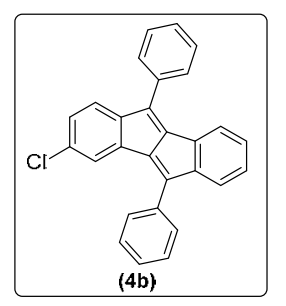

The solution of cis-8-chloro-4b,10-diphenyl-4b,5-dihydroindeno[2,1-a]inden-5-ol 3b (123.3 mg, $0.3 \mathrm{mmol}, 1.0$ equiv) and TsOH$\cdot \mathrm{H}_{2} \mathrm{O}$ (114 mg, $0.6 \mathrm{mmol}, 2.0$ equiv) in $3 \mathrm{~mL}$ of DCE afforded $\mathbf{4 b}$ (106.7 $\mathrm{mg}, 85 \%)$ a red solid; m.p. $219-222{ }^{\circ} \mathrm{C}$ (Petroleum ether/EtOAc); R $f=0.30$ (Petroleum ether/EtOAc $=20 / 1) ;{ }^{1} \mathrm{H}$ NMR $\left(400 \mathrm{MHz}, \mathrm{CDCl}_{3}\right): \delta$ 7.64 (d, $J=7.2 \mathrm{~Hz}, 2 \mathrm{H}), 7.58$ (d, $J=8.4 \mathrm{~Hz}, 2 \mathrm{H}), 7.52-7.42$ (m, 5H), 7.19 (d, $J=6.8 \mathrm{~Hz}, 1 \mathrm{H}), 7.14$ (d, $J=8.8 \mathrm{~Hz}, 1 \mathrm{H}), 7.01$ (d, $J=7.2 \mathrm{~Hz}, 1 \mathrm{H}), 6.94$ (d, $J=7.2 \mathrm{~Hz}, 1 \mathrm{H}), 6.89$ (t, $J=7.2 \mathrm{~Hz}, 2 \mathrm{H}), 6.83$ (d, $J=7.0 \mathrm{~Hz}, 2 \mathrm{H}) ;{ }^{13} \mathrm{C} \mathrm{NMR}\left(100 \mathrm{MHz}, \mathrm{CDCl}_{3}\right): \delta 149.7,149.3,143.5,143.0,141.1,139.1,135.0$, 134.9, 134.5, 133.7, 132.4, 129.8, 129.0, 128.9, 128.7, 128.5, 128.0, 127.8, 127.6, 122.6, 122.1, 122.0, 121.8; HRMS (ES ${ }^{+}$TOF) calcd for $\mathrm{C}_{28} \mathrm{H}_{17} \mathrm{ClNa}\left([\mathrm{M}+\mathrm{Na}]^{+}\right)$: 411.0911, found 411.0898.

\section{cis-4b,10-diphenyl-8-(trifluoromethyl)-4b,5-dihydroindeno[2,1-a]inden-5-ol (3c)}

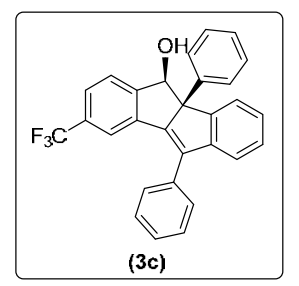

The solution of 4b,10-diphenyl-8-(trifluoromethyl)indeno[2,1-a]inden-5(4bH)-one 2h (442.5 mg, $1.0 \mathrm{mmol})$ and DIBAL-H (1.5 M solution in toluene, $0.7 \mathrm{~mL}, 1.05$ mmol) in $5 \mathrm{~mL}$ of dry DCM afforded 3c (404.0 mg, 90\%) as a white solid; m.p.

92-95 ${ }^{\circ} \mathrm{C}$ (Petroleum ether/EtOAc); Rf = 0.25 (Petroleum ether/EtOAc $\left.=5 / 1\right) ;{ }^{1} \mathrm{H}$ NMR $(400 \mathrm{MHz}$, $\left.\mathrm{CDCl}_{3}\right): \delta$ 7.91-7.89 (m, 1H), $7.79(\mathrm{~s}, 1 \mathrm{H}), 7.6-7.64(\mathrm{~m}, 2 \mathrm{H}), 7.58-7.53(\mathrm{~m}, 4 \mathrm{H})$, 7.48-7.44 (m, 3H), 
7.39-7.37 (m, 1H), 7.32-7.24 (m, 4H), 7.23-7.17 (m, 1H), 5.11 (d, $J=12.0 \mathrm{~Hz}, 1 \mathrm{H}), 2.22(\mathrm{~d}, J=12.0$ $\mathrm{Hz}, 1 \mathrm{H}) ;{ }^{13} \mathrm{C}$ NMR (100 MHz, $\left.\mathrm{CDCl}_{3}\right): \delta 156.3,148.7,146.3,138.2,138.1,136.2,133.6,130.6$ (q, $\left.{ }^{2} J_{\mathrm{C}-\mathrm{F}}=31.2 \mathrm{~Hz}\right), 129.2,129.0,128.63,128.58,127.7,127.6,126.1,124.9\left(\mathrm{q},{ }^{3} J_{\mathrm{C}-\mathrm{F}}=3.6 \mathrm{~Hz}\right), 124.6$, 124.5, $124.0\left(\mathrm{q},{ }^{1} J_{\mathrm{C}-\mathrm{F}}=270.8 \mathrm{~Hz}\right), 121.9,118.3$ (broad), 77.1, 74.8; HRMS (ES ${ }^{+}$TOF) calcd for $\mathrm{C}_{29} \mathrm{H}_{20} \mathrm{~F}_{3} \mathrm{O}\left([\mathrm{M}+\mathrm{H}]^{+}\right):$441.1461, found 441.1448.

\section{5,10-diphenyl-3-(trifluoromethyl)indeno[2,1-a]indene (4c)}

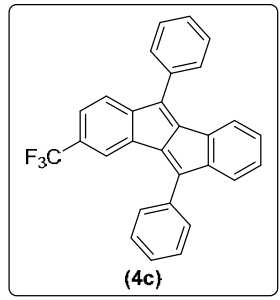

The solution of cis-4b,10-diphenyl-8-(trifluoromethyl)-4b,5-dihydroindeno[2,1-a] inden-5-ol 3c (133.4 mg, $0.3 \mathrm{mmol}, 1.0$ equiv) and $\mathrm{TsOH}^{\cdot} \mathrm{H}_{2} \mathrm{O}(114 \mathrm{mg}, 0.6 \mathrm{mmol}$, 2.0 equiv) in $3 \mathrm{~mL}$ of DCE afforded $4 \mathrm{c}(96.0 \mathrm{mg}, 72 \%)$ as a red solid; m.p. 207-210 ${ }^{\circ} \mathrm{C}$ (Petroleum ether/EtOAc); $\mathrm{R} f=0.30$ (Petroleum ether/EtOAc $\left.=20 / 1\right) ;{ }^{1} \mathrm{H}$ NMR $(400$ $\left.\mathrm{MHz}, \mathrm{CDCl}_{3}\right): \delta$ 7.67-7.64 (m, 4H), 7.57-7.47 (m, 6H), $7.38(\mathrm{~s}, 1 \mathrm{H}), 7.22$ (d, J = 7.2 Hz, 1H), 7.10 (d, $J=8.0 \mathrm{~Hz}, 1 \mathrm{H})$, 7.11-7.05 (m, 2H), 6.96-6.92 (m, 1H), 6.90-6.86 (m, 1H); ${ }^{13} \mathrm{C}$ NMR $(100 \mathrm{MHz}$, $\left.\mathrm{CDCl}_{3}\right): \delta 152.7,149.4,145.3,142.8,141.6,139.3,135.5,134.9,133.3\left(\mathrm{q},{ }^{3} J_{\mathrm{C}-\mathrm{F}}=2.4 \mathrm{~Hz}\right), 129.7$, 129.4, 129.1, 128.9, 128.8, 128.4, 128.0, 124.9 (q, $\left.{ }^{3} J_{\mathrm{C}-\mathrm{F}}=3.7 \mathrm{~Hz}\right), 124.3\left(\mathrm{q},{ }^{1} J_{\mathrm{C}-\mathrm{F}}=270.2 \mathrm{~Hz}\right), 123.1$, 121.9, 118.1 (broad); HRMS (ES ${ }^{+}$TOF) calcd for $\mathrm{C}_{29} \mathrm{H}_{17} \mathrm{~F}_{3} \mathrm{Na}\left([\mathrm{M}+\mathrm{Na}]^{+}\right)$: 445.1175, found 445.1186.

cis-7a,12-diphenyl-7,7a-dihydrobenzo[4,5]pentaleno[1,2-a]naphthalen-7-ol (3d)

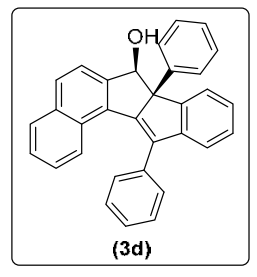
The solution of 7a,12-diphenylbenzo[4,5]pentaleno[1,2-a]naphthalen-7(7aH)-one $\mathbf{2 j}$ (420.5 mg, $1.0 \mathrm{mmol}$ ) and DIBAL-H (1.5 M solution in toluene, $0.7 \mathrm{~mL}, 1.05 \mathrm{mmol}$ ) in $5 \mathrm{~mL}$ of dry DCM afforded 3d (336.0 mg, 80\%) as a white solid; m.p. 207-208 ${ }^{\circ} \mathrm{C}$ (Petroleum ether/EtOAc); R $f=0.25$ (Petroleum ether/EtOAc $=5 / 1) ;{ }^{1} \mathrm{H}$ NMR $\left(400 \mathrm{MHz}, \mathrm{CDCl}_{3}\right): \delta$ 7.81-7.76 (m, 2H), 7.52 (d, $J=8.4 \mathrm{~Hz}, 1 \mathrm{H}), 7.38$ (d, $J=8.4 \mathrm{~Hz}, 1 \mathrm{H}), 7.30(\mathrm{t}, J=7.4 \mathrm{~Hz}, 1 \mathrm{H})$, 
7.23-7.12 (m, 6H), 7.05-6.96 (m, 4H), 6.93-6.90 (m, 2H), 6.86-6.73 (m, 2H), 5.68-5.64 (m, 1H), 4.48 (d, $J=6.4 \mathrm{~Hz}, 1 \mathrm{H}), 13.4$ (d, $J=11.6 \mathrm{~Hz}, 1 \mathrm{H}) ;{ }^{13} \mathrm{C}$ NMR $\left(100 \mathrm{MHz}, \mathrm{CDCl}_{3}\right): \delta 145.1,143.7,142.5$, 139.7, 138.9, 138.1, 137.8, 133.9, 130.3, 130.1, 129.5, 128.7, 128.2, 127.7, 127.3, 127.03, 126.99, 126.8, 126.5, 125.3, 125.1, 121.8, 76.5, 64.6; HRMS (ES ${ }^{+}$TOF) calcd for $\mathrm{C}_{32} \mathrm{H}_{23} \mathrm{O}\left([\mathrm{M}+\mathrm{H}]^{+}\right)$: 423.1743, found 423.1746 .

\section{7,12-diphenylbenzo[4,5]pentaleno[1,2-a]naphthalene (4d)}

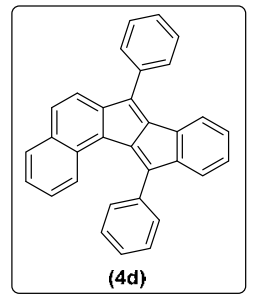

The solution of cis-7a,12-diphenyl-7,7a-dihydrobenzo[4,5]pentaleno[1,2-a] naphthalen-7-ol 3d (126.8 mg, $0.3 \mathrm{mmol}, 1.0$ equiv) and $\mathrm{BF}_{3} \mathrm{Et}_{2} \mathrm{O}(0.6 \mathrm{mmol}, 2.0$ equiv) in $3 \mathrm{~mL}$ of DCE afforded $\mathbf{4 d}$ (103.9 mg, 82\%) as a red solid; m.p. 218-220 ${ }^{\circ} \mathrm{C}$

(Petroleum ether/EtOAc); R $f=0.30$ (Petroleum ether/EtOAc $=20 / 1) ;{ }^{1} \mathrm{H}$ NMR $\left(400 \mathrm{MHz}, \mathrm{CDCl}_{3}\right): \delta$ 7.72-7.62 (m, 5H), 7.45-7.31 (m, 8H), 7.25-7.17 (m, 4H), 7.07 (t, $J=7.4$ Hz, 1H), 6.75 (t, $J=7.6$ Hz, 1H), 6.50 (s, $1 \mathrm{H}) ;{ }^{13} \mathrm{C}$ NMR (100 MHz, $\left.\mathrm{CDCl}_{3}\right): \delta 149.6,143.5,142.6,142.4,141.6,136.0,133.2$, 132.7, 132.6, 131.8, 131.4, 129.3, 129.2, 128.9, 128.8, 128.7, 128.6, 128.5, 128.4, 128.3, 128.12, 128.06, 127.7, 127.5, 126.2, 124.9, 123.5, 119.5; HRMS $\left(\mathrm{ES}^{+}-\mathrm{TOF}\right)$ calcd for $\mathrm{C}_{32} \mathrm{H}_{21}\left([\mathrm{M}+\mathrm{H}]^{+}\right)$: 405.1638, found 405.1643.

\section{cis-4b-(4-bromophenyl)-8-methyl-10-phenyl-4b,5-dihydroindeno[2,1-a]inden-5-ol (3e)}

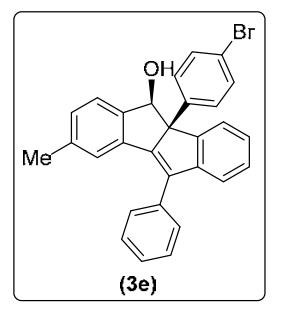

The solution of 4b-(4-bromophenyl)-8-methyl-10-phenylindeno[2,1-a]inden5(4bH)-one 2p (444.5 mg, $1.0 \mathrm{mmol})$ and DIBAL-H (1.5 M solution in toluene, $0.7 \mathrm{~mL}, 1.05 \mathrm{mmol})$ in $5 \mathrm{~mL}$ of dry DCM afforded $3 \mathbf{e}(414.0 \mathrm{mg}, 93 \%)$ as a white solid; m.p. $168-170{ }^{\circ} \mathrm{C}$ (Petroleum ether/EtOAc); $\mathrm{R} f=0.25$ (Petroleum ether/EtOAc $=20 / 1$ ); ${ }^{1} \mathrm{H}$ NMR (400 MHz, $\left.\mathrm{CDCl}_{3}\right): \delta$ 7.83-7.81 (m, 1H), $7.64(\mathrm{~d}, J=7.2 \mathrm{~Hz}, 2 \mathrm{H}), 7.53(\mathrm{t}, J=7.6 \mathrm{~Hz}, 2 \mathrm{H})$, 7.47-7.42 (m, 3H), 7.36-7.32 (m, 4H),7.29-7.26 (m, 2H), 7.24-7.22 (m, 1H), 7.03 (d, $J=7.6 \mathrm{~Hz}, 1 \mathrm{H})$, 
$5.08(\mathrm{~d}, J=11.6 \mathrm{~Hz}, 1 \mathrm{H}), 2.30(\mathrm{~s}, 3 \mathrm{H}), 2.01-1.97(\mathrm{~m}, 1 \mathrm{H}) ;{ }^{13} \mathrm{C} \mathrm{NMR}\left(100 \mathrm{MHz}, \mathrm{CDCl}_{3}\right): \delta 150.3$, 149.9, 148.3, 146.7, 138.4, 138.2, 136.0, 135.5, 134.2, 131.3, 130.8, 129.4, 129.1, 128.5, 128.2, 127.6, 125.6, 124.3, 123.8, 122.5, 121.5, 121.4, 74.4, 21.6; HRMS (ES ${ }^{+}$-TOF) calcd for $\mathrm{C}_{29} \mathrm{H}_{21} \mathrm{BrNaO}\left([\mathrm{M}+\mathrm{Na}]^{+}\right)$: 487.0668, found 487.0666.

\section{0-(4-bromophenyl)-3-methyl-5-phenylindeno[2,1-a]indene (4e)}

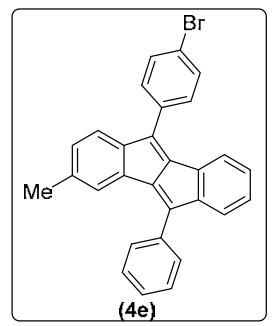

The solution of cis-4b-(4-bromophenyl)-8-methyl-10-phenyl-4b,5-dihydroindeno [2,1-a]inden-5-ol 3e (133.8 mg, $0.3 \mathrm{mmol}, 1.0$ equiv) and $\mathrm{TsOH} \cdot \mathrm{H}_{2} \mathrm{O}(114 \mathrm{mg}, 0.6$ mmol, 2.0 equiv) in $3 \mathrm{~mL}$ of DCE afforded $4 \mathbf{e}(84.8 \mathrm{mg}, 63 \%)$ as a red solid; m.p.

197-199 ${ }^{\circ} \mathrm{C}$ (Petroleum ether/EtOAc); $\mathrm{R} f=0.30$ (Petroleum ether/EtOAc $\left.=20 / 1\right) ;{ }^{1} \mathrm{H}$ NMR $(400$ $\left.\mathrm{MHz}, \mathrm{CDCl}_{3}\right): \delta$ 7.66-7.63 (m, 4H), 7.54-7.51 (m, 4H), 7.45 (t, $\left.J=7.2 \mathrm{~Hz}, 1 \mathrm{H}\right), 7.14$ (d, $J=6.8 \mathrm{~Hz}$, 1H), $7.01(\mathrm{~d}, J=6.8 \mathrm{~Hz}, 2 \mathrm{H}), 6.91-6.82(\mathrm{~m}, 3 \mathrm{H}), 6.70(\mathrm{~d}, J=7.6 \mathrm{~Hz}, 1 \mathrm{H}), 2.16(\mathrm{~s}, 3 \mathrm{H}) ;{ }^{13} \mathrm{C} \mathrm{NMR}$ (100 MHz, $\left.\mathrm{CDCl}_{3}\right): \delta 149.6,146.6,143.0,142.9,140.7,139.5,137.6,135.3,135.0,133.9,133.0$. 131.9, 130.1, 128.8, 128.7, 128.5, 128.2, 127.8, 127.4, 123.2, 122.7, 122.5, 122.0, 121.7, 21.4; HRMS (ES ${ }^{+}$-TOF) calcd for $\mathrm{C}_{29} \mathrm{H}_{20} \mathrm{Br}\left([\mathrm{M}+\mathrm{H}]^{+}\right)$: 447.0743, found 447.0753.

\section{cis-9b-phenyl-5,6,9b,10-tetrahydrodibenzo[cd,h]indeno[1,2-a]azulen-10-ol (3f)}

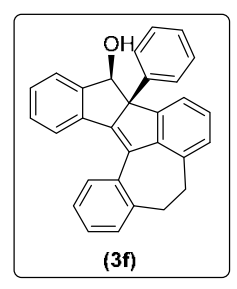

The solution of 9b-phenyl-6,9b-dihydrodibenzo[cd,h]indeno[1,2-a]azulen-10(5H)one $2 s$ (400.5 mg, $1.0 \mathrm{mmol})$ and DIBAL-H (1.5 M solution in toluene, $0.7 \mathrm{~mL}, 1.05$ mmol) in $5 \mathrm{~mL}$ of dry DCM afforded $3 \mathbf{f}(352.0 \mathrm{mg}$, 88\%) as a white solid; m.p.

153-156 ${ }^{\circ} \mathrm{C}$ (Petroleum ether/EtOAc); R $f=0.25$ (Petroleum ether/EtOAc $\left.=5 / 1\right) ;{ }^{1} \mathrm{H}$ NMR $(400 \mathrm{MHz}$, $\left.\mathrm{CDCl}_{3}\right): \delta$ 8.04-7.66 (m, 3H), 7.61-7.46 (m, 2H), 7.36 (d, $\left.J=7.6 \mathrm{~Hz}, 1 \mathrm{H}\right)$, 7.28-7.07 (m, 9H), 7.02 (d, $J=7.6 \mathrm{~Hz}, 1 \mathrm{H}), 5.12(\mathrm{~s}, 1 \mathrm{H}), 3.30-2.83(\mathrm{~m}, 4 \mathrm{H}), 2.30-2.03(\mathrm{~m}, 1 \mathrm{H}) ;{ }^{13} \mathrm{C} \mathrm{NMR}\left(100 \mathrm{MHz}, \mathrm{CDCl}_{3}\right): \delta$ 153.6 (broad), 149.0 (broad), 143.5, 142.5, 139.3, 138.0, 136.1, 133.8, 129.3, 129.1, 128.6, 128.5, 
128.3, 128.2, 127.1, 125.6, 124.9, 124.1, 121.9 (broad), 77.3, 35.6 (broad), 34.1; HRMS (ES ${ }^{+}$-TOF) calcd for $\mathrm{C}_{30} \mathrm{H}_{23} \mathrm{O}\left([\mathrm{M}+\mathrm{H}]^{+}\right)$: 399.1743, found 399.1740.

\section{0-phenyl-5,6-dihydrodibenzo[cd,h]indeno[1,2-a]azulene (4f)}

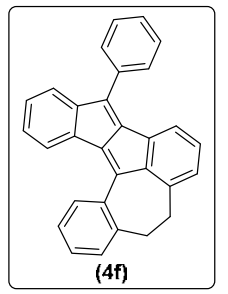

The solution of cis-9b-phenyl-5,6,9b,10-tetrahydrodibenzo[cd,h]indeno[1,2-a]azulen10-ol 3f (119.6 mg, 0.3 mmol, 1.0 equiv) and TsOH$\cdot \mathrm{H}_{2} \mathrm{O}$ (114 mg, $0.6 \mathrm{mmol}, 2.0$ equiv) in $3 \mathrm{~mL}$ of DCE afforded $\mathbf{4 f}$ (108.0 $\mathrm{mg}, 91 \%)$ as a red solid; m.p. $185-186{ }^{\circ} \mathrm{C}$ (Petroleum ether/EtOAc); R $f=0.30$ (Petroleum ether/EtOAc $=20 / 1) ;{ }^{1} \mathrm{H}$ NMR $\left(400 \mathrm{MHz}, \mathrm{CDCl}_{3}\right): \delta$ 7.98-7.92 (m, 1H), 7.66-7.63 (m, 2H), 7.52-7.46 (m, 3H), 7.42 (t, J = 7.2 Hz, 1H), 7.32-7.28 (m, 2H), 7.23-7.18 (m, 1H), 7.06-6.99 (m, 2H), 6.92-6.85 (m, 2H), 6.74-6.63 (m, 2H), 3.40-3.92 (m, 2H), 2.90-2.83 (m, 2H); ${ }^{13} \mathrm{C}$ NMR (100 MHz, $\left.\mathrm{CDCl}_{3}\right): \delta 149.9,146.5,143.4,143.2,142.5,142.2,139.8$, 139.1, 135.5, 135.3, 133.9, 133.7, 131.2, 129.6, 129.5, 129.4, 128.6, 127.6, 127.4, 127.0, 126.0, 122.3, 119.5, 35.6, 33.4; HRMS (ES ${ }^{+}$-TOF) calcd for $\mathrm{C}_{30} \mathrm{H}_{21}\left([\mathrm{M}+\mathrm{H}]^{+}\right)$: 381.1638, found 381.1627. cis-8-bromo-3-methyl-4b-phenyl-10-(p-tolyl)-4b,5-dihydroindeno[2,1-a]inden-5-ol (3g)

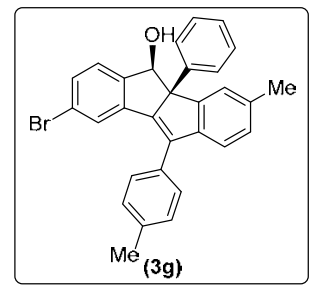

The solution of 8-bromo-3-methyl-4b-phenyl-10-(p-tolyl)indeno[2,1-a]inden5(4bH)-one 2t (476.5 mg, $1.0 \mathrm{mmol})$ and DIBAL-H (1.5 M solution in toluene, $0.7 \mathrm{~mL}, 1.05 \mathrm{mmol})$ in $5 \mathrm{~mL}$ of dry DCM afforded $3 \mathrm{~g}(444.0 \mathrm{mg}, 93 \%)$ as a white solid; m.p. 226-228 ${ }^{\circ} \mathrm{C}$ (Petroleum ether/EtOAc); Rf = 0.25 (Petroleum ether/EtOAc $\left.=5 / 1\right) ;{ }^{1} \mathrm{H}$ NMR (400 MHz, $\left.\mathrm{CDCl}_{3}\right): \delta$ 7.70-7.68 (m, 2H), 7.57 (d, $\left.J=7.2 \mathrm{~Hz}, 2 \mathrm{H}\right), 7.51(\mathrm{~d}, J=8.0 \mathrm{~Hz}, 2 \mathrm{H})$, 7.34 (d, $J=7.6 \mathrm{~Hz}, 2 \mathrm{H}), 7.29-7.24$ (m, 4H), 7.20-7.16 (m, 2H), 7.09 (d, $J=7.6 \mathrm{~Hz}, 1 \mathrm{H}), 5.01$ (d, $J=$ $8.0 \mathrm{~Hz}, 1 \mathrm{H}), 2.46$ (s, 3H), 2.43 (s, 3H), 2.06 (d, $J=12.0 \mathrm{~Hz}, 1 \mathrm{H}) ;{ }^{13} \mathrm{C}$ NMR $\left(100 \mathrm{MHz}, \mathrm{CDCl}_{3}\right): \delta$ 151.5, 148.9, 147.5, 143.9, 138.6, 138.3, 137.8, 137.7, 135.8, 130.9, 130.5, 129.3, 129.2, 129.0, 128.5, 128.2, 127.4, 126.4, 125.52, 125.49, 124.5, 122.3, 121.5, 74.3, 21.7, 21.4; HRMS (ES ${ }^{+}$-TOF) 
calcd for $\mathrm{C}_{30} \mathrm{H}_{24} \mathrm{BrO}\left([\mathrm{M}+\mathrm{H}]^{+}\right)$: 479.1005, found 479.0992 .

\section{8-bromo-2-methyl-5-phenyl-10-(p-tolyl)indeno[2,1-a]indene (4g)}

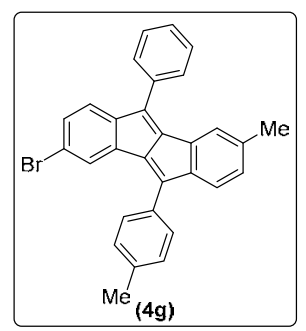

The solution of cis-8-bromo-3-methyl-4b-phenyl-10-(p-tolyl)-4b,5-dihydroindeno [2,1-a]inden-5-ol 3g (143.5 mg, $0.3 \mathrm{mmol}, 1.0$ equiv) and $\mathrm{TsOH} \cdot \mathrm{H}_{2} \mathrm{O}$ (114 mg, $0.6 \mathrm{mmol}, 2.0$ equiv) in $3 \mathrm{~mL}$ of DCE afforded $\mathbf{4 g}$ (107.6 $\mathrm{mg}, 75 \%)$ as a red solid; m.p. $227-229{ }^{\circ} \mathrm{C}($ Petroleum ether/EtOAc); $\mathrm{R} f=0.30$ (Petroleum ether/EtOAc $=$ 20/1); ${ }^{1} \mathrm{H}$ NMR (400 MHz, $\left.\mathrm{CDCl}_{3}\right): \delta 7.62$ (d, $\left.J=7.2 \mathrm{~Hz}, 2 \mathrm{H}\right), 7.54-7.50$ (m, 4H), 7.44 (t, $J=7.8 \mathrm{~Hz}$, 1H), 7.35-7.43 (m, 3H), 7.01-7.00 (m, 2H), 6.91 (d, $J=7.6 \mathrm{~Hz}, 1 \mathrm{H}), 6.85$ (d, $J=7.6 \mathrm{~Hz}, 1 \mathrm{H}), 6.71(\mathrm{~d}$, $J=7.6 \mathrm{~Hz}, 1 \mathrm{H}), 2.46$ (s, 3H), 2.16 (s, 3H); ${ }^{13} \mathrm{C}$ NMR (100 MHz, $\left.\mathrm{CDCl}_{3}\right): \delta$ 148.3, 146.7, 143.3, 142.8, 140.7, 139.3, 139.2, 137.9, 137.2, 135.5, 133.7, 130.6, 129.8, 129.5, 128.8, 128.7, 128.4, 128.31, 128.27, 124.6, 123.20, 123.16, 122.7, 121.4, 21.5, 21.4; HRMS (ES ${ }^{+}$-TOF) calcd for $\mathrm{C}_{30} \mathrm{H}_{22} \mathrm{Br}\left([\mathrm{M}+\mathrm{H}]^{+}\right):$461.0899, found 461.0885 .

\section{cis-10-(4-chlorophenyl)-2-methoxy-4b-phenyl-4b,5-dihydroindeno[2,1-a]inden-5-ol (3h)}

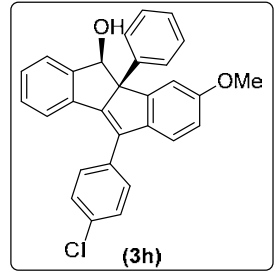

The solution of 10-(4-chlorophenyl)-2-methoxy-4b-phenylindeno[2,1-a]inden5(4bH)-one 2x (434.9 mg, $1.0 \mathrm{mmol})$ and DIBAL-H (1.5 M solution in toluene, $0.7 \mathrm{~mL}, 1.05 \mathrm{mmol})$ in $5 \mathrm{~mL}$ of dry DCM afforded $3 \mathbf{h}$ (365.3 $\mathrm{mg}, 84 \%)$ as a white solid; m.p. 107-109 ${ }^{\circ} \mathrm{C}$ (Petroleum ether/EtOAc); $\mathrm{R} f=0.25$ (Petroleum ether/EtOAc $\left.=5 / 1\right) ;{ }^{1} \mathrm{H}$ NMR (400 MHz, $\left.\mathrm{CDCl}_{3}\right): \delta$ 7.58-7.54 (m, 4H), 7.51-7.47 (m, 4H), 7.36-7.34 (m, 1H), 7.24-7.16 (m, 6H), $6.81\left(\mathrm{dd}, J_{1}=8.4 \mathrm{~Hz}, J_{2}=2.4 \mathrm{~Hz}, 1 \mathrm{H}\right), 5.12(\mathrm{~d}, J=7.6 \mathrm{~Hz}, 1 \mathrm{H}), 3.87$ (s, 3H), 2.07 (d, $J=12.0 \mathrm{~Hz}$, 1H); ${ }^{13} \mathrm{C}$ NMR (100 MHz, $\left.\mathrm{CDCl}_{3}\right): \delta 158.5,152.5,150.5,149.4,139.4,138.7,135.6,134.7,133.8$, 133.0, 130.6, 129.0, 128.7, 128.4, 127.9, 127.3, 124.2, 121.6, 121.3, 112.2, 112.1, 74.4, 55.7; HRMS (ES ${ }^{+}$-TOF) calcd for $\mathrm{C}_{29} \mathrm{H}_{22} \mathrm{ClO}_{2}\left([\mathrm{M}+\mathrm{H}]^{+}\right)$: 437.1303, found 437.1311. 


\section{0-(4-chlorophenyl)-2-methoxy-5-phenylindeno[2,1-a]indene (4h)}

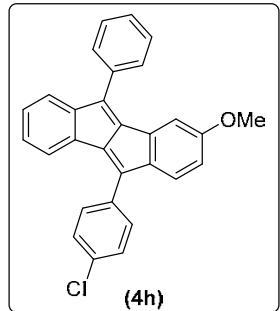

The solution of cis-10-(4-chlorophenyl)-2-methoxy-4b-phenyl-4b,5dihydroindeno[2,1-a]inden-5-ol $3 \mathbf{h}$ (131.1 $\mathrm{mg}, \quad 0.3 \mathrm{mmol}, 1.0$ equiv) and TsOH$\cdot \mathrm{H}_{2} \mathrm{O}$ (114 mg, $0.6 \mathrm{mmol}, 2.0$ equiv) in $3 \mathrm{~mL}$ of DCE afforded $4 \mathbf{h}$ (78.7 mg,

$60 \%$ ) as red solid; m.p. 204-206 ${ }^{\circ} \mathrm{C}$ (Petroleum ether/EtOAc); $\mathrm{R} f=0.30$ (Petroleum ether/EtOAc $=$ 10/1); ${ }^{1} \mathrm{H}$ NMR (400 MHz, $\left.\mathrm{CDCl}_{3}\right): \delta$ 7.66-7.59 (m, 4H), 7.53-7.43(m, 5H), 7.15 (d, $\left.J=7.2 \mathrm{~Hz}, 1 \mathrm{H}\right)$, 7.03 (d, $J=7.6 \mathrm{~Hz}, 1 \mathrm{H}), 6.92-6.84(\mathrm{~m}, 4 \mathrm{H}), 6.39-6.36$ (m, 1H), 3.71 (s, 3H); ${ }^{13} \mathrm{C}$ NMR $(100 \mathrm{MHz}$, $\left.\mathrm{CDCl}_{3}\right): \delta 160.2,149.2,142.4,142.2,142.1,140.9,139.8,137.0,135.0,134.5,133.8,132.5,129.8$, 129.0, 128.8, 128.7, 128.5, 128.4, 127.5, 122.7, 122.6, 121.4, 110.6, 110.4, 55.4; HRMS (ES ${ }^{+}$-TOF) calcd for $\mathrm{C}_{29} \mathrm{H}_{20} \mathrm{ClO}\left([\mathrm{M}+\mathrm{H}]^{+}\right)$: 419.1197, found 419.1183.

\section{Diverse transformations of 2 for the synthesis of 3D-polycyclic compounds 5-10}

\section{(a) General procedure for the synthesis of compounds 5:}
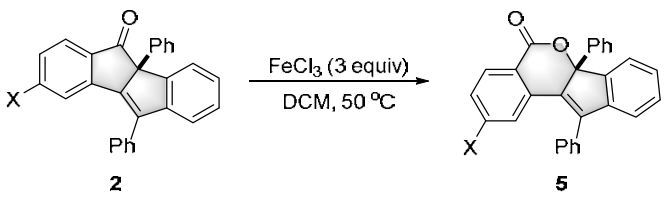

The solution of indanone-fused polycyclic compounds $2(0.2 \mathrm{mmol}, 1.0$ equiv) in $2 \mathrm{~mL}$ of DCM was added $\mathrm{FeCl}_{3}$ (97 mg, $0.6 \mathrm{mmol}$, 3.0 equiv). Then the vessel was sealed and submerged in an oil bath preheated to $50{ }^{\circ} \mathrm{C}$ for $2.5 \mathrm{~h}$. After completion, the mixture was quenched by adding $10 \mathrm{~mL}$ of $\mathrm{H}_{2} \mathrm{O}$ and extracted with DCM $(3 \times 10 \mathrm{~mL})$. The combined organic phase was washed with $\mathrm{H}_{2} \mathrm{O}(3 \times 10 \mathrm{~mL})$, dried over anhydrous $\mathrm{Na}_{2} \mathrm{SO}_{4}$, concentrated in vacuo and purified by flash silica gel chromatography to afford polycyclic compounds 5 .

Controlled experiments were carried out: (1) 2a could be transformed to lactam 5a in 93\% yield 
under $\mathrm{N}_{2}$ protection condition (without $\mathrm{O}_{2}$ ). (2) Most of starting material 2a was recovered when the reaction was conducted in dry DCM, $4 \AA \mathrm{MS}$ and $\mathrm{O}_{2}$ balloon condition. These results indicated that the $\mathrm{O}$ of 5 a might from $\mathrm{H}_{2} \mathrm{O}$ in solvent.
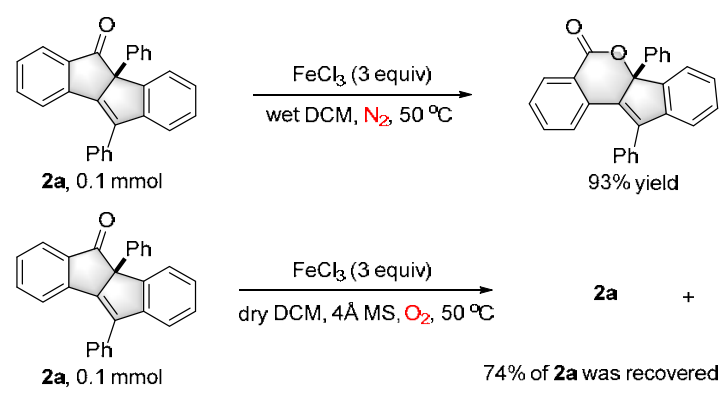

\section{(b) Experiment data for 5:}

\section{6a,11-diphenylindeno[1,2-c]isochromen-5(6aH)-one (5a)}

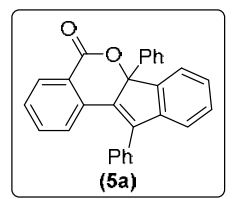

The solution of 4b,10-diphenylindeno[2,1-a]inden-5(4bH)-one 2a (74.0 mg, $0.2 \mathrm{mmol}$, 1.0 equiv) and $\mathrm{FeCl}_{3}\left(97 \mathrm{mg}, 0.6 \mathrm{mmol}, 3.0\right.$ equiv) in $2 \mathrm{~mL}$ of $\mathrm{DCM}$ at $50{ }^{\circ} \mathrm{C}$ for $2.5 \mathrm{~h}$ afford 5a (66.8 mg, 86\%) as a white solid; m.p. 223-226 ${ }^{\circ} \mathrm{C}$ (Petroleum ether/EtOAc); $\mathrm{R} f=0.25$ $($ Petroleum ether/EtOAc $=10 / 1) ;{ }^{1} \mathrm{H}$ NMR $\left(400 \mathrm{MHz}, \mathrm{CDCl}_{3}\right): \delta 8.04(\mathrm{~d}, J=7.6 \mathrm{~Hz}, 1 \mathrm{H}), 7.55-7.45$ (m, 7H), $7.41(\mathrm{~d}, J=6.8 \mathrm{~Hz}, 1 \mathrm{H}), 7.31-7.27(\mathrm{~m}, 3 \mathrm{H}), 7.23-7.17(\mathrm{~m}, 6 \mathrm{H}) ;{ }^{13} \mathrm{C} \mathrm{NMR}(100 \mathrm{MHz}$, $\left.\mathrm{CDCl}_{3}\right): \delta 166.2,146.5,143.4,142.6,139.8,135.7,133.6,133.3,132.8,131.1,129.3,129.1,128.9$, 128.7, 128.5, 128.0, 127.7, 125.5, 124.6, 124.1, 123.7, 121.8, 91.6; HRMS (ES ${ }^{+}$-TOF) calcd for $\mathrm{C}_{28} \mathrm{H}_{19} \mathrm{O}_{2}\left([\mathrm{M}+\mathrm{H}]^{+}\right): 387.1380$, found 387.1385.

\section{2-methoxy-6a,11-diphenylindeno[1,2-c]isochromen-5(6aH)-one (5b)}

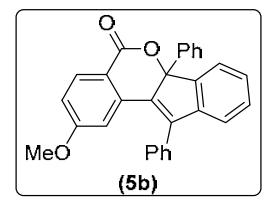

The solution of 8-methoxy-4b,10-diphenylindeno[2,1-a]inden-5(4bH)-one 2c (80.2 mg, $0.2 \mathrm{mmol}, 1.0$ equiv) and $\mathrm{FeCl}_{3}(97 \mathrm{mg}, 0.6 \mathrm{mmol}, 3.0$ equiv) in $2 \mathrm{~mL}$ of $\mathrm{DCM}$ at $50{ }^{\circ} \mathrm{C}$ for $2.5 \mathrm{~h}$ afford $5 \mathbf{b}(74.2 \mathrm{mg}, 89 \%)$ as a white solid; m.p. $169-171{ }^{\circ} \mathrm{C}$ (Petroleum ether/EtOAc); $\mathrm{R} f=0.4($ Petroleum ether/EtOAc $=5 / 1) ;{ }^{1} \mathrm{H}$ NMR $\left(400 \mathrm{MHz}, \mathrm{CDCl}_{3}\right): \delta 7.97(\mathrm{~d}, J=$ 
8.8 Hz, 1H), 7.55-7.47 (m, 7H), 7.40 (d, $J=6.8 \mathrm{~Hz}, 1 \mathrm{H}), 7.27-7.14$ (m, 6H), 6.76-6.73 (m, 1H), 6.61 (d, $J=2.4 \mathrm{~Hz}, 1 \mathrm{H}), 3.53$ (s, 3H); ${ }^{13} \mathrm{C}$ NMR (100 MHz, $\left.\mathrm{CDCl}_{3}\right): \delta$ 166.0, 163.4, 146.7, 143.2, 142.7, 139.9, 136.1, 135.1, 133.2, 132.8, 129.2, 129.0, 128.9, 128.8, 127.9, 127.7, 124.6, 124.0, 121.8, 116.3, 115.3, 109.4, 91.5, 55.1; HRMS (ES $\left.{ }^{+}-\mathrm{TOF}\right)$ calcd for $\mathrm{C}_{29} \mathrm{H}_{21} \mathrm{O}_{3}\left([\mathrm{M}+\mathrm{H}]^{+}\right)$: 417.1485, found 417.1494.

\section{6a,11-diphenyl-2-(trifluoromethyl)indeno[1,2-c]isochromen-5(6aH)-one (5c)}

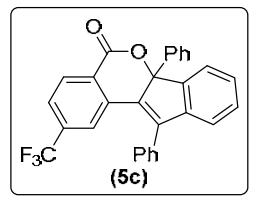

The solution of 4b,10-diphenyl-8-(trifluoromethyl)indeno[2,1-a]inden-5(4bH)-one 2h (87.6 mg, $0.2 \mathrm{mmol}, 1.0$ equiv) and $\mathrm{FeCl}_{3}$ (97 mg, $0.6 \mathrm{mmol}, 3.0$ equiv) in $2 \mathrm{~mL}$ of DCM at $50{ }^{\circ} \mathrm{C}$ for $2.5 \mathrm{~h}$ afford 5c (47.2 mg, 52\%) as a white solid; m.p. 209-211 ${ }^{\circ} \mathrm{C}$ (Petroleum ether/EtOAc); R $f=0.35$ (Petroleum ether/EtOAc = 10/1); ${ }^{1} \mathrm{H}$ NMR $\left(400 \mathrm{MHz}, \mathrm{CDCl}_{3}\right): \delta 8.16(\mathrm{~d}, J$ $=8.0 \mathrm{~Hz}, 1 \mathrm{H})$, 7.56-7.54 (m, 5H), 7.50-7.48 (m, 3H), 7.43-7.42 (m, 2H), 7.31-7.27 (m, 3H), 7.25$7.20(\mathrm{~m}, 3 \mathrm{H}) ;{ }^{13} \mathrm{C}$ NMR $\left(100 \mathrm{MHz}, \mathrm{CDCl}_{3}\right): \delta 165.0,146.5,144.6,142.9,139.2,135.0\left(\mathrm{q},{ }^{2} J_{\mathrm{C}-\mathrm{F}}=\right.$ 32.7 Hz), 134.3, 134.0, 131.9, 131.8, 129.6, 129.5, 129.3, 129.1, 128.5, 128.33, 128.30, 126.4, 124.9 $\left(\mathrm{q},{ }^{3} J_{\mathrm{C}-\mathrm{F}}=3.2 \mathrm{~Hz}\right), 123.0\left(\mathrm{q},{ }^{1} J_{\mathrm{C}-\mathrm{F}}=271.6 \mathrm{~Hz}\right), 122.5\left(\mathrm{q},{ }^{3} J_{\mathrm{C}-\mathrm{F}}=3.1 \mathrm{~Hz}\right), 122.3,91.8$; HRMS (ES ${ }^{+}$-TOF) calcd for $\mathrm{C}_{29} \mathrm{H}_{18} \mathrm{~F}_{3} \mathrm{O}_{2}\left([\mathrm{M}+\mathrm{H}]^{+}\right)$: 455.1253, found 455.1257.

\section{Synthesis of compound 6:}

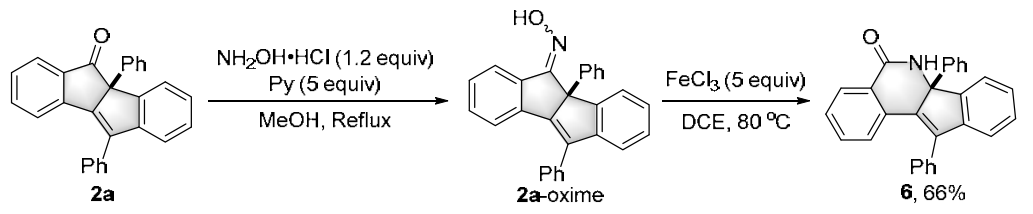

\section{6a,11-diphenyl-6,6a-dihydro-5H-indeno[1,2-c]isoquinolin-5-one (6)}

The solution of 4b,10-diphenylindeno[2,1-a]inden-5(4bH)-one 2a (74 mg, $0.2 \mathrm{mmol}, 1.0$ equiv) in 5 $\mathrm{mL}$ of $\mathrm{MeOH}$ was added pyridine (79 mg, $1.0 \mathrm{mmol}, 5.0$ equiv) and hydroxylamine hydrochloride (18 mg, $0.24 \mathrm{mmol}, 1.2$ equiv). Then the vessel was sealed and submerged in an oil bath preheated to 
$80{ }^{\circ} \mathrm{C}$ for $24 \mathrm{~h}$. After completion of the reaction, the solvent was removed under reduced pressure. 10 $\mathrm{mL}$ of $\mathrm{H}_{2} \mathrm{O}$ was added to the residual solid and extracted with DCM (3×10 mL). The combined organic layers were dried over $\mathrm{Na}_{2} \mathrm{SO}_{4}$ and concentrated in vacuo. The residue was purified with flash silica gel chromatography to afford 2a-oxime as a solid, which was used for the next step. To a solution of 2a-oxime in $16 \mathrm{~mL}$ of DCE were added $\mathrm{FeCl}_{3}$ (162 mg, $1.0 \mathrm{mmol}$, 5.0 equiv). Then the mixture was stirred at $80{ }^{\circ} \mathrm{C}$ for $5 \mathrm{~h}$. After completion of the reaction, the mixture was quenched by adding $10 \mathrm{~mL}$ of $\mathrm{H}_{2} \mathrm{O}$ and extracted with DCM $(3 \times 10 \mathrm{~mL})$. The combined organic phase was washed with $\mathrm{H}_{2} \mathrm{O}(3 \times 10 \mathrm{~mL})$, dried over anhydrous $\mathrm{Na}_{2} \mathrm{SO}_{4}$, concentrated in vacuo and purified by flash silica gel chromatography to afford 6 (50.9 mg, over all yield: 66\%) as a red solid; m.p. $277-279{ }^{\circ} \mathrm{C}$ (Petroleum ether/EtOAc); R $f=0.30$ (Petroleum ether/EtOAc $=3 / 1) ;{ }^{1} \mathrm{H}$ NMR $\left(400 \mathrm{MHz}, \mathrm{DMSO}-d_{6}\right)$ : $\delta 9.89$ (s, 1H), 7.89-7.87 (m, 1H), 7.57-7.51 (m, 6H), 7.37 (d, $J=7.6 \mathrm{~Hz}, 2 \mathrm{H}), 7.30-7.21$ (m, 6H), 7.15-7.08 (m, 2H), 7.01-7.00 (m, 1H); ${ }^{13} \mathrm{C}$ NMR (100 MHz, DMSO-d $\left.d_{6}\right): \delta$ 166.1, 148.0, 143.6, 142.4, 140.0, 139.5, 132.8, 132.1, 132.0, 129.1, 128.8, 128.7, 128.6, 128.4, 128.3, 128.2, 127.7, 127.2, 127.0, 125.4, 124.8, 123.9, 120.9, 70.7; HRMS (ES ${ }^{+}$TOF) calcd for $\mathrm{C}_{28} \mathrm{H}_{20} \mathrm{NO}\left([\mathrm{M}+\mathrm{H}]^{+}\right)$: 386.1539, found 386.1541.

\section{Synthesis of compound 7:}

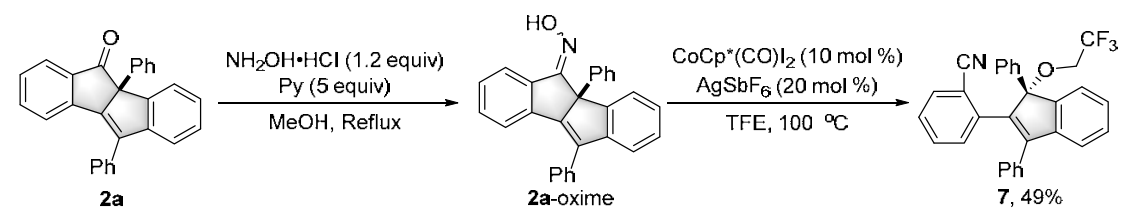

\section{2-(1,3-diphenyl-1-(2,2,2-trifluoroethoxy)-1H-inden-2-yl)benzonitrile (7)}

The solution of 4b,10-diphenylindeno[2,1-a]inden-5(4bH)-one 2a (74 mg, 0.2 mmol, 1.0 equiv) in 5 $\mathrm{mL}$ of $\mathrm{MeOH}$ was added pyridine (79 $\mathrm{mg}, 1.0 \mathrm{mmol}$, 5.0 equiv) and hydroxylamine hydrochloride (18 mg, $0.24 \mathrm{mmol}, 1.2$ equiv). Then the vessel was sealed and submerged in an oil bath preheated to 
$80{ }^{\circ} \mathrm{C}$ for $24 \mathrm{~h}$. After completion of the reaction, the solvent was removed under reduced pressure. 10 $\mathrm{mL}$ of $\mathrm{H}_{2} \mathrm{O}$ was added to the residual solid and extracted with $\mathrm{DCM}(3 \times 10 \mathrm{~mL})$. The combined organic layers were dried over $\mathrm{Na}_{2} \mathrm{SO}_{4}$ and concentrated in vacuo. The residue was purified with flash silica gel chromatography to afford 2a-oxime as a solid, which was used for next step. To a solution of 2a-oxime in $6 \mathrm{~mL}$ of TFE were added CoCp*(CO) $\mathrm{I}_{2}(9.5 \mathrm{mg}, 0.02 \mathrm{mmol}, 10 \mathrm{~mol} \%)$ and AgSbF $_{6}$ (13.7 mg, $0.04 \mathrm{mmol}, 20 \mathrm{~mol} \%$ ). Then the vessel was sealed and submerged in an oil bath preheated to $100{ }^{\circ} \mathrm{C}$ for $4 \mathrm{~h}$. After completion of the reaction, the mixture was quenched by adding 10 mL of $\mathrm{H}_{2} \mathrm{O}$ and extracted with DCM $(3 \times 10 \mathrm{~mL})$. The combined organic phase was washed with $\mathrm{H}_{2} \mathrm{O}$ $(3 \times 10 \mathrm{~mL})$, dried over anhydrous $\mathrm{Na}_{2} \mathrm{SO}_{4}$, concentrated in vacuo and purified by flash silica gel chromatography to afford 7 (46.2 mg, over all yield: $49 \%$ ) as a colorless liquid; $\mathrm{R} f=0.45$ (Petroleum ether/EtOAc = 10/1); ${ }^{1} \mathrm{H}$ NMR $\left(400 \mathrm{MHz}, \mathrm{CDCl}_{3}\right): \delta 7.52(\mathrm{~d}, J=7.6 \mathrm{~Hz}, 1 \mathrm{H}), 7.42-7.33(\mathrm{~m}, 11 \mathrm{H})$, 7.29-7.26 (m, 4H), 7.23-7.21 (m,1H), $6.99(\mathrm{~s}, 1 \mathrm{H}), 4.01-3.92(\mathrm{~m}, 1 \mathrm{H}), 3.75-3.66(\mathrm{~m}, 1 \mathrm{H}) ;{ }^{13} \mathrm{C}$ NMR (100 MHz, $\left.\mathrm{CDCl}_{3}\right): \delta 146.9,145.5,142.8,140.4,138.5,138.1,133.2,132.7,132.1,132.0,129.6$, 129.5, 128.6, 128.4,. 128.1, 127.9, 127.8, 127.2, 126.9, 125.9, 124.6 (q, $J=275.6 \mathrm{~Hz}), 122.1$, 118.2, 113.6, 92.7, 62.3 (q, $J=34.3 \mathrm{~Hz}$ ); HRMS (ES $\left.{ }^{+}-\mathrm{TOF}\right)$ calcd for $\mathrm{C}_{30} \mathrm{H}_{20} \mathrm{~F}_{3} \mathrm{NNaO}\left([\mathrm{M}+\mathrm{Na}]^{+}\right): 490.1389$, found 490.1399 .

\section{Synthesis of compound 8:}

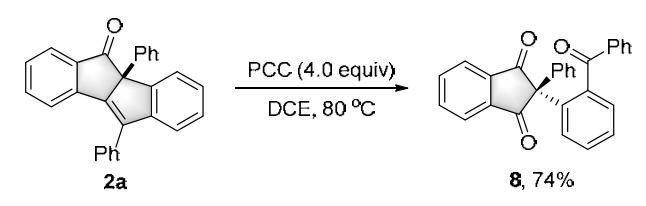

\section{2-(2-benzoylphenyl)-2-phenyl-1H-indene-1,3(2H)-dione (8)}

The solution of 4b,10-diphenylindeno[2,1-a]inden-5(4bH)-one 2a (74.4 mg, $0.2 \mathrm{mmol}, 1.0$ equiv) in $4 \mathrm{~mL}$ of DCE was added PCC (172 mg, $0.8 \mathrm{mmol}, 4.0$ equiv). Then the mixture was stirred at $80{ }^{\circ} \mathrm{C}$ 
for $4 \mathrm{~h}$. After completion of the reaction, the mixture was quenched by adding $10 \mathrm{~mL}$ of $\mathrm{H}_{2} \mathrm{O}$ and extracted with EtOAc $(3 \times 10 \mathrm{~mL})$. The combined organic phase was washed with $\mathrm{H}_{2} \mathrm{O}(3 \times 10 \mathrm{~mL})$, dried over anhydrous $\mathrm{Na}_{2} \mathrm{SO}_{4}$, concentrated in vacuo and purified by flash silica gel chromatography to afford 8 (59.8 mg, 74\%) as a white solid; m.p. 220-222 ${ }^{\circ} \mathrm{C}$ (Petroleum ether/EtOAc); Rf $=0.35$ $($ Petroleum ether/EtOAc $=5 / 1) ;{ }^{1} \mathrm{H}$ NMR $\left(400 \mathrm{MHz}, \mathrm{CDCl}_{3}\right): \delta$ 7.92-7.90 (m, 2H), 7.75-7.73 (m, 4H), 7.56-7.54 (m, 1H), 7.46-7.38 (m, 5H), 7.38-7.29 (m, 5H), 6.93 (d, $J=8.0 \mathrm{~Hz}, 1 \mathrm{H})$; ${ }^{13} \mathrm{C}$ NMR (100 MHz, $\left.\mathrm{CDCl}_{3}\right): \delta$ 198.6, 198.4, 140.9, 138.5, 137.3, 137.1, 136.6, 135.2, 134.2, 132.8, 131.3, 130.6, 130.4, 129.2, 129.1, 128.3, 128.1, 126.7, 123.5, 69.3; HRMS (ES ${ }^{+}$-TOF) calcd for $\mathrm{C}_{28} \mathrm{H}_{19} \mathrm{O}_{3}$ $\left([\mathrm{M}+\mathrm{H}]^{+}\right):$403.1329, found 403.1338 .

\section{Synthesis of compound 9 and its hydrolysis:}
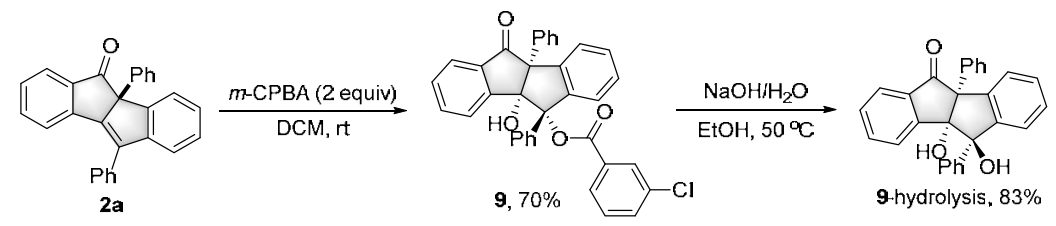

(4bR,5R,9bR)-4b-hydroxy-10-oxo-5,9b-diphenyl-4b,5,5a,9a,9b,10-hexahydroindeno[2,1-a]inden-5 -yl 3-chlorobenzoate (9)

The solution of 4b,10-diphenylindeno[2,1-a]inden-5(4bH)-one 2a (72.4 mg, 0.2 mmol, 1.0 equiv) in $2 \mathrm{~mL}$ of DCM was added $\mathrm{m}$-CPBA (64.8 $\mathrm{mg}, 0.4 \mathrm{mmol}$, 2.0 equiv) in the open air. Then the mixture was stirred at $25{ }^{\circ} \mathrm{C}$ for $48 \mathrm{~h}$. After completion of the reaction, the mixture was quenched by adding $10 \mathrm{~mL}$ of $\mathrm{H}_{2} \mathrm{O}$ and extracted with EtOAc $(3 \times 10 \mathrm{~mL})$. The combined organic phase was washed with $\mathrm{H}_{2} \mathrm{O}(3 \times 10 \mathrm{~mL})$, dried over anhydrous $\mathrm{Na}_{2} \mathrm{SO}_{4}$, concentrated in vacuo and purified by flash silica gel chromatography to afford 9 (76.0 $\mathrm{mg}, 70 \%$ ) as a white solid; m.p. $148-150{ }^{\circ} \mathrm{C}$ (Petroleum ether/EtOAc); $\mathrm{R} f=0.30$ (Petroleum ether/EtOAc $=5 / 1) ;{ }^{1} \mathrm{H}$ NMR $\left(400 \mathrm{MHz}, \mathrm{CDCl}_{3}\right): \delta$ 7.93-7.91 (m, 1H), 7.74-7.70 (m, 1H), 7.61-7.58 (m, 1H), 7.51-7.29 (m, 13H), 7.22-7.15 (m, 2H), 7.03 (d, $J=$ 
$7.6 \mathrm{~Hz}, 2 \mathrm{H}), 6.93(\mathrm{~s}, 1 \mathrm{H}), 5.74(\mathrm{~d}, J=8.0 \mathrm{~Hz}, 1 \mathrm{H}), 3.27$ (s, $1 \mathrm{H}) ;{ }^{13} \mathrm{C}$ NMR $\left(100 \mathrm{MHz}, \mathrm{CDCl}_{3}\right): \delta$ 203.0, 162.1, 150.0, 143.8, 138.6, 138.3, 136.0, 135.8, 134.5, 134.1, 133.2, 131.6, 131.4, 130.9, 130.5, 130.1, 129.5, 128.8, 128.7, 128.6, 128.5, 128.4, 128.1, 127.8, 126.5, 123.7, 93.3, 91.3, 73.0; HRMS (ES ${ }^{+}$TOF) calcd for $\mathrm{C}_{35} \mathrm{H}_{2} 4 \mathrm{ClO}_{4}\left([\mathrm{M}+\mathrm{H}]^{+}\right)$: 543.1358, found 543.1364.

(4bS,9bR,10R)-9b,10-dihydroxy-4b,10-diphenyl-9b,10-dihydroindeno[2,1-a]inden-5(4bH)-one (9-hydrolysis)

The solution of (4bR,5R,9bR)-4b-hydroxy-10-oxo-5,9b-diphenyl-4b,5,9b,10-tetrahydroindeno[2,1-a] inden-5-yl 3-chlorobenzoate 9 (0.1 mmol, $54.3 \mathrm{mg}, 1.0$ equiv) in $2 \mathrm{~mL}$ of EtOH was added $\mathrm{NaOH}$ (1 M solution in $\mathrm{H}_{2} \mathrm{O}, 1 \mathrm{~mL}$ ) with stiring at $50{ }^{\circ} \mathrm{C}$ for $4 \mathrm{~h}$. After completion of the reaction, the mixture was quenched by adding $10 \mathrm{~mL}$ of $\mathrm{H}_{2} \mathrm{O}$ and extracted with EtOAc $(3 \times 10 \mathrm{~mL})$. The combined organic phase was washed with $\mathrm{H}_{2} \mathrm{O}(3 \times 10 \mathrm{~mL})$, dried over anhydrous $\mathrm{Na}_{2} \mathrm{SO}_{4}$, concentrated in vacuo and purified by flash silica gel chromatography to afford 9-hydrolysis. (33.8 $\mathrm{mg}, 83 \%$ ) as a white solid; m.p. $170-172{ }^{\circ} \mathrm{C}$ (Petroleum ether/EtOAc); $\mathrm{R} f=0.30$ (Petroleum ether/EtOAc $\left.=3 / 1\right) ;{ }^{1} \mathrm{H}$ NMR $(400$ MHz, DMSO-d $\left.d_{6}\right): \delta 7.60(\mathrm{~d}, J=7.6 \mathrm{~Hz}, 1 \mathrm{H}), 7.43-7.16(\mathrm{~m}, 12 \mathrm{H}), 6.62-6.90(\mathrm{~m}, 2 \mathrm{H}), 6.72(\mathrm{~s}, 1 \mathrm{H})$, 5.95 (d, $J=7.6 \mathrm{~Hz}, 1 \mathrm{H}), 5.09$ (s, $1 \mathrm{H}) ;{ }^{13} \mathrm{C}$ NMR (100 MHz, DMSO-d 6 ): $\delta$ 204.2, 153.0, 145.2, 142.7, $141.4,140.5,135.2,134.0,130.3,129.6,129.2,128.9,128.3,128.1,127.6,127.3,126.6,126.5$, 125.9, 122.5, 90.8, 84.3, 73.4; HRMS (ES ${ }^{+}$TOF) calcd for $\mathrm{C}_{28} \mathrm{H}_{20} \mathrm{NaO}_{3}\left([\mathrm{M}+\mathrm{Na}]^{+}\right): 427.1305$, found 427.1314.

Synthesis of compound 10:

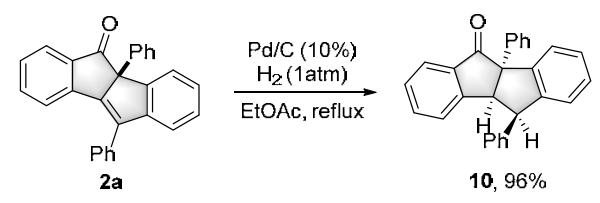

(4bS,9bS,10R)-4b,10-diphenyl-9b,10-dihydroindeno[2,1-a]inden-5(4bH)-one 
The solution of 4b,10-diphenylindeno[2,1-a]inden-5(4bH)-one 2a (74.4 mg, $0.2 \mathrm{mmol}, 1.0$ equiv) in $5 \mathrm{~mL}$ of EA was added Pd/C (10\%, $7.4 \mathrm{mg})$ with refluxing for $12 \mathrm{~h}$ under a hydrogen atmosphere. After completion of the reaction, the mixture was quenched by adding $10 \mathrm{~mL}$ of $\mathrm{H}_{2} \mathrm{O}$ and extracted with EtOAc $(3 \times 10 \mathrm{~mL})$. The combined organic phase was washed with $\mathrm{H}_{2} \mathrm{O}(3 \times 10 \mathrm{~mL})$, dried over anhydrous $\mathrm{Na}_{2} \mathrm{SO}_{4}$, concentrated in vacuo and purified by flash silica gel chromatography to afford 10 (71.4 mg, 96\%) a white solid; m.p. $184-187^{\circ} \mathrm{C}$ (Petroleum ether/EtOAc); $\mathrm{R} f=0.45$ (Petroleum ether/EtOAc = 10/1); ${ }^{1} \mathrm{H}$ NMR (400 MHz, $\left.\mathrm{CDCl}_{3}\right): \delta 7.76(\mathrm{~d}, J=7.2 \mathrm{~Hz}, 1 \mathrm{H}), 7.65-7.63(\mathrm{~m}, 1 \mathrm{H})$, 7.32-7.24 (m, 11H), 7.18-7.13 (m, 1H), 7.09-7.07 (m, 2H), 7.04-7.02 (m, 1H), 6.01 (d, J = 7.6 Hz, 1H), 5.18 (d, $J=9.6 \mathrm{~Hz}, 1 \mathrm{H}), 4.32$ (d, $J=9.6 \mathrm{~Hz}, 1 \mathrm{H}) ;{ }^{13} \mathrm{C}$ NMR $\left(100 \mathrm{MHz}, \mathrm{CDCl}_{3}\right): \delta$ 205.7, 152.2, 145.3, 143.0, 142.4, 139.4, 136.7, 133.7, 130.4, 128.8, 128.6, 128.5, 128.1, 127.8, 127.7, 127.5, 127.2, 127.0, 126.3, 125.9, 124.0, 70.8, 60.2, 53.0; HRMS (ES $-\mathrm{TOF})$ calcd for $\mathrm{C}_{28} \mathrm{H}_{21} \mathrm{O}\left([\mathrm{M}+\mathrm{H}]^{+}\right)$: 373.1587, found 373.1593.

\section{Emission spectra and density functional theory (DFT) bandgap calculation}

The cyclopenta-fused skeletons of compounds $\mathbf{2}$ and $\mathbf{3}$ might be intriguing units for new functional materials, including organic light emitting diodes, electroluminescence materials, and organic transistors. As a consequence, the fluorescent spectroscopic properties of a series of compounds 2 and 4 were checked as showed. Fluorescence spectra of $\mathbf{2}$ in both dilute dichloromethane (DCM) solution and solid state were measured (Figure $\boldsymbol{S} 1$ and $\boldsymbol{S} 2)$. All of tested compounds $\mathbf{2}$ covered the light ranging from 400 to $650 \mathrm{~nm}$ with a maximum at around $490 \mathrm{~nm}$ in its DCM solution. Obviously, the nature of substituents attaching to the carbonyl aromatic ring greatly affected the fluorescent intensity (See Figure S1: examples 2b, 2d, 2e and 2h). For examples, 2d with strong 
electron-donating group 7,9-diMeO displayed highest fluorescent intensity while $\mathbf{2 h}$ with strong electron-withdrawing group 8- $\mathrm{CF}_{3}$ showed the lowest one. The substituents on the benzene ring at the 4-position of compounds 2 had little effect on the fluorescence (See Figure S1: examples 2a, 2k and 2l). Functional groups tethered to indene units remarkably changed the fluorescent emission bands (See Figure S1: examples 2a, 2q, 2r and 2s). Luminescence wavelength of compound 2q (about $510 \mathrm{~nm}$ ) had a remarkable bathochromic shift together with increasing fluorescent intensity comparing with substrates $2 \mathbf{a}, 2 \mathbf{r}$ and $2 \mathbf{s}$ because of its donor-acceptor architecture. Moreover, all of the employed compounds also showed fluorescent emission in its solid state (Figure S2). Interestingly, compounds $2 \mathbf{i}$ and 2 s exhibited highly emissive in solid state probably due to the influence of S-atom in $\pi$-conjugated system in the case of $\mathbf{2} \mathbf{i}$ and the rotation restriction of the phenyl ring in the C11-position of 2s, respectively. Although no fluorescence of compounds 4 was observed in solid state due to the planar structure of $\mathbf{4}$ with the existence of intermolecular $\pi-\pi$ stacking, these compounds were strongly luminescent in dilute DCM, with maximum emissions at $410 \mathrm{~nm}$ and 430 nm, respectively (Figure S3). The substituents affected the $\pi$-conjugated systems in similar fashions. For examples, the strong electron-donating group on the aromatic ring (4e, $\mathbf{4 f}$, and $\mathbf{4 h})$ could pronouncedly increase fluorescent intensity and the electron-withdrawing group (4b and $\mathbf{4 c}$ ) led to decrease of fluorescent intensity. Furthermore, cuvette images of the compounds $\mathbf{2 b}, \mathbf{2 d}, \mathbf{2} \mathbf{j}, \mathbf{2 q}, \mathbf{4 e}$ and $\mathbf{4 h}$ were taken under a handheld UV-lamp ( $\left.\lambda_{\mathrm{ex}}=365 \mathrm{~nm}\right), \mathbf{2 b}, \mathbf{2 j}, \mathbf{4 e}, \mathbf{4 h}$ showed blue color and 2d, 2q displayed light green color (Figure S4). Besides, the fluorescent quantum yield of selected compounds $2 \mathbf{d}$ and $2 \mathbf{h}$ was found to be $\Phi_{2 \mathrm{~d}}=0.45$ and $\Phi_{4 \mathrm{~h}}=0.43$ based on the standard of quinine sulfate ( $\Phi=0.55$ in $0.1 \mathrm{M} \mathrm{H}_{2} \mathrm{SO}_{4}$, See Figure S5). Finally, the density functional theory (DFT) bandgap calculation shows that the HOMO-LUMO gap of compounds $\mathbf{2 b}, \mathbf{2 d}, \mathbf{2 h}, \mathbf{2 k}, \mathbf{2} \mathbf{l}$ and $\mathbf{2 q}$ 
decreases (see Figure S6) with the increase of push-pull electron effect (see Figure S1), which is consistent with their fluorescence response.

\section{Emission spectra:}

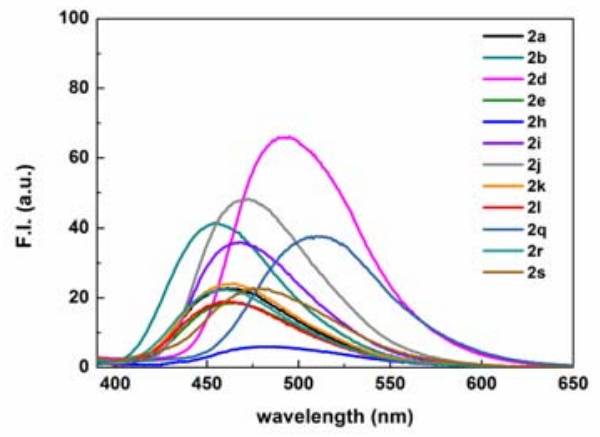

Figure S1. Emission spectra of $\mathbf{2 a}-\mathbf{b}, \mathbf{2 d}-\mathbf{e}, \mathbf{2 h}-\mathbf{l}$, and $\mathbf{2 q}-\mathbf{s}$ in DCM (c $\left.=10^{-5} \mathrm{~mol} / \mathrm{L}\right)$.

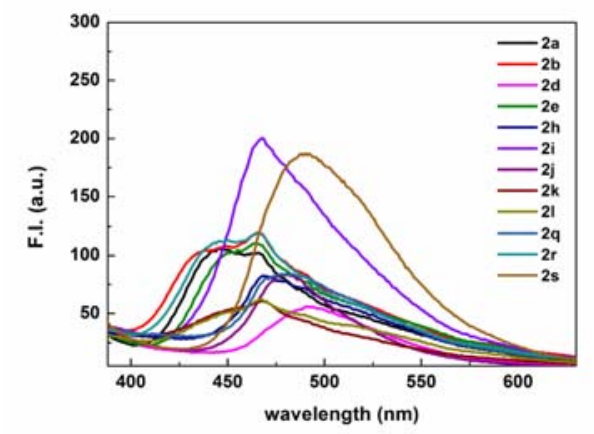

Figure S2. Solid emission spectra of $\mathbf{2 a}-\mathbf{b}, \mathbf{2 d}-\mathbf{e}, \mathbf{2 h}-\mathbf{l}$, and $\mathbf{2 q}-\mathbf{s}$

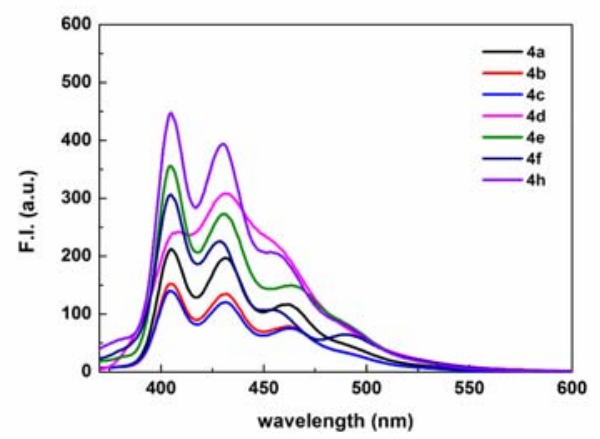

Figure S3. Emission spectra of $\mathbf{4 a}-\mathbf{f}$, and $\mathbf{4 h}$ in DCM $\left(\mathrm{c}=10^{-5} \mathrm{~mol} / \mathrm{L}\right)$ 


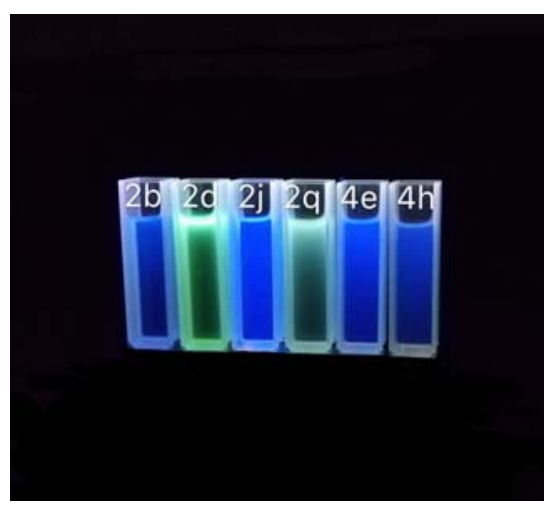

Figure S4. Cuvette images of the compounds $\mathbf{2 b}, \mathbf{2 d}, \mathbf{2 j}, \mathbf{2 q}, \mathbf{4 e}$ and $\mathbf{4 h}$ taken under a handheld UV-lamp $\left(\lambda_{\text {ex }}=365 \mathrm{~nm}\right)$.

Table S1. Emission and excitation wavelength of all compounds in DCM

\begin{tabular}{cccc}
\hline Compounds & $\begin{array}{c}\text { Emission } \\
\text { wavelength/nm }\end{array}$ & $\begin{array}{c}\text { Excitation } \\
\text { wavelength/nm }\end{array}$ & $\begin{array}{c}\text { Stokes } \\
\text { Shift/nm }\end{array}$ \\
\hline $\mathbf{2 a}$ & 384 & 463 & 79 \\
$\mathbf{2 b}$ & 395 & 455 & 60 \\
$\mathbf{2 d}$ & 401 & 492 & 91 \\
$\mathbf{2 e}$ & 351 & 464 & 113 \\
$\mathbf{2 h}$ & 410 & 484 & 74 \\
$\mathbf{2 i}$ & 387 & 467 & 80 \\
$\mathbf{2 j}$ & 393 & 473 & 80 \\
$\mathbf{2 k}$ & 324 & 464 & 140 \\
$\mathbf{2 l}$ & 355 & 461 & 106 \\
$\mathbf{2 q}$ & 363 & 511 & 148 \\
$\mathbf{2 r}$ & 341 & 458 & 117 \\
$\mathbf{2 s}$ & 414 & 480 & 66 \\
& & & \\
$\mathbf{4 a}$ & 315 & 431 & 116 \\
$\mathbf{4 b}$ & 318 & 432 & 114 \\
$\mathbf{4 c}$ & 320 & 433 & 113 \\
$\mathbf{4 d}$ & 340 & 432 & 92 \\
$\mathbf{4 e}$ & 337 & 430 & 93 \\
$\mathbf{4 f}$ & 326 & 428 & 102 \\
$\mathbf{4 h}$ & 316 & 430 & 114 \\
\hline
\end{tabular}

\section{Sample preparation:}

2d UV Absorbance 0.022 in DCM

4h UV Absorbance 0.033 in DCM 
Quinine sulfate UV Absorbance 0.024 in $0.1 \mathrm{M} \mathrm{H}_{2} \mathrm{SO}_{4}$

Quantum yield determination: The quantum yields of samples were determined based on quinine sulfate $0.1 \mathrm{M} \mathrm{H}_{2} \mathrm{SO}_{4}$ in deionized water.

$\left(\Phi_{2 \mathrm{~d}}=0.458253\right)$.

$\left(\Phi_{4 \mathrm{~h}}=0.433607\right)$.
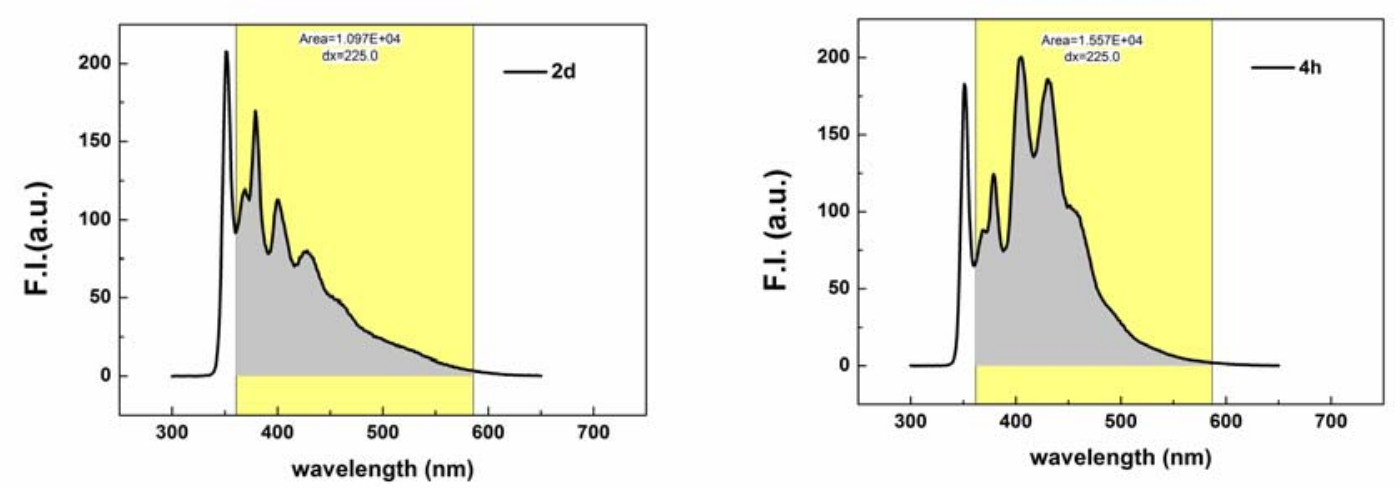

Figure S5. UV/FL spectra and quantum yields of $2 \mathrm{~d}$ and $4 \mathrm{~h}$ :

DFT calculations at the B3LYP-D3/6-311G(d,p) level of theory were carried out to optimize the structure and for the calculation of vibrational frequencies. DFT calculations are based on a Gaussian 09 program.

Density functional theory (DFT) calculation:
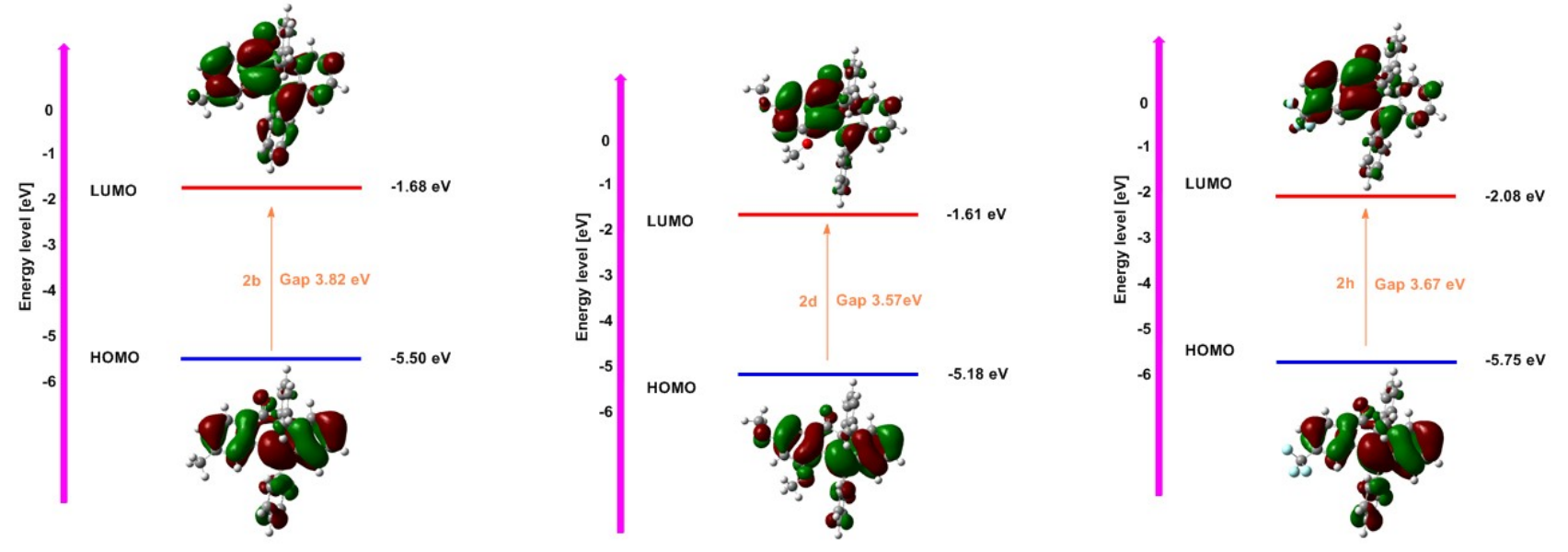

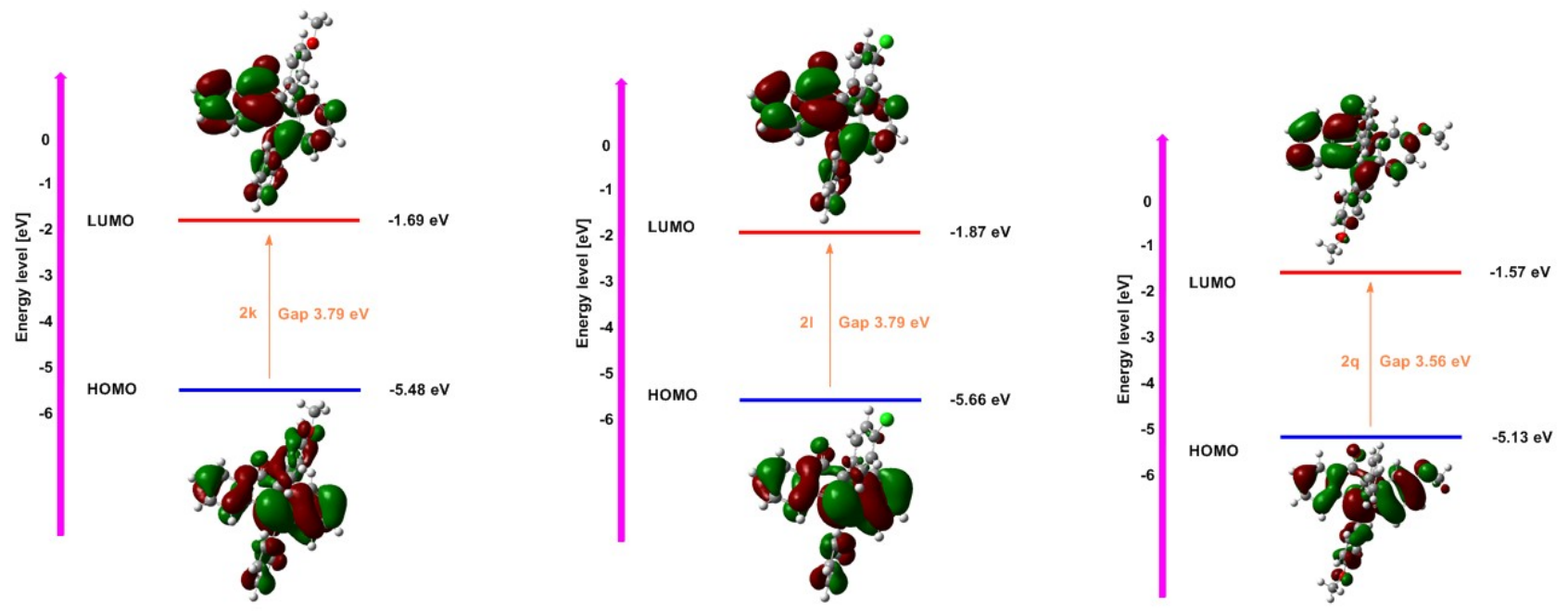

Figure S6. Energy gaps $(\Delta E \mathrm{~L} / \mathrm{H}), \mathrm{HOMO}$ and LUMO energy levels of the frontier molecular orbitals of compounds $\mathbf{2 b}, \mathbf{2 d}, \mathbf{2 h}, \mathbf{2 k}, \mathbf{2 l}$ and $\mathbf{2 q}$

\section{Cartesian coordinates for all relevant optimized structures}

\section{Structure 2b}

$\mathrm{E}(\mathrm{RPBE} 1 \mathrm{PBE})=-229.85041052554658$ a.u.

$\begin{array}{lrrr}01 & & & \\ \text { C } & -0.97384700 & 4.07157000 & -1.29267300 \\ \text { C } & -1.89413100 & 3.54881000 & -0.36180500 \\ \text { C } & -1.70659200 & 2.25905500 & 0.15243300 \\ \text { C } & -0.60154900 & 1.50420300 & -0.25143800 \\ \text { C } & 0.30995200 & 2.05404300 & -1.18397800 \\ \text { C } & 0.12593400 & 3.32957300 & -1.71271900 \\ \text { C } & -0.18633200 & 0.12528800 & 0.01061500 \\ \text { C } & 1.26184400 & -0.04343600 & -0.43421000 \\ \text { C } & 1.37815800 & 1.07608800 & -1.50861800 \\ \text { C } & -0.83314500 & -1.07560000 & 0.06342200 \\ \text { C } & 0.10481900 & -2.11801000 & -0.40912600 \\ \text { C } & 1.32098100 & -1.51209600 & -0.80172600 \\ \text { O } & 2.20315600 & 1.15050700 & -2.39911100 \\ \text { C } & -0.06252600 & -3.49914300 & -0.53579000 \\ \text { C } & 0.97897100 & -4.25657700 & -1.07971400 \\ \text { C } & 2.17350400 & -3.64967600 & -1.47763400 \\ \text { C } & 2.35934400 & -2.26982600 & -1.32235800 \\ \text { C } & 2.30097600 & 0.26960900 & 0.67108400 \\ \text { C } & -2.25459100 & -1.30725900 & 0.38085200 \\ \text { C } & -2.82591400 & -0.75659900 & 1.54116000\end{array}$




$\begin{array}{lrrc}\text { C } & -4.17156200 & -0.96191000 & 1.84404600 \\ \mathrm{C} & -4.97284500 & -1.72462700 & 0.99338500 \\ \mathrm{C} & -4.41770800 & -2.28265400 & -0.16022400 \\ \mathrm{C} & -3.07205900 & -2.08198000 & -0.46181400 \\ \mathrm{C} & 1.97586000 & 0.12340400 & 2.02571000 \\ \mathrm{C} & 2.93293900 & 0.34837600 & 3.01471800 \\ \mathrm{C} & 4.23115700 & 0.72356500 & 2.66633600 \\ \mathrm{C} & 4.56349800 & 0.86853000 & 1.31971500 \\ \mathrm{C} & 3.60710900 & 0.64402000 & 0.32813800 \\ \mathrm{C} & -3.06564200 & 4.38795700 & 0.08950800 \\ \mathrm{H} & -1.13473700 & 5.07073200 & -1.68881400 \\ \mathrm{H} & -2.42372800 & 1.85348700 & 0.85786400 \\ \mathrm{H} & 0.83021600 & 3.71964500 & -2.44121500 \\ \mathrm{H} & -0.98363000 & -3.97804800 & -0.21891700 \\ \mathrm{H} & 0.85887800 & -5.33040100 & -1.19047600 \\ \mathrm{H} & 2.96786100 & -4.25369700 & -1.90603800 \\ \mathrm{H} & 3.29348200 & -1.80092600 & -1.61409800 \\ \mathrm{H} & -2.19690400 & -0.18613100 & 2.21814800 \\ \mathrm{H} & -4.59132200 & -0.53328400 & 2.74958300 \\ \mathrm{H} & -6.02041400 & -1.88697000 & 1.22903300 \\ \mathrm{H} & -5.03563900 & -2.87378400 & -0.82992600 \\ \mathrm{H} & -2.65186500 & -2.50629000 & -1.36798600 \\ \mathrm{H} & 0.97062700 & -0.17279300 & 2.30603800 \\ \mathrm{H} & 2.66125800 & 0.23043200 & 4.05998300 \\ \mathrm{H} & 4.97536400 & 0.90169700 & 3.43707000 \\ \mathrm{H} & 5.57003800 & 1.16142500 & 1.03474400 \\ \mathrm{H} & 3.87258000 & 0.76976600 & -0.71610800 \\ \mathrm{H} & -3.52459600 & 4.91652800 & -0.75197200 \\ \mathrm{H} & -3.83510500 & 3.77766400 & 0.56934400 \\ & -2.74719900 & 5.14871000 & 0.81285500\end{array}$

\section{Structure 2d}

$\mathrm{E}(\mathrm{RPBE} 1 \mathrm{PBE})=-229.85041052554658$ a.u.

$\begin{array}{lrrr}01 & & & \\ \text { C } & 3.80986000 & -1.06160600 & 0.63928400 \\ \text { C } & 3.06698200 & -1.95801300 & -0.15688600 \\ \text { C } & 1.72748100 & -1.72144200 & -0.46279400 \\ \text { C } & 1.10623300 & -0.55368700 & 0.03170000 \\ \text { C } & 1.88507800 & 0.32620300 & 0.80813300 \\ \text { C } & 3.22119500 & 0.10125200 & 1.13646700 \\ \text { C } & -0.26400500 & -0.04148600 & -0.01334400\end{array}$




\begin{tabular}{|c|c|c|c|}
\hline C & -0.21624600 & 1.44092200 & 0.36070000 \\
\hline $\mathrm{C}$ & 1.06334300 & 1.49232000 & 1.23287200 \\
\hline $\mathrm{C}$ & -1.52348500 & -0.54349900 & 0.14460700 \\
\hline $\mathrm{C}$ & -2.37123900 & 0.52851100 & 0.71445300 \\
\hline $\mathrm{C}$ & -1.59143500 & 1.68408000 & 0.94325400 \\
\hline $\mathrm{O}$ & 1.36239100 & 2.33548700 & 2.05648000 \\
\hline $\mathrm{C}$ & -3.72266200 & 0.51387700 & 1.06697900 \\
\hline $\mathrm{C}$ & -4.27482700 & 1.65198700 & 1.66238200 \\
\hline $\mathrm{C}$ & -3.49590000 & 2.78921100 & 1.89335300 \\
\hline $\mathrm{C}$ & -2.14596200 & 2.81732700 & 1.51849600 \\
\hline $\mathrm{C}$ & 0.02283100 & 2.38109200 & -0.84742600 \\
\hline $\mathrm{C}$ & -2.02283900 & -1.92541200 & -0.04325000 \\
\hline $\mathrm{C}$ & -2.01977500 & -2.53251000 & -1.30888700 \\
\hline $\mathrm{C}$ & -2.53550400 & -3.81558700 & -1.48525600 \\
\hline $\mathrm{C}$ & -3.06268400 & -4.51786000 & -0.39942500 \\
\hline $\mathrm{C}$ & -3.07547000 & -3.92457400 & 0.86361300 \\
\hline $\mathrm{C}$ & -2.56837700 & -2.63697400 & 1.03853700 \\
\hline $\mathrm{C}$ & -0.31702900 & 1.99092100 & -2.14870500 \\
\hline $\mathrm{C}$ & -0.15484000 & 2.87069300 & -3.21881400 \\
\hline $\mathrm{C}$ & 0.35051400 & 4.15394600 & -3.00645200 \\
\hline $\mathrm{C}$ & 0.69149700 & 4.54988200 & -1.71326100 \\
\hline $\mathrm{C}$ & 0.53058500 & 3.67109100 & -0.64120000 \\
\hline $\mathrm{O}$ & 0.96935500 & -2.54057700 & -1.23402200 \\
\hline $\mathrm{C}$ & 1.53260800 & -3.75833700 & -1.70027400 \\
\hline $\mathrm{O}$ & 5.10087500 & -1.43545100 & 0.85848000 \\
\hline $\mathrm{C}$ & 5.91706700 & -0.58108700 & 1.64951200 \\
\hline $\mathrm{H}$ & 3.58316700 & -2.83689500 & -0.52150700 \\
\hline $\mathrm{H}$ & 3.74636400 & 0.81687400 & 1.75582200 \\
\hline $\mathrm{H}$ & -4.33118800 & -0.36711400 & 0.88858400 \\
\hline $\mathrm{H}$ & -5.32312600 & 1.65219900 & 1.94721100 \\
\hline $\mathrm{H}$ & -3.94075700 & 3.66072000 & 2.36455200 \\
\hline $\mathrm{H}$ & -1.54449200 & 3.70491600 & 1.68596800 \\
\hline $\mathrm{H}$ & -1.61012900 & -1.98888500 & -2.15315800 \\
\hline $\mathrm{H}$ & -2.53446400 & -4.26479200 & -2.47470700 \\
\hline $\mathrm{H}$ & -3.46566700 & -5.51697600 & -0.53806400 \\
\hline $\mathrm{H}$ & -3.48259500 & -4.46265400 & 1.71491400 \\
\hline $\mathrm{H}$ & -2.58221800 & -2.17879000 & 2.02294600 \\
\hline $\mathrm{H}$ & -0.71397900 & 0.99674700 & -2.32290000 \\
\hline $\mathrm{H}$ & -0.42442300 & 2.54987900 & -4.22118500 \\
\hline $\mathrm{H}$ & 0.47851300 & 4.83736300 & -3.84085100 \\
\hline $\mathrm{H}$ & 1.08835800 & 5.54516700 & -1.53411000 \\
\hline $\mathrm{H}$ & 0.81040000 & 3.98353600 & 0.35946100 \\
\hline $\mathrm{H}$ & 1.87312200 & -4.38688800 & -0.86866400 \\
\hline $\mathrm{H}$ & 2.37091500 & -3.57768100 & -2.38456300 \\
\hline
\end{tabular}




$\begin{array}{crrr}\mathrm{H} & 0.73021700 & -4.26969000 & -2.23258400 \\ \mathrm{H} & 6.89553700 & -1.06050300 & 1.69601700 \\ \mathrm{H} & 5.51775200 & -0.47099900 & 2.66508300 \\ \mathrm{H} & 6.01975300 & 0.41134900 & 1.19402200\end{array}$

\section{Structure 2h}

$\mathrm{E}(\mathrm{RPBE} 1 \mathrm{PBE})=-229.85041052554658$ a.u.

\begin{tabular}{|c|c|c|c|}
\hline C & 2.74256000 & -2.37097200 & -1.33185000 \\
\hline C & 3.09931700 & -1.37261100 & -0.41135800 \\
\hline C & 2.15690200 & -0.49006400 & 0.12167700 \\
\hline C & 0.82197100 & -0.61851000 & -0.27404400 \\
\hline C & 0.47190300 & -1.62621700 & -1.20405100 \\
\hline C & 1.41692400 & -2.49623300 & -1.73957900 \\
\hline C & -0.37890700 & 0.16759200 & 0.00566300 \\
\hline C & -1.60126100 & -0.62886200 & -0.43637500 \\
\hline C & -0.98331700 & -1.55526600 & -1.51956300 \\
\hline C & -0.65079400 & 1.50385000 & 0.06813200 \\
\hline C & -2.04351400 & 1.70484300 & -0.38791400 \\
\hline C & -2.59193200 & 0.46352100 & -0.78610000 \\
\hline $\mathrm{O}$ & -1.56637300 & -2.13503800 & -2.41337900 \\
\hline C & -2.80136400 & 2.87350600 & -0.49469900 \\
\hline C & -4.09121700 & 2.79229500 & -1.02671100 \\
\hline C & -4.62220600 & 1.56438100 & -1.43172800 \\
\hline C & -3.87954500 & 0.38447500 & -1.29515700 \\
\hline C & -2.18539000 & -1.55224000 & 0.66242900 \\
\hline C & 0.29609200 & 2.58911100 & 0.38325100 \\
\hline C & 1.10432200 & 2.52033400 & 1.53124000 \\
\hline C & 2.01275900 & 3.53558900 & 1.82874900 \\
\hline C & 2.12908800 & 4.64047800 & 0.98433000 \\
\hline C & 1.32833700 & 4.72502400 & -0.15662300 \\
\hline C & 0.41659700 & 3.71383700 & -0.45291900 \\
\hline C & -1.96926700 & -1.28652700 & 2.02025200 \\
\hline C & -2.54893500 & -2.09046700 & 3.00163200 \\
\hline C & -3.35300600 & -3.17251000 & 2.64168800 \\
\hline C & -3.57389300 & -3.44268900 & 1.29123800 \\
\hline C & -2.99416300 & -2.64043300 & 0.30756300 \\
\hline $\mathrm{H}$ & 3.50396800 & -3.04124600 & -1.71491600 \\
\hline $\mathrm{H}$ & 2.46457200 & 0.27009500 & 0.82791300 \\
\hline $\mathrm{H}$ & 1.11338200 & -3.25011900 & -2.45893100 \\
\hline $\mathrm{H}$ & -2.39792900 & 3.82775900 & -0.17142400 \\
\hline & -4.68899600 & 3.69396100 & -1.12250700 \\
\hline
\end{tabular}




$\begin{array}{lrrr}\mathrm{H} & -5.62320000 & 1.52281300 & -1.85066600 \\ \mathrm{H} & -4.30046000 & -0.57048900 & -1.59204500 \\ \mathrm{H} & 0.99397000 & 1.67544800 & 2.20463500 \\ \mathrm{H} & 2.62427500 & 3.46592400 & 2.72350100 \\ \mathrm{H} & 2.83604700 & 5.43172800 & 1.21491400 \\ \mathrm{H} & 1.41714800 & 5.57953400 & -0.82105100 \\ \mathrm{H} & -0.19028500 & 3.77924800 & -1.35024400\end{array}$

\section{Structure 2k}

$\mathrm{E}(\mathrm{RPBE} 1 \mathrm{PBE})=-229.85041052554658$ a.u.

$\begin{array}{lrrr}\text { O } 1 & & & \\ \text { C } & 0.76773700 & 4.45132400 & 1.29664500 \\ \text { C } & 1.55550600 & 4.12686700 & 0.18264100 \\ \text { C } & 1.60411600 & 2.82768100 & -0.32736400 \\ \text { C } & 0.83871400 & 1.83305200 & 0.29077100 \\ \text { C } & 0.04542500 & 2.17209600 & 1.41434600 \\ \text { C } & 0.00811500 & 3.46612400 & 1.92678600 \\ \text { C } & 0.72739600 & 0.38775200 & 0.09084500 \\ \text { C } & -0.50134800 & -0.11849500 & 0.83823200 \\ \text { C } & -0.65065800 & 0.96716700 & 1.94021400 \\ \text { C } & 1.62014800 & -0.61940000 & -0.13753700 \\ \text { C } & 1.09319900 & -1.84684500 & 0.49959600 \\ \text { C } & -0.11673300 & -1.54637200 & 1.16716400 \\ \text { O } & -1.25022000 & 0.86482500 & 2.99309100 \\ \text { C } & 1.61491900 & -3.14212900 & 0.54410300 \\ \text { C } & 0.93487000 & -4.11699300 & 1.27973500 \\ \text { C } & -0.25386300 & -3.81015600 & 1.94802800 \\ \text { C } & -0.79901300 & -2.52167400 & 1.87905100 \\ \text { C } & -1.80112600 & -0.08763300 & -0.00184800 \\ \text { C } & 2.94765600 & -0.50471600 & -0.76936900 \\ \text { C } & 4.09479900 & -1.04240100 & -0.15803400 \\ \text { C } & 5.34634900 & -0.91591300 & -0.75766800 \\ \text { C } & 5.47824400 & -0.26020200 & -1.98358500 \\ \text { C } & 4.34768500 & 0.27026700 & -2.60641100 \\ \text { C } & 3.09520300 & 0.14760100 & -2.00574100 \\ \text { C } & -1.76394900 & -0.18130100 & -1.40198800 \\ \text { C } & -2.93215600 & -0.21482300 & -2.15047000 \\ \text { C } & -4.18088300 & -0.15709800 & -1.51497800 \\ \text { C } & -4.23622900 & -0.06724000 & -0.12100500 \\ \text { C } & -3.05132900 & -0.03289600 & 0.61985800 \\ \text { O } & -5.26909300 & -0.19242900 & -2.33908600\end{array}$




$\begin{array}{lrrr}\mathrm{C} & -6.55800700 & -0.13977400 & -1.74858400 \\ \mathrm{H} & 0.75480800 & 5.47156700 & 1.66764900 \\ \mathrm{H} & 2.14382200 & 4.90479000 & -0.29587500 \\ \mathrm{H} & 2.22674400 & 2.59954200 & -1.18461200 \\ \mathrm{H} & -0.60018900 & 3.68342300 & 2.79942900 \\ \mathrm{H} & 2.53116400 & -3.39033300 & 0.01783200 \\ \mathrm{H} & 1.33363000 & -5.12616000 & 1.32840800 \\ \mathrm{H} & -0.76317900 & -4.57912800 & 2.52137300 \\ \mathrm{H} & -1.73170100 & -2.28775200 & 2.38208900 \\ \mathrm{H} & 4.00316600 & -1.53925300 & 0.80238700 \\ \mathrm{H} & 6.22214700 & -1.32714200 & -0.26407100 \\ \mathrm{H} & 6.45400000 & -0.16737700 & -2.45108200 \\ \mathrm{H} & 4.43835400 & 0.77237400 & -3.56530500 \\ \mathrm{H} & 2.21211800 & 0.53472200 & -2.50533800 \\ \mathrm{H} & -0.80734100 & -0.23293600 & -1.91104800 \\ \mathrm{H} & -2.90365200 & -0.28556100 & -3.23289600 \\ \mathrm{H} & -5.18504400 & -0.01869300 & 0.39987600 \\ \mathrm{H} & -3.11069000 & 0.04694700 & 1.70022800 \\ \mathrm{H} & -7.26984700 & -0.18183100 & -2.57425600 \\ \mathrm{H} & -6.73236800 & -0.99152800 & -1.07872200 \\ \mathrm{H} & -6.71015900 & 0.79194400 & -1.18871000\end{array}$

\section{Structure 2l}

$\mathrm{E}(\mathrm{RPBE} 1 \mathrm{PBE})=-229.85041052554658$ a.u.

$\begin{array}{lrrr}01 & & & \\ \mathrm{C} & 0.82721200 & 4.46501900 & 1.26563100 \\ \mathrm{C} & 1.58281800 & 4.11909500 & 0.13576700 \\ \mathrm{C} & 1.60105600 & 2.81485900 & -0.36310900 \\ \mathrm{C} & 0.83748100 & 1.83678200 & 0.28291900 \\ \mathrm{C} & 0.07728200 & 2.19727200 & 1.42291000 \\ \mathrm{C} & 0.06999700 & 3.49671000 & 1.92371700 \\ \mathrm{C} & 0.70288500 & 0.39115700 & 0.09878300 \\ \mathrm{C} & -0.51156100 & -0.09226600 & 0.88322200 \\ \mathrm{C} & -0.62447000 & 1.00967600 & 1.97585500 \\ \mathrm{C} & 1.57838400 & -0.62915800 & -0.13860700 \\ \mathrm{C} & 1.05357200 & -1.84273400 & 0.52599500 \\ \mathrm{C} & -0.13504000 & -1.52108600 & 1.22150400 \\ \mathrm{O} & -1.20846400 & 0.92530600 & 3.03867000 \\ \mathrm{C} & 1.56237700 & -3.14290000 & 0.57370000 \\ \mathrm{C} & 0.89142100 & -4.10084500 & 1.33937200 \\ \mathrm{C} & -0.27540700 & -3.77262100 & 2.03519700 \\ \mathrm{C} & -0.80858000 & -2.47909900 & 1.96437900\end{array}$




$\begin{array}{lrrc}\mathrm{C} & -1.83170400 & -0.06931600 & 0.07661400 \\ \mathrm{C} & 2.89239900 & -0.53627900 & -0.80136400 \\ \mathrm{C} & 4.04708900 & -1.07833700 & -0.20840900 \\ \mathrm{C} & 5.28642400 & -0.97087900 & -0.83639200 \\ \mathrm{C} & 5.39771000 & -0.33090300 & -2.07262300 \\ \mathrm{C} & 4.25913700 & 0.20328900 & -2.67738000 \\ \mathrm{C} & 3.01898400 & 0.10023400 & -2.04821000 \\ \mathrm{C} & -1.82756300 & -0.16214400 & -1.32040600 \\ \mathrm{C} & -3.01864900 & -0.20125200 & -2.04333000 \\ \mathrm{C} & -4.23152800 & -0.14715000 & -1.35967500 \\ \mathrm{C} & -4.26469200 & -0.05639400 & 0.02957100 \\ \mathrm{C} & -3.06489300 & -0.01764400 & 0.73968600 \\ \mathrm{Cl} & -5.74055800 & -0.19121600 & -2.26445400 \\ \mathrm{H} & 0.83716400 & 5.48875700 & 1.62669100 \\ \mathrm{H} & 2.16964400 & 4.88429400 & -0.36445600 \\ \mathrm{H} & 2.19913500 & 2.57032800 & -1.23315700 \\ \mathrm{H} & -0.51397600 & 3.73086300 & 2.80847400 \\ \mathrm{H} & 2.46200400 & -3.40744200 & 0.02725800 \\ \mathrm{H} & 1.28038600 & -5.11361000 & 1.39043400 \\ \mathrm{H} & -0.77737300 & -4.52836000 & 2.63190400 \\ \mathrm{H} & -1.72412300 & -2.22956200 & 2.49078900 \\ \mathrm{H} & 3.97182400 & -1.56278200 & 0.75972200 \\ \mathrm{H} & 6.16881000 & -1.38465800 & -0.35699700 \\ \mathrm{H} & 6.36389100 & -0.25307900 & -2.56211000 \\ \mathrm{H} & 4.33385900 & 0.69312400 & -3.64390100 \\ \mathrm{H} & 2.12924500 & 0.49072500 & -2.53319500 \\ \mathrm{H} & -0.88405400 & -0.20915400 & -1.85314400 \\ \mathrm{H} & -3.00585600 & -0.27238700 & -3.12523000 \\ \mathrm{H} & -5.21465800 & -0.01225600 & 0.55043000 \\ \mathrm{H} & -3.09156800 & 0.06490500 & 1.82096200\end{array}$

\section{Structure 2q}

$\mathrm{E}(\mathrm{RPBE} 1 \mathrm{PBE})=-229.85041052554658$ a.u.

$\begin{array}{lrrr}01 & & & \\ \mathrm{C} & 0.62575500 & -4.48274200 & -2.05956800 \\ \mathrm{C} & 1.68822100 & -4.03819700 & -1.25905800 \\ \mathrm{C} & 1.61409900 & -2.84508700 & -0.53746400 \\ \mathrm{C} & 0.44379000 & -2.08081300 & -0.61459900 \\ \mathrm{C} & -0.62317300 & -2.54028600 & -1.42674600 \\ \mathrm{C} & -0.54143700 & -3.72561500 & -2.15271800 \\ \mathrm{C} & 0.06985200 & -0.77005500 & -0.08581800\end{array}$




\begin{tabular}{|c|c|c|c|}
\hline C & -1.43807400 & -0.59400700 & -0.23510300 \\
\hline $\mathrm{C}$ & -1.74590000 & -1.56692100 & -1.40739200 \\
\hline $\mathrm{C}$ & 0.70303100 & 0.43737600 & 0.01318600 \\
\hline C & -0.31426300 & 1.49769700 & -0.13522700 \\
\hline C & -1.57831100 & 0.90806300 & -0.38780000 \\
\hline $\mathrm{O}$ & -2.72472800 & -1.55674500 & -2.12899100 \\
\hline C & -0.20327900 & 2.88603800 & -0.09543000 \\
\hline $\mathrm{C}$ & -1.33081300 & 3.67953900 & -0.33649200 \\
\hline $\mathrm{C}$ & -2.57041800 & 3.08131900 & -0.60259200 \\
\hline C & -2.70098000 & 1.67919900 & -0.60869000 \\
\hline C & -2.25429800 & -1.07901200 & 0.98900800 \\
\hline C & 2.15274100 & 0.67719200 & 0.10877900 \\
\hline C & 2.80166200 & 1.61445600 & -0.72130100 \\
\hline C & 4.16864100 & 1.82817100 & -0.63356100 \\
\hline C & 4.93837400 & 1.11747200 & 0.30076200 \\
\hline C & 4.31527100 & 0.18901300 & 1.14216800 \\
\hline C & 2.93847500 & -0.01908100 & 1.03797000 \\
\hline C & -1.67863300 & -1.12646300 & 2.26486100 \\
\hline C & -2.43591600 & -1.50601700 & 3.37287700 \\
\hline C & -3.78111000 & -1.84558800 & 3.22387300 \\
\hline $\mathrm{C}$ & -4.36203400 & -1.79991300 & 1.95665800 \\
\hline C & -3.60608200 & -1.42040100 & 0.84659900 \\
\hline $\mathrm{O}$ & 6.27077600 & 1.40598200 & 0.31130100 \\
\hline C & 7.10190500 & 0.71504500 & 1.23171300 \\
\hline $\mathrm{O}$ & -3.71899400 & 3.77127800 & -0.86191400 \\
\hline C & -3.66647800 & 5.18854700 & -0.88165400 \\
\hline $\mathrm{H}$ & 0.71757400 & -5.41554400 & -2.60739500 \\
\hline $\mathrm{H}$ & 2.59293500 & -4.63680500 & -1.19824200 \\
\hline $\mathrm{H}$ & 2.45210200 & -2.52030400 & 0.06818400 \\
\hline $\mathrm{H}$ & -1.37556900 & -4.03460200 & -2.77531700 \\
\hline $\mathrm{H}$ & 0.74835700 & 3.36128600 & 0.12078300 \\
\hline $\mathrm{H}$ & -1.23270400 & 4.75796800 & -0.31052000 \\
\hline $\mathrm{H}$ & -3.67518000 & 1.24183900 & -0.79553600 \\
\hline $\mathrm{H}$ & 2.22473200 & 2.16067100 & -1.46044600 \\
\hline $\mathrm{H}$ & 4.66980800 & 2.53885300 & -1.28238300 \\
\hline $\mathrm{H}$ & 4.88181000 & -0.36333800 & 1.88213900 \\
\hline $\mathrm{H}$ & 2.45790100 & -0.72008000 & 1.71393800 \\
\hline $\mathrm{H}$ & -0.63465600 & -0.85997800 & 2.39062100 \\
\hline $\mathrm{H}$ & -1.97118700 & -1.53655700 & 4.35446500 \\
\hline $\mathrm{H}$ & -4.36977600 & -2.14390200 & 4.08652500 \\
\hline $\mathrm{H}$ & -5.40767900 & -2.06389100 & 1.82604800 \\
\hline $\mathrm{H}$ & -4.06408300 & -1.39948400 & -0.13665100 \\
\hline $\mathrm{H}$ & 8.11237300 & 1.09016800 & 1.06448700 \\
\hline $\mathrm{H}$ & 6.81023400 & 0.91554400 & 2.27050700 \\
\hline
\end{tabular}




$\begin{array}{lrrc}\mathrm{H} & 7.08560000 & -0.36825200 & 1.05728100 \\ \mathrm{H} & -4.67722100 & 5.52354000 & -1.11886900 \\ \mathrm{H} & -3.37105700 & 5.59751500 & 0.09314500 \\ \mathrm{H} & -2.97444600 & 5.55764800 & -1.64939300\end{array}$

\section{Preparation of allenyl ketones 1}

Synthesis of starting materials $1 a-1 n, 1 p-1 y$ by revised method, and ${ }^{1} \mathrm{H}$ and ${ }^{13} \mathrm{C}$ NMR spectra data for the reported ones showed good agreement with the literature data ${ }^{4}$ (H. Ren, M. Miao, H. Xu, Y. Luo, M. Jin, Z. Chen, J. Xu, Synthesis 2017, 50, 349). Belows are new general procedure and the summarized characterization data for the newly synthesized allenyl ketones $\mathbf{1}$.

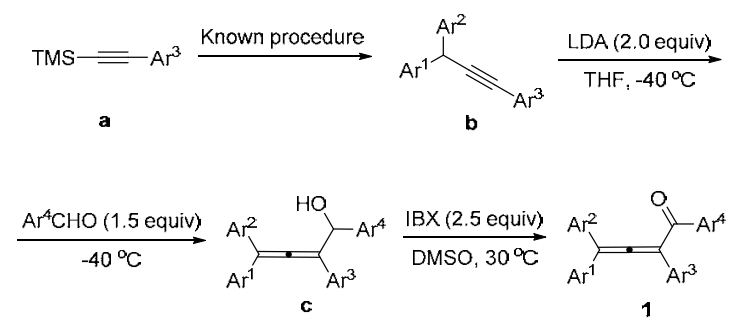

To a solution of 1,3,3-triarylpropyne $\mathbf{b}$, which was synthesized according to the reported procedure ${ }^{1}$ (5.0 mmol, 1.0 equiv) in $20 \mathrm{~mL}$ of anhydrous THF, was added dropwise to a solution of LDA (2 M in hexane, $10.0 \mathrm{mmol}, 2.0$ equiv) at $-40{ }^{\circ} \mathrm{C}$ under the $\mathrm{N}_{2}$ atmosphere. The reaction was stirred for 20 min. Then aldehyde (7.5 mmol, 1.5 equiv) dissolving in $5 \mathrm{~mL}$ of dry THF was injected to the mixture by a syringe at $-40{ }^{\circ} \mathrm{C}$. After $15 \mathrm{~min}$, the mixture was allowed to warm up to room temperature, quenched by adding $50 \mathrm{~mL}$ of $\mathrm{H}_{2} \mathrm{O}$ and extracted with $\mathrm{EA}(3 \times 50 \mathrm{~mL})$. The combined organic layers were dried over $\mathrm{Na}_{2} \mathrm{SO}_{4}$ and concentrated in vacuo. The residue was purified with flash silica gel chromatography to afford allenyl alcohol c. To a solution of the mixture of $\mathbf{c}$ in $20 \mathrm{~mL}$ of DMSO were added 2.5 equiv of IBX with stirring at $30{ }^{\circ} \mathrm{C}$ for $30 \mathrm{~min}$. After completion of the reaction, the mixture was quenched by adding $20 \mathrm{~mL}$ of $\mathrm{H}_{2} \mathrm{O}$ and extracted with EtOAc $(3 \times 50 \mathrm{~mL})$. The combined organic phase was washed with $\mathrm{H}_{2} \mathrm{O}(3 \times 50 \mathrm{~mL})$, dried over anhydrous $\mathrm{Na}_{2} \mathrm{SO}_{4}$, 
concentrated in vacuo and purified by flash silica gel chromatography to give the starting materials $\mathbf{1}$.

\section{1,2,4,4-tetraphenylbuta-2,3-dien-1-one (1a)}

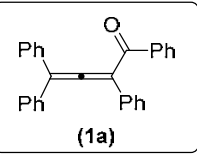

The reaction of prop-2-yne-1,1,3-triyltribenzene (1.342 g, $5.0 \mathrm{mmol}$, 1.0 equiv), LDA (5 mL, 2.0 M in hexane, 2.0 equiv), and benzaldehyde (0.795 g, $7.5 \mathrm{mmol}, 1.5$ equiv) in $20 \mathrm{~mL}$ of THF. The resulted mixture was further oxidized by IBX (3.500 g, 12.5 mmol, 2.5 equiv) in $20 \mathrm{~mL}$ of DMSO to afford 1a (1.562 g, 84\%) as a yellow solid. (Known compound)

\section{2,4,4-triphenyl-1-(p-tolyl)buta-2,3-dien-1-one (1b)}

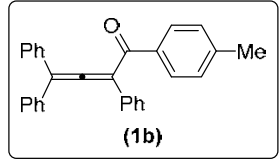

The reaction of prop-2-yne-1,1,3-triyltribenzene (1.342 g, $5.0 \mathrm{mmol}, 1.0$ equiv), LDA (5 mL, 2.0 M in hexane, 2.0 equiv), and 4-methylbenzaldehyde (0.900 g, 7.5 mmol, 1.5 equiv) in $20 \mathrm{~mL}$ of THF. The resulted mixture was further oxidized by IBX (3.500 g, 12.5 mmol, 2.5 equiv) in $20 \mathrm{~mL}$ of DMSO to afford $\mathbf{1 b}(1.585 \mathrm{~g}, 82 \%)$ as a yellow solid. (Known compound)

\section{1-(4-methoxyphenyl)-2,4,4-triphenylbuta-2,3-dien-1-one (1c)}

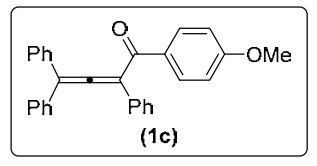

The reaction of prop-2-yne-1,1,3-triyltribenzene (1.340 g, 5.0 mmol, 1.0 equiv), LDA (5 mL, 2.0 M in hexane, 2.0 equiv), and 4-methoxybenzaldehyde (1.022 g, $7.5 \mathrm{mmol}, 1.5$ equiv) in $20 \mathrm{~mL}$ of THF. The resulted mixture was further oxidized by IBX (3.500g, $12.5 \mathrm{mmol}, 2.5$ equiv) in $20 \mathrm{~mL}$ of DMSO to afford 1c (1.610 g, 80\%) as a yellow solid. (Known compound)

\section{1-(3,5-dimethoxyphenyl)-2,4,4-triphenylbuta-2,3-dien-1-one (1d)}

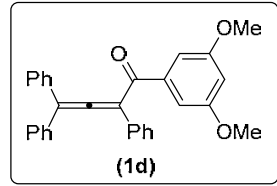

The reaction of prop-2-yne-1,1,3-triyltribenzene (1.342 g, $5.0 \mathrm{mmol}, 1.0$ equiv), LDA (5 mL, 2.0 M in hexane, 2.0 equiv), and 3,5-dimethoxybenzaldehyde (1.247 
g, $7.5 \mathrm{mmol}, 1.5$ equiv) in $20 \mathrm{~mL}$ of THF. The resulted mixture was further oxidized by IBX (3.500 g, 12.5 mmol, 2.5 equiv) in $20 \mathrm{~mL}$ of DMSO to afford $\mathbf{1 d}(1.758 \mathrm{~g}, 81 \%)$ as a yellow solid. (Known compound)

\section{1-(4-chlorophenyl)-2,4,4-triphenylbuta-2,3-dien-1-one (1e)}

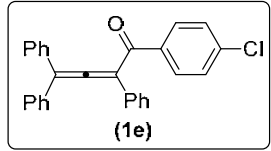

The reaction of prop-2-yne-1,1,3-triyltribenzene (1.342 g, $5.0 \mathrm{mmol}, 1.0$ equiv), LDA (5 mL, 2.0 M in hexane, 2.0 equiv), and 4-chlorobenzaldehyde (1.054 g, 7.5 mmol, 1.5 equiv) in $20 \mathrm{~mL}$ of THF. The resulted mixture was further oxidized by IBX (3.500 g, 12.5 mmol, 2.5 equiv) in $20 \mathrm{~mL}$ of DMSO to afford 1e (1.669 g, 82\%) as a yellow solid. (Known compound)

\section{1-(3-chlorophenyl)-2,4,4-triphenylbuta-2,3-dien-1-one (1f)}

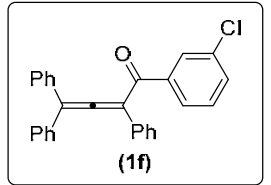

The reaction of prop-2-yne-1,1,3-triyltribenzene (1.342 g, $5.0 \mathrm{mmol}, 1.0$ equiv), LDA (5 mL, 2.0 M in hexane, 2.0 equiv), and 3-chlorobenzaldehyde (1.054 g, 7.5 mmol, 1.5 equiv) in $20 \mathrm{~mL}$ of THF. The resulted mixture was further oxidized by IBX (3.500 g, 12.5 mmol, 2.5 equiv) in $20 \mathrm{~mL}$ of DMSO to afford $\mathbf{1 f}(1.664 \mathrm{~g}, 82 \%)$ as a yellow solid. (Known compound)

\section{1-(4-bromophenyl)-2,4,4-triphenylbuta-2,3-dien-1-one (1g)}

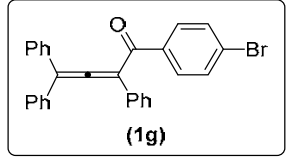

The reaction of prop-2-yne-1,1,3-triyltribenzene (1.342 g, 5.0 mmol, 1.0 equiv), LDA (5 mL, 2.0 M in hexane, 2.0 equiv), and 4-bromobenzaldehyde (1.388 g, 7.5 mmol, 1.5 equiv) in $20 \mathrm{~mL}$ of THF. The resulted mixture was further oxidized by IBX (3.500 g, 12.5 mmol, 2.5 equiv) in $20 \mathrm{~mL}$ of DMSO to afford $1 \mathrm{~g}(1.760 \mathrm{~g}, 78 \%)$ as a yellow solid. (Known compound) 


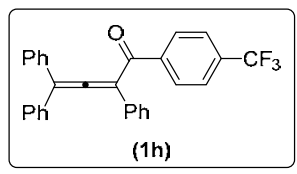

The reaction of prop-2-yne-1,1,3-triyltribenzene (1.342 g, $5.0 \mathrm{mmol}, 1.0$ equiv), LDA (5 mL, 2.0 M in hexane, 2.0 equiv), and 4-(trifluoromethyl)benzaldehyde (1.306 g, $7.5 \mathrm{mmol}, 1.5$ equiv) in $20 \mathrm{~mL}$ of THF. The resulted mixture was further oxidized by IBX (3.500 g, 12.5 mmol, 2.5 equiv) in $20 \mathrm{~mL}$ of DMSO to afford $\mathbf{1 h}$ (1.732 g, 79\%) as a yellow solid. (Known compound)

\section{2,4,4-triphenyl-1-(thiophen-2-yl)buta-2,3-dien-1-one (1i)}

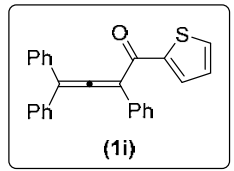

The reaction of prop-2-yne-1,1,3-triyltribenzene (1.342 g, 5.0 mmol, 1.0 equiv), LDA (5 mL, 2.0 M in hexane, 2.0 equiv), and 2-thiophenaldehyde (0.841 g, $7.5 \mathrm{mmol}, 1.5$ equiv) in $20 \mathrm{~mL}$ of THF. The resulted mixture was further oxidized by IBX (3.500 g, $12.5 \mathrm{mmol}, 2.5$ equiv) in $20 \mathrm{~mL}$ of DMSO to afford $1 \mathbf{i}$ (1.325 g, 70\%) as a yellow solid. (Known compound)

\section{2,4,4-triphenyl-1-(thiophen-2-yl)buta-2,3-dien-1-one (1j)}

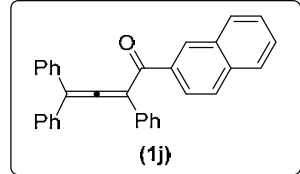

The reaction of prop-2-yne-1,1,3-triyltribenzene (1.342 g, 5.0 mmol, 1.0 equiv), LDA (5 mL, 2.0 M in hexane, 2.0 equiv), and 2-naphthaldehyde (1.172 g, 7.5 mmol, 1.5 equiv) in $20 \mathrm{~mL}$ of THF. The resulted mixture was further oxidized by IBX (3.500 g, 12.5 mmol, 2.5 equiv) in $20 \mathrm{~mL}$ of DMSO to afford $\mathbf{1} \mathbf{j}$ (1.622 g, 77\%) as a yellow solid. (Known compound)

\section{1-(4-methoxyphenyl)-2,4,4-triphenylbuta-2,3-dien-1-one (1k)}

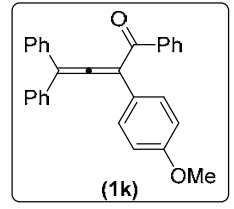

The reaction of (3-(4-methoxyphenyl)prop-2-yne-1,1-diyl)dibenzene (1.490g, 5.0 mmol, 1.0 equiv), LDA (5 mL, 2.0 M in hexane, 2.0 equiv), and benzaldehyde (0.795g, $7.5 \mathrm{mmol}, 1.5$ equiv) in $20 \mathrm{~mL}$ of THF. The resulted mixture was further oxidized by IBX (3.500 g, 12.5 mmol, 2.5 equiv) in $20 \mathrm{~mL}$ of DMSO to afford $\mathbf{1 k}$ (1.208 g, 60\%) as a yellow solid; m.p. 66-68 ${ }^{\circ} \mathrm{C}$ (Petroleum ether/EtOAc); $\mathrm{R} f=0.25$ (Petroleum ether/EtOAc $\left.=10 / 1\right) ;{ }^{1} \mathrm{H}$ NMR $(400$ 
$\left.\mathrm{MHz}, \mathrm{CDCl}_{3}\right): \delta$ 7.79-7.77 (m, 2H), 7.49-7.45 (m,3H), 7.35-7.32 (m, 6H), 7.24-7.21 (m, 6H), 6.93-6.90 (m, 2H), 3.82 (s, 3H); ${ }^{13} \mathrm{C}$ NMR (100 MHz, $\left.\mathrm{CDCl}_{3}\right): \delta 212.7,193.5,159.5,138.2,135.0$, 132.8, 129.31, 129.27, 128.7, 128.5, 128.14, 128.09, 125.0, 115.5, 114.2, 110.9, 55.3; HRMS (ES ${ }^{+}$-TOF) calcd for $\mathrm{C}_{29} \mathrm{H}_{23} \mathrm{O}_{2}\left([\mathrm{M}+\mathrm{H}]^{+}\right)$: 403.1693, found 403.1687 .

\section{2-(4-chlorophenyl)-1,4,4-triphenylbuta-2,3-dien-1-one (11)}

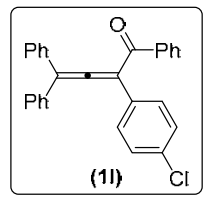

The reaction of (3-(4-chlorophenyl)prop-2-yne-1,1-diyl)dibenzene (1.514g, $5.0 \mathrm{mmol}$, 1.0 equiv), LDA (5 mL, 2.0 M in hexane, 2.0 equiv), and benzaldehyde (0.795g, 7.5 mmol, 1.5 equiv) in $20 \mathrm{~mL}$ of THF. The resulted mixture was further oxidized by IBX (3.500 g, 12.5 mmol, 2.5 equiv) in $20 \mathrm{~mL}$ of DMSO to afford $\mathbf{1 l}(1.607 \mathrm{~g}, 79 \%)$ as a yellow solid; m.p. $72-74{ }^{\circ} \mathrm{C}$ (Petroleum ether/EtOAc); R $f=0.45$ (Petroleum ether/EtOAc $=10 / 1) ;{ }^{1} \mathrm{H}$ NMR $\left(400 \mathrm{MHz}, \mathrm{CDCl}_{3}\right): \delta$ 7.77-7.75 (m, 2H), 7.50-7.44 (m, 3H), 7.37-7.34 (m, 8H), 7.24-7.21 (m, 6H); ${ }^{13} \mathrm{C}$ NMR (100 MHz, $\left.\mathrm{CDCl}_{3}\right): \delta 213.1,192.9,137.9,134.5,133.9,133.0,131.5,129.4,129.3,128.9,128.8,128.5,128.4$, 128.2, 116.0, 110.3; HRMS (ES ${ }^{+}$-TOF) calcd for $\mathrm{C}_{28} \mathrm{H}_{20} \mathrm{ClO}\left([\mathrm{M}+\mathrm{H}]^{+}\right)$: 407.1197, found 407.1202.

\section{2-(4-bromophenyl)-1,4,4-triphenylbuta-2,3-dien-1-one (1m)}

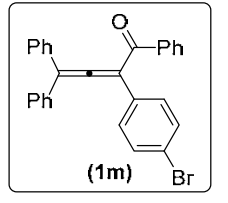

The reaction of (3-(4-bromophenyl)prop-2-yne-1,1-diyl)dibenzene (1.737 g, 5.0 mmol, 1.0 equiv), LDA (5 mL, 2.0 $\mathrm{M}$ in hexane, 2.0 equiv), and benzaldehyde (0.796 g, 7.5 mmol, 1.5 equiv) in $20 \mathrm{~mL}$ of THF. The resulted mixture was further oxidized by IBX (3.500 g, 12.5 mmol, 2.5 equiv) in $20 \mathrm{~mL}$ of DMSO to afford $\mathbf{1 m}(1.625 \mathrm{~g}, 72 \%)$ as a yellow solid. (Known compound)

\section{2-(3-fluorophenyl)-1,4,4-triphenylbuta-2,3-dien-1-one (1n)}

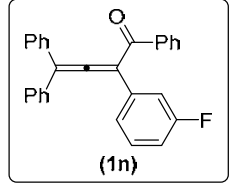

The reaction of (3-(3-fluorophenyl)prop-2-yne-1,1-diyl)dibenzene (1.431 g, 5.0 mmol, 1.0 equiv), LDA (5 mL, 2.0 $\mathrm{M}$ in hexane, 2.0 equiv), and benzaldehyde (0.796 
g, 7.5 mmol, 1.5 equiv) in $20 \mathrm{~mL}$ of THF. The resulted mixture was further oxidized by IBX (3.500 g, $12.5 \mathrm{mmol}, 2.5$ equiv) in $20 \mathrm{~mL}$ of DMSO to afford $\mathbf{1 n}(0.918 \mathrm{~g}, 47 \%)$ as a yellow solid. (Known compound)

\section{2-(4-bromophenyl)-4,4-diphenyl-1-(p-tolyl)buta-2,3-dien-1-one (1p)}

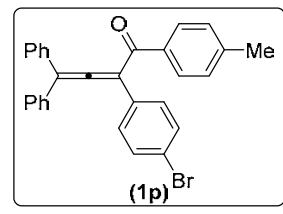

The reaction of (3-(4-bromophenyl)prop-2-yne-1,1-diyl)dibenzene (1.736g, 5.0 mmol, 1.0 equiv), LDA (5 mL, 2.0 $\mathrm{M}$ in hexane, 2.0 equiv), and 4-methylbenzaldehyde (0.901g, $7.5 \mathrm{mmol}, 1.5$ equiv) in $20 \mathrm{~mL}$ of THF. The resulted mixture was further oxidized by IBX (3.500g, $12.5 \mathrm{mmol}, 2.5$ equiv) in $20 \mathrm{~mL}$ of DMSO to afford 1p (1.652 g, 71\%) as a yellow solid; m.p. $107-109{ }^{\circ} \mathrm{C}$ (Petroleum ether/EtOAc); $\mathrm{R} f=0.45$ (Petroleum ether/EtOAc $=10 / 1) ;{ }^{1} \mathrm{H}$ NMR $\left(400 \mathrm{MHz}, \mathrm{CDCl}_{3}\right): \delta$ 7.71-7.69 (m, 2H), 7.50-7.47 (m, 2H), 7.41-7.34 (m, 7H), 7.27-7.24 (m, 5H), 7.03 (d, $J=8.0 \mathrm{~Hz}, 2 \mathrm{H}), 2.33$ (s, 3H); ${ }^{13} \mathrm{C}$ NMR $\left(100 \mathrm{MHz}, \mathrm{CDCl}_{3}\right): \delta$ 212.2, 192.3, 144.1, 135.1, 134.6, 132.2, 131.8, 129.6, 128.9, 128.7, 128.5, 128.4, 122.0, 115.9, 110.2, 21.6; HRMS (ES $\left.{ }^{+}-\mathrm{TOF}\right)$ calcd for $\mathrm{C}_{29} \mathrm{H}_{22} \mathrm{BrO}\left([\mathrm{M}+\mathrm{H}]^{+}\right)$: 465.0849, found 465.0830 .

\section{4,4-bis(4-methoxyphenyl)-1,2-diphenylbuta-2,3-dien-1-one (1q)}

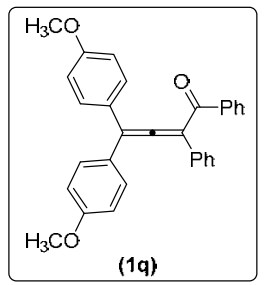

The reaction of 4,4'-(3-phenylprop-2-yne-1,1-diyl)bis(methoxybenzene) (1.642 g, $5.0 \mathrm{mmol}, 1.0$ equiv), LDA (5 mL, 2.0 M in hexane, 2.0 equiv), and benzaldehyde (0.796 g, $7.5 \mathrm{mmol}, 1.5$ equiv) in $20 \mathrm{~mL}$ of THF. The resulted mixture was further oxidized by IBX (3.500 g, 12.5 mmol, 2.5 equiv) in 20 mL of DMSO to afford 1q (0.908 g, 42\%) a yellow solid. (Known compound)

\section{4,4-bis(4-chlorophenyl)-1,2-diphenylbuta-2,3-dien-1-one (1r)}

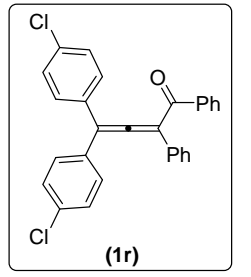

The reaction of 4,4'-(3-phenylprop-2-yne-1,1-diyl)bis(chlorobenzene) (1.886 g, 5.0 mmol, 1.0 equiv), LDA (5 mL, 2.0 $\mathrm{M}$ in hexane, 2.0 equiv), and benzaldehyde (0.796 
g, 7.5 mmol, 1.5 equiv) in $20 \mathrm{~mL}$ of THF. The resulted mixture was further oxidized by IBX (3.500 g, $12.5 \mathrm{mmol}, 2.5$ equiv) in $20 \mathrm{~mL}$ of DMSO to afford $1 \mathbf{r}(1.368 \mathrm{~g}, 62 \%)$ as a yellow solid. (Known compound)

\section{3-(10,11-dihydro-5H-dibenzo[a,d][7]annulen-5-ylidene)-1,2-diphenylprop-2-en-1-one (1s)}

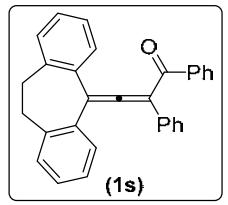

The reaction of 5-(phenylethynyl)-10,11-dihydro-5H-dibenzo[a,d][7]annulene

(1.473g, $5.0 \mathrm{mmol}, 1.0$ equiv), LDA (5 mL, $2.0 \mathrm{M}$ in hexane, 2.0 equiv), and benzaldehyde (0.795g, $7.5 \mathrm{mmol}, 1.5$ equiv) in $20 \mathrm{~mL}$ of THF. The resulted mixture was further oxidized by IBX (3.500 g, 12.5 mmol, 2.5 equiv) in $20 \mathrm{~mL}$ of DMSO to afford $1 \mathrm{~s}$ (1.415 g, $71 \%$ ) as a yellow solid; m.p. 86-89 ${ }^{\circ} \mathrm{C}$ (Petroleum ether/EtOAc); $\mathrm{R} f=0.45$ (Petroleum ether/EtOAc $=$ 10/1); ${ }^{1} \mathrm{H}$ NMR (400 MHz, $\mathrm{CDCl}_{3}$ ): $\delta 7.74$ (d, $\left.J=7.6 \mathrm{~Hz}, 2 \mathrm{H}\right), 7.65$ (d, $\left.J=7.6 \mathrm{~Hz}, 2 \mathrm{H}\right)$, 7.47-7.34 (m, 6H), 7.23-7.10 (m, 8H), 2.85-2.73 (m, 4H); ${ }^{13} \mathrm{C}$ NMR (100 MHz, $\left.\mathrm{CDCl}_{3}\right): \delta 213.2,193.4,139.1$, 138.5, 134.7, 133.1, 132.6, 129.6, 129.4, 129.2, 128.8, 128.4, 128.0, 127.8, 126.5, 117.1, 109.1, 33.1; HRMS (ES ${ }^{+}$TOF) calcd for $\mathrm{C}_{30} \mathrm{H}_{23} \mathrm{O}\left([\mathrm{M}+\mathrm{H}]^{+}\right)$: 399.1743, found.399.1747.

\section{1-(4-bromophenyl)-2-phenyl-4,4-di-p-tolylbuta-2,3-dien-1-one (1t)}

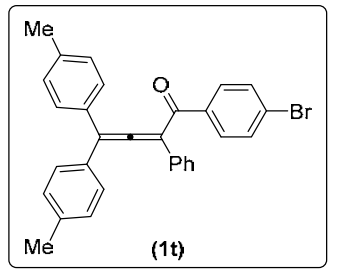

The reaction of 4,4'-(3-(4-bromophenyl)prop-2-yne-1,1-diyl)bis(methylbenzene)

(1.871 g, $5.0 \mathrm{mmol}, 1.0$ equiv), LDA (5 mL, 2.0 $\mathrm{M}$ in hexane, 2.0 equiv), and 4-bromobenzaldehyde (1.388 g, $7.5 \mathrm{mmol}, 1.5$ equiv) in $20 \mathrm{~mL}$ of THF. The resulted mixture was further oxidized by IBX (3.500 g, $12.5 \mathrm{mmol}, 2.5$ equiv) in $20 \mathrm{~mL}$ of DMSO to afford 1t (1.758 g, 71\%) as a yellow solid; m.p. $113-115{ }^{\circ} \mathrm{C}$ (Petroleum ether/EtOAc); $\mathrm{R} f=0.45$ $($ Petroleum ether/EtOAc $=10 / 1) ;{ }^{1} \mathrm{H}$ NMR $\left(400 \mathrm{MHz}, \mathrm{CDCl}_{3}\right): \delta 7.64(\mathrm{~d}, J=8.4 \mathrm{~Hz}, 2 \mathrm{H}), 7.52(\mathrm{~d}, J=$ 8.0 Hz, 2H), 7.38-7.35 (m, 4H), 7.32-7.30 (m, 1H), 7.17-7.12 (m, 8H), 2.37 (m, 6H); ${ }^{13} \mathrm{C}$ NMR (100 $\left.\mathrm{MHz}, \mathrm{CDCl}_{3}\right): \delta$ 213.2, 192.2, 138.3, 136.9, 133.0, 131.7, 131.4, 130.8, 129.5, 128.7, 128.4, 128.1, 
128.0, 127.9, 115.7, 110.8, 21.2; HRMS (ES ${ }^{+}$-TOF) calcd for $\mathrm{C}_{30} \mathrm{H}_{24} \mathrm{BrO}\left([\mathrm{M}+\mathrm{H}]^{+}\right)$: 479.1005, found 479.1015.

\section{2,4,4-tris(4-chlorophenyl)-1-phenylbuta-2,3-dien-1-one (1u)}

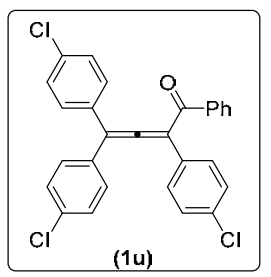

The reaction of 4,4',4"-(prop-2-yne-1,1,3-triyl)tris(chlorobenzene) (1.859 g, 5.0 mmol, 1.0 equiv), LDA (5 mL, 2.0 M in hexane, 2.0 equiv), and benzaldehyde (0.796 g, $7.5 \mathrm{mmol}, 1.5$ equiv) in $20 \mathrm{~mL}$ of THF. The resulted mixture was further oxidized by IBX (3.502 g, $12.5 \mathrm{mmol}, 2.5$ equiv) in $20 \mathrm{~mL}$ of DMSO to afford 1u (1.665 g, 70\%) as a yellow solid; m.p. $121-122{ }^{\circ} \mathrm{C}$ (Petroleum ether/EtOAc); $\mathrm{R} f=0.45$ (Petroleum ether/EtOAc $=$ 10/1); ${ }^{1} \mathrm{H}$ NMR (400 MHz, $\left.\mathrm{CDCl}_{3}\right): \delta 7.75$ (d, $\left.J=7.2 \mathrm{~Hz}, 2 \mathrm{H}\right), 7.50$ (t, J = 7.2 Hz, 1H), 7.42 (d, $J=$ 8.4 Hz, 2H), 7.36-7.33 (m, 6H), 7.29-7.27 (m, 2H), 7.14 (d, $J=8.4 \mathrm{~Hz}, 4 \mathrm{H}) ;{ }^{13} \mathrm{C}$ NMR (100 MHz, $\left.\mathrm{CDCl}_{3}\right): \delta 212.0,192.4,137.7,134.6,134.3,133.3,132.8,130.9,129.6,129.24,129.15,129.1,128.3$, 114.3, 110.9; HRMS (ES ${ }^{+}$TOF) calcd for $\mathrm{C}_{28} \mathrm{H}_{18} \mathrm{Cl}_{3} \mathrm{O}\left([\mathrm{M}+\mathrm{H}]^{+}\right)$: 475.0418, found 475.0418.

\section{1,2,4-triphenyl-4-(p-tolyl)buta-2,3-dien-1-one (1v)}

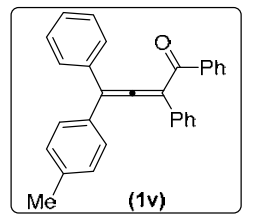

The reaction of (3-(p-tolyl)prop-1-yne-1,3-diyl)dibenzene (1.412 g, $5.0 \mathrm{mmol}, 1.0$ equiv), LDA (5 mL, 2.0 M in hexane, 2.0 equiv), and benzaldehyde (0.796 g, 7.5 mmol, 1.5 equiv) in $20 \mathrm{~mL}$ of THF. The resulted mixture was further oxidized by IBX (3.500 g, 12.5 mmol, 2.5 equiv) in $20 \mathrm{~mL}$ of DMSO to afford $\mathbf{1 v}(1.353 \mathrm{~g}, 70 \%)$ as a yellow solid. (Known compound)

\section{4-(4-bromophenyl)-1,2,4-triphenyl-3l5-buta-2,3-dien-1-one (1w)}

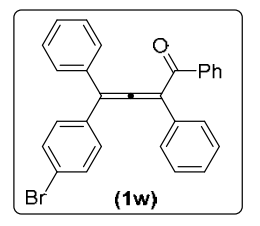

The reaction of (3-(4-bromophenyl)prop-1-yne-1,3-diyl)dibenzene (1.736 g, 5.0 mmol, 1.0 equiv), LDA (5 mL, 2.0 $\mathrm{M}$ in hexane, 2.0 equiv), and benzaldehyde (0.796 g, $7.5 \mathrm{mmol}, 1.5$ equiv) in $20 \mathrm{~mL}$ of THF. The resulted mixture was further oxidized by IBX 
(3.502 g, $12.5 \mathrm{mmol}, 2.5$ equiv) in $20 \mathrm{~mL}$ of DMSO to afford $\mathbf{1 w}(1.422 \mathrm{~g}, 63 \%$ ) as a yellow solid; m.p. $136-137{ }^{\circ} \mathrm{C}$ (Petroleum ether/EtOAc); $\mathrm{R} f=0.40$ (Petroleum ether/EtOAc $\left.=10 / 1\right) ;{ }^{1} \mathrm{H}$ NMR $(400$ $\left.\mathrm{MHz}, \mathrm{CDCl}_{3}\right): \delta$ 7.80-7.78 (m, 2H), 7.52-7.46 (m, 5H), 7.38-7.31 (m, 6H) 7.25-7.24 (m, 4H), 7.15-7.12 (m, 2H); ${ }^{13} \mathrm{C}$ NMR (100 MHz, $\left.\mathrm{CDCl}_{3}\right): \delta 212.0,192.9,137.9,134.4,133.9,133.1,132.7$, 131.9, 130.0, 129.3, 128.8, 128.7, 128.4, 128.2, 127.9, 122.3, 114.9, 111.5, 106.0; HRMS (ES ${ }^{+}$-TOF) calcd for $\mathrm{C}_{28} \mathrm{H}_{20} \mathrm{BrO}\left([\mathrm{M}+\mathrm{H}]^{+}\right)$: 451.0692, found 451.0692 .

\section{4-(4-chlorophenyl)-1,2-diphenyl-4-(p-tolyl)buta-2,3-dien-1-one (1x)}

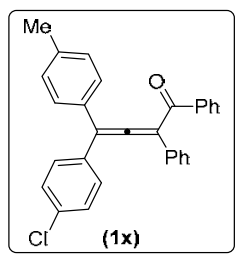

The reaction of 1-chloro-4-(3-phenyl-1-(p-tolyl)prop-2-yn-1-yl)benzene (1.584g, 5.0 mmol, 1.0 equiv), LDA (5 mL, 2.0 $\mathrm{M}$ in hexane, 2.0 equiv), and benzaldehyde (0.795g, $7.5 \mathrm{mmol}, 1.5$ equiv) in $20 \mathrm{~mL}$ of THF. The resulted mixture was further oxidized by IBX (3.500 g, $12.5 \mathrm{mmol}, 2.5$ equiv) in $20 \mathrm{~mL}$ of DMSO to afford $\mathbf{1 x}$ (1.368 g, 65\%) as a yellow solid; m.p. $118-121^{\circ} \mathrm{C}$ (Petroleum ether/EtOAc); $\mathrm{R} f=0.45$ (Petroleum ether/EtOAc $=$ 10/1); ${ }^{1} \mathrm{H}$ NMR (400 MHz, $\left.\mathrm{CDCl}_{3}\right): \delta$ 7.80-7.78 (m, 2H), 7.52-7.45 (m, 3H), 7.39-7.35 (m, 2H), 7.32-7.25 (m, 5H), 7.20-7.11 (m, 6H), 2.37 (s, 3H); ${ }^{13} \mathrm{C}$ NMR (100 MHz, $\left.\mathrm{CDCl}_{3}\right): \delta$ 212.1, 193.0, 138.4, 138.0, 134.1, 133.6, 133.0, 132.9, 131.4, 129.7, 129.5, 129.3, 128.9, 128.8, 128.4, 128.3, 128.2, 128.1, 128.0, 114.7, 111.4, 21.2; HRMS (ES ${ }^{+}$TOF) calcd for $\mathrm{C}_{29} \mathrm{H}_{22} \mathrm{ClO}\left([\mathrm{M}+\mathrm{H}]^{+}\right)$: 421.1354, found 421.1365 .

\section{4-(4-chlorophenyl)-4-(4-methoxyphenyl)-1,2-diphenylbuta-2,3-dien-1-one (1y)}

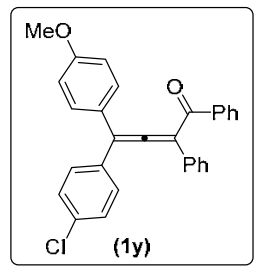

The reaction of 1-chloro-4-(1-(4-methoxyphenyl)-3-phenylprop-2-yn-1-yl)benzene

(1.664 g, $5.0 \mathrm{mmol}, 1.0$ equiv), LDA (5 mL, 2.0 M in hexane, 2.0 equiv), and benzaldehyde (0.795g, $7.5 \mathrm{mmol}, 1.5$ equiv) in $20 \mathrm{~mL}$ of THF. The resulted mixture was further oxidized by IBX (3.500 g, 12.5 mmol, 2.5 equiv) in 20 mL of DMSO to afford 1y (1.529 
g, 70\%) as a yellow solid; m.p. 89-91 ${ }^{\circ} \mathrm{C}$ (Petroleum ether/EtOAc); $\mathrm{R} f=0.45$ (Petroleum ether/EtOAc = 10/1); ${ }^{1} \mathrm{H}$ NMR $\left(400 \mathrm{MHz}, \mathrm{CDCl}_{3}\right): \delta$ 7.80-7.78 (m, 2H), 7.52-7.45 (m, 3H), 7.40-7.36 (m, 2H), 7.32-7.24 (m, 5H), 7.20-7.19 (m, 1H), 7.18-7.15 (m, 3H), 6.90-6.87 (m, 2H), 3.82 (s, 3H);

${ }^{13} \mathrm{C}$ NMR (100 MHz, $\left.\mathrm{CDCl}_{3}\right): \delta$ 212.1, 193.1, 159.8, 138.0, 134.1, 133.7, 133.0, 132.9, 129.7, 129.6, 129.3, 128.9, 128.8, 128.2, 128.1, 127.9, 126.5, 114.5, 114.3, 111.3, 55.3; HRMS (ES $\left.{ }^{+}-\mathrm{TOF}\right)$ calcd for $\mathrm{C}_{29} \mathrm{H}_{22} \mathrm{ClO}_{2}\left([\mathrm{M}+\mathrm{H}]^{+}\right)$: 437.1303, found 437.1305.

\subsection{Synthesis of Starting Materials 1z.}

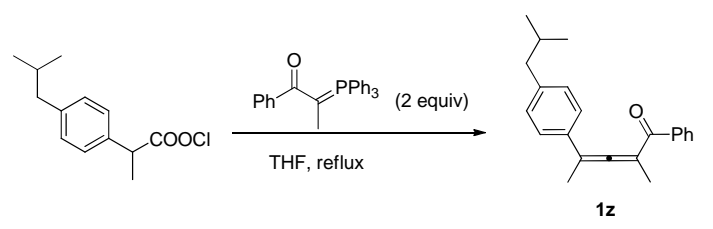

4-(4-isobutylphenyl)-2-methyl-1-phenylpenta-2,3-dien-1-one (1z)

To a solution of 1-phenyl-2-(triphenyl-15-phosphanylidene)propan-1-one (3.375 g, $8.6 \mathrm{mmol}, 2.0$ equiv) in dry THF (30 mL) under $\mathrm{N}_{2}$ was added to hypochlorous 2-(4-isobutylphenyl)propanoic anhydride (0.963 g, $4.3 \mathrm{mmol}, 1.0$ equiv), then the solution was refluxed for $4.0 \mathrm{~h}$. After completion of the reaction, the mixture was quenched by adding $50 \mathrm{~mL}$ of $\mathrm{H}_{2} \mathrm{O}$ and extracted with EtOAc $(3 \times 50$ $\mathrm{mL})$. The combined organic phase was washed with $\mathrm{H}_{2} \mathrm{O}(3 \times 30 \mathrm{~mL})$, dried over anhydrous $\mathrm{Na}_{2} \mathrm{SO}_{4}$, concentrated in vacuo and purified by flash silica gel chromatography to afford 1z. (795 mg, 60\%) as a red liquid; $\mathrm{R} f=0.40$ (Petroleum ether/EtOAc $=10 / 1) ;{ }^{1} \mathrm{H}$ NMR $\left(400 \mathrm{MHz}, \mathrm{CDCl}_{3}\right): \delta$ 7.72-7.70 (m, 2H), 7.42-7.39 (m, 1H), 7.28 (d, $J=7.6 \mathrm{~Hz}, 2 \mathrm{H}), 7.17$ (d, $J=8.4 \mathrm{~Hz}, 2 \mathrm{H}), 7.09$ (d, $J=8.0 \mathrm{~Hz}, 2 \mathrm{H})$, 2.45 (d, $J=7.2 \mathrm{~Hz}, 2 \mathrm{H}), 2.11(\mathrm{~d}, J=8.0 \mathrm{~Hz}, 6 \mathrm{H}), 1.88-1.81(\mathrm{~m}, 1 \mathrm{H}), 0.90(\mathrm{~s}, 3 \mathrm{H}), 0.88(\mathrm{~s}, 3 \mathrm{H}) ;{ }^{13} \mathrm{C}$ NMR (100 MHz, $\left.\mathrm{CDCl}_{3}\right): \delta$ 214.6, 195.2, 141.2, 138.6, 132.6, 131.7, 129.3, 128.5, 127.8, 125.7, 104.0, 103.1, 45.0, 30.2, 22.3, 16.5, 15.0; HRMS (ES ${ }^{+}$TOF) calcd for $\mathrm{C}_{22} \mathrm{H}_{25} \mathrm{O}\left([\mathrm{M}+\mathrm{H}]^{+}\right)$: 305.1900, found 305.1911. 


\section{X-ray diffraction analysis of $2 p, 2 y, 5 a, 9$, and 10}

Crystallographic structure analysis of 2p, 2y, 5a, 9 and 10: A suitable single crystal was mounted on a Xcalibur, Atlas, Gemini ultra at $\mathrm{T}_{(\mathbf{2 p})}=150 \mathrm{~K}, \mathrm{~T}_{(\mathbf{2 y})}=293 \mathrm{~K}, \mathrm{~T}_{(\mathbf{5 a})}=298 \mathrm{~K}, \mathrm{~T}_{(\mathbf{9 )}}=170 \mathrm{~K}$ or $\mathrm{T}_{(\mathbf{1 0 )}}=$ $298 \mathrm{~K}$ using Mo Ka radiation $\left(\lambda_{(\mathbf{2 p})}=0.71073 \AA, \lambda_{(\mathbf{2 y})}=0.71073 \AA, \lambda_{(\mathbf{5 a})}=0.71073 \AA, \lambda_{(\mathbf{9})}=0.71073\right.$ $\left.\AA, \lambda_{(\mathbf{1 0 )}}=0.71073 \AA\right)$. The intensity data were collected with CrysAlisPro program and reduced by CrysAlisPro program. The structure was solved by direct methods, expended by difference Fourier syntheses and refined by Full-matrix squares on $\mathrm{F}^{2}$ using SHELXL program packages. All non-hydrogen atoms were refined anisotropically. Hydrogen atoms were placed in ideal positions and refined as riding atoms. Details of the X-ray experiments and crystal data are summarized in

Table S2, Table S3, Table S4, Table S5 and Table S6.

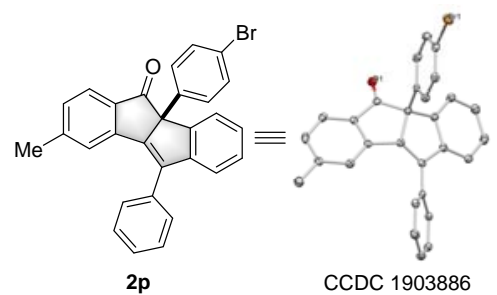

Figure S7. ORTEP drawing of 2p with ellipsoid contour at 30\% probability level

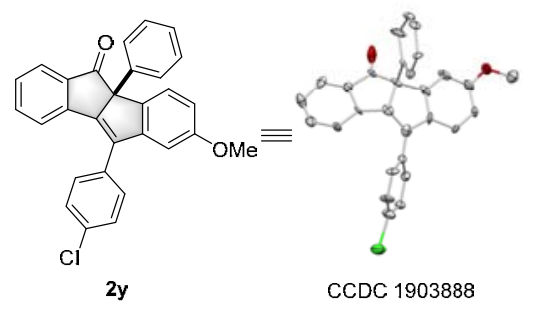

Figure S8. ORTEP drawing of $\mathbf{2 y}$ with ellipsoid contour at 30\% probability level

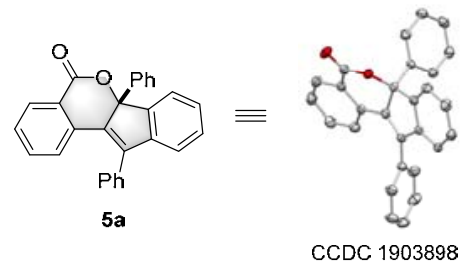

Figure S9. ORTEP drawing of 5a with ellipsoid contour at 30\% probability level 


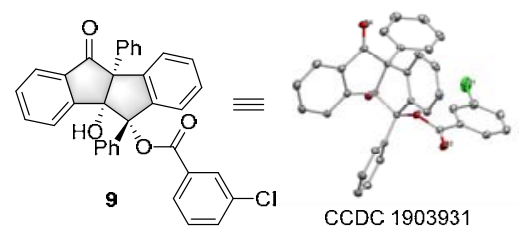

Figure S10. ORTEP drawing of 9 with ellipsoid contour at 30\% probability level

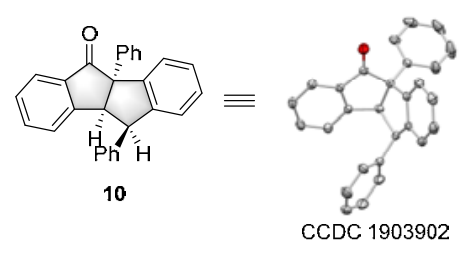

Figure S11. ORTEP drawing of $\mathbf{1 0}$ with ellipsoid contour at 30\% probability level

Table S2. Crystal data and structure refinement for $\mathbf{2 p}$.

Empirical formula

Formula weight

Temperature

Wavelength

Crystal system, Space group

Unit cell dimensions

Volume

Z

Density(calculated)

Absorption coefficient

$\mathrm{F}(000)$

Crystal size

Theta range for data collection

Index ranges

Reflections collected

Independent reflections

Completeness to theta $=25.350^{\circ}$
$\mathrm{C}_{29} \mathrm{H}_{19} \mathrm{BrO}$

463.35

$150 \mathrm{~K}$

$0.71073 \AA$

trigonal, R -3

$\mathrm{a}=28.0155(18) \AA \quad \alpha=90^{\circ}$

$\mathrm{b}=28.0155(18) \AA \quad \beta=90^{\circ}$

$\mathrm{c}=14.9771(11) \AA \quad \gamma=120^{\circ}$

10180.1(15) $\AA^{3}$

18

$1.360 \mathrm{Mg} / \mathrm{m}^{3}$

$1.835 \mathrm{~mm}^{-1}$

4248

$0.42 * 0.36 * 0.29 \mathrm{~mm}^{3}$

3.197 to $25.349^{\circ}$.

$-18<=\mathrm{h}<=33,-33<=\mathrm{k}<=28,-17<=\mathrm{l}<=14$

$6978[\mathrm{R}(\mathrm{int})=0.0382]$

4126

$99.6 \%$ 

Absorption correction
Multi-scan from equivalents
Refinement method
Full-matrix squares on $\mathrm{F}^{2}$
Data/restraints/parameters
$4126 / 0 / 281$
Goodness-of-fit on $\mathrm{F}^{2}$
1.026
Final R indices [I $>2 \operatorname{sigma}(\mathrm{I})]$
$\mathrm{R}_{1}=0.0438, \mathrm{wR}_{2}=0.0924$
$\mathrm{R}$ indices(all data)
$\mathrm{R}_{1}=0.0656, \mathrm{wR}_{2}=0.1045$
Extinction coefficient
?
Largest diff. peak and hole
0.606 and $-0.547 \AA^{3}$

Table S3. Crystal data and structure refinement for $\mathbf{2 y}$.

Empirical formula

Formula weight

Temperature

Wavelength

Crystal system, Space group

Unit cell dimensions

Volume

Z

Density(calculated)

Absorption coefficient

$\mathrm{F}(000)$

Crystal size

Theta range for data collection

Index ranges

Reflections collected

Independent reflections
$\mathrm{C}_{29} \mathrm{H}_{19} \mathrm{ClO}_{2}$

434.11

293K

$0.71073 \AA$

monoclinic, P 1 21/m 1

$$
\begin{array}{ll}
a=11.7014(12) \AA & \alpha=90^{\circ} \\
b=8.6987(8) \AA & \beta=94.856(9)^{\circ} \\
c=21.7780(2) \AA & \gamma=90^{\circ}
\end{array}
$$

2208.7(4) $\AA^{3}$

2

$1.987 \mathrm{Mg} / \mathrm{m}^{3}$

$1.341 \mathrm{~mm}^{-1}$

1312.0

?

5.844 to $45^{\circ}$

$-11<=\mathrm{h}<=12,-9<=\mathrm{k}<=7,-23<=\mathrm{l}<=21$

$6550[\mathrm{R}(\mathrm{int})=0.0547]$

3954 
Completeness to theta $=25.350^{\circ}$

Absorption correction

Refinement method

Data/restraints/parameters

Goodness-of-fit on $\mathrm{F}^{2}$

Final R indices [I>2sigma(I)]

$\mathrm{R}$ indices(all data)

Extinction coefficient

Largest diff. peak and hole
$99.77 \%$

Multi-scan from equivalents

Full-matrix squares on $\mathrm{F}^{2}$

$3954 / 2 / 579$

2.246

$\mathrm{R}_{1}=0.1747, \mathrm{wR}_{2}=0.4869$

$\mathrm{R}_{1}=0.1879, \mathrm{wR}_{2}=0.5017$

?

0.83 and $-0.60 \AA^{3}$

Table S4. Crystal data and structure refinement for $\mathbf{5 a}$.

Empirical formula

Formula weight

Temperature

Wavelength

Crystal system, Space group

Unit cell dimensions

Volume

Z

Density(calculated)

Absorption coefficient

$\mathrm{F}(000)$

Crystal size

Theta range for data collection

Index ranges

Reflections collected
$\mathrm{C}_{28} \mathrm{H}_{18} \mathrm{O}_{2}$

386.45

$298 \mathrm{~K}$

$0.71073 \AA$

monoclinic, P 1 21/n 1

$$
\begin{array}{ll}
\mathrm{a}=11.7597(9) \AA & \alpha=90^{\circ} \\
\mathrm{b}=10.1515(7) \AA & \beta=105.118(7)^{\circ} \\
\mathrm{c}=16.9119(11) \AA & \gamma=90^{\circ}
\end{array}
$$

1949.0(3) $\AA^{3}$

4

$1.3169 \mathrm{Mg} / \mathrm{m}^{3}$

$0.082 \mathrm{~mm}^{-1}$

808.3831

?

3.16 to $29.38^{\circ}$

$-15<=\mathrm{h}<=15,-14<=\mathrm{k}<=7,-21<=\mathrm{l}<=14$

8659 [R(int $)=0.0291]$ 
Independent reflections

Completeness to theta $=25.350^{\circ}$

Absorption correction

Refinement method

Data/restraints/parameters

Goodness-of-fit on $\mathrm{F}^{2}$

Final R indices [I $>2 \operatorname{sigma(I)]~}$

$\mathrm{R}$ indices(all data)

Extinction coefficient

Largest diff. peak and hole
$99.76 \%$

Multi-scan from equivalents

Full-matrix squares on $\mathrm{F}^{2}$

$4522 / 0 / 270$

0.9907

$\mathrm{R}_{1}=0.0546, \mathrm{wR}_{2}=0.4869$

$\mathrm{R}_{1}=0.1155, \mathrm{wR}_{2}=0.1301$

?

0.3932 and $-0.4098 \AA^{3}$

Table S5. Crystal data and structure refinement for 9.

Empirical formula

Formula weight

Temperature

Wavelength

Crystal system, Space group

Unit cell dimensions

Volume

Z

Density(calculated)

Absorption coefficient

$\mathrm{F}(000)$

Crystal size

Theta range for data collection

Index ranges

Reflections collected
$\mathrm{C}_{35} \mathrm{H}_{23} \mathrm{ClO}_{4}$

542.13

$170 \mathrm{~K}$

$0.71073 \AA$

monoclinic, -P 2ybc

$$
\begin{array}{ll}
\mathrm{a}=8.9363(2) \AA & \alpha=90^{\circ} \\
\mathrm{b}=17.2110(4) \AA & \beta=101.2240(10)^{\circ} \\
\mathrm{c}=20.3755(4) \AA & \gamma=90^{\circ}
\end{array}
$$

3073.87(12) $\AA^{3}$

4

$1.364 \mathrm{Mg} / \mathrm{m}^{3}$

$0.174 \mathrm{~mm}^{-1}$

1320

?

2.577 to $25.350^{\circ}$

$-10<=\mathrm{h}<=10,-20<=\mathrm{k}<=20,-22<=\mathrm{l}<=24$

28853 [R(int) $=0.0368]$ 
Independent reflections

Completeness to theta $=25.350^{\circ}$

Absorption correction

Refinement method

Data/restraints/parameters

Goodness-of-fit on $\mathrm{F}^{2}$

Final R indices [I $>2 \operatorname{sigma}(\mathrm{I})]$

$\mathrm{R}$ indices(all data)

Extinction coefficient

Largest diff. peak and hole
5636

$99.6 \%$

Multi-scan from equivalents

Full-matrix squares on $\mathrm{F}^{2}$

$5636 / 0 / 418$

1.015

$\mathrm{R}_{1}=0.0546, \mathrm{wR}_{2}=0.0925$

$\mathrm{R}_{1}=0.0451, \mathrm{wR}_{2}=0.0994$

?

0.246 and $-0.388 \AA^{3}$

Table S6. Crystal data and structure refinement for $\mathbf{1 0 .}$

Empirical formula

Formula weight

Temperature

Wavelength

Crystal system, Space group

Unit cell dimensions

Volume

$\mathrm{Z}$

Density(calculated)

Absorption coefficient

$\mathrm{F}(000)$

Crystal size

Theta range for data collection

Index ranges

Reflections collected
$\mathrm{C}_{28} \mathrm{H}_{20} \mathrm{O}$

372.47

$298 \mathrm{~K}$

$0.71073 \AA$

triclinic, -P 1

$\mathrm{a}=10.1558(17) \AA \quad \alpha=83.002(10)^{\circ}$

$b=10.2913(8) \AA \quad \beta=62.589(17)^{\circ}$

$\mathrm{c}=10.8220(18) \AA \quad \gamma=85.427(9)^{\circ}$

996.3(3) $\AA^{3}$

2

$1.2416 \mathrm{Mg} / \mathrm{m}^{3}$

$0.074 \mathrm{~mm}^{-1}$

392.1694

?

2.77 to $29.47^{\circ}$

$-13<=\mathrm{h}<=13,-14<=\mathrm{k}<=9,-13<=\mathrm{l}<=14$

$8182[\mathrm{R}(\mathrm{int})=0.0279]$ 
Independent reflections

Completeness to theta $=25.350^{\circ}$

Absorption correction

Refinement method

Data/restraints/parameters

Goodness-of-fit on $\mathrm{F}^{2}$

Final R indices [I>2sigma(I)]

$\mathrm{R}$ indices(all data)

Extinction coefficient

Largest diff. peak and hole
4621

$99.80 \%$

Multi-scan from equivalents

Full-matrix squares on $\mathrm{F}^{2}$

$4621 / 0 / 261$

0.9598

$\mathrm{R}_{1}=0.0534, \mathrm{wR}_{2}=0.0925$

$\mathrm{R}_{1}=0.1029, \mathrm{wR}_{2}=0.1207$

?

0.3016 and $-0.3609 \AA^{3}$

\section{Reference}

1. M. Yasuda, T. Saito, M. Ueba, A. Baba, Angew. Chem. Int. Ed. 2004, 116, 1438-1440.

2. T. Johannes, P. Bernd, Org. Lett. 2018, 20, 2257-2260.

3. J. Shen, D. Yuan, Y. Qiao, X. Shen, Z. Zhang, Y. Zhong, Y. Yi, X. Zhu, Org. Lett. 2014, 16, 4924-4927.

4. H. Ren, M. Miao, H. Xu, Y. Luo, M. Jin, Z. Chen, J. Xu, Synthesis 2017, 50, 349-360. 
$\mathrm{HNMR}\left(400 \mathrm{MHz}, \mathrm{CDCl}_{3}\right)$
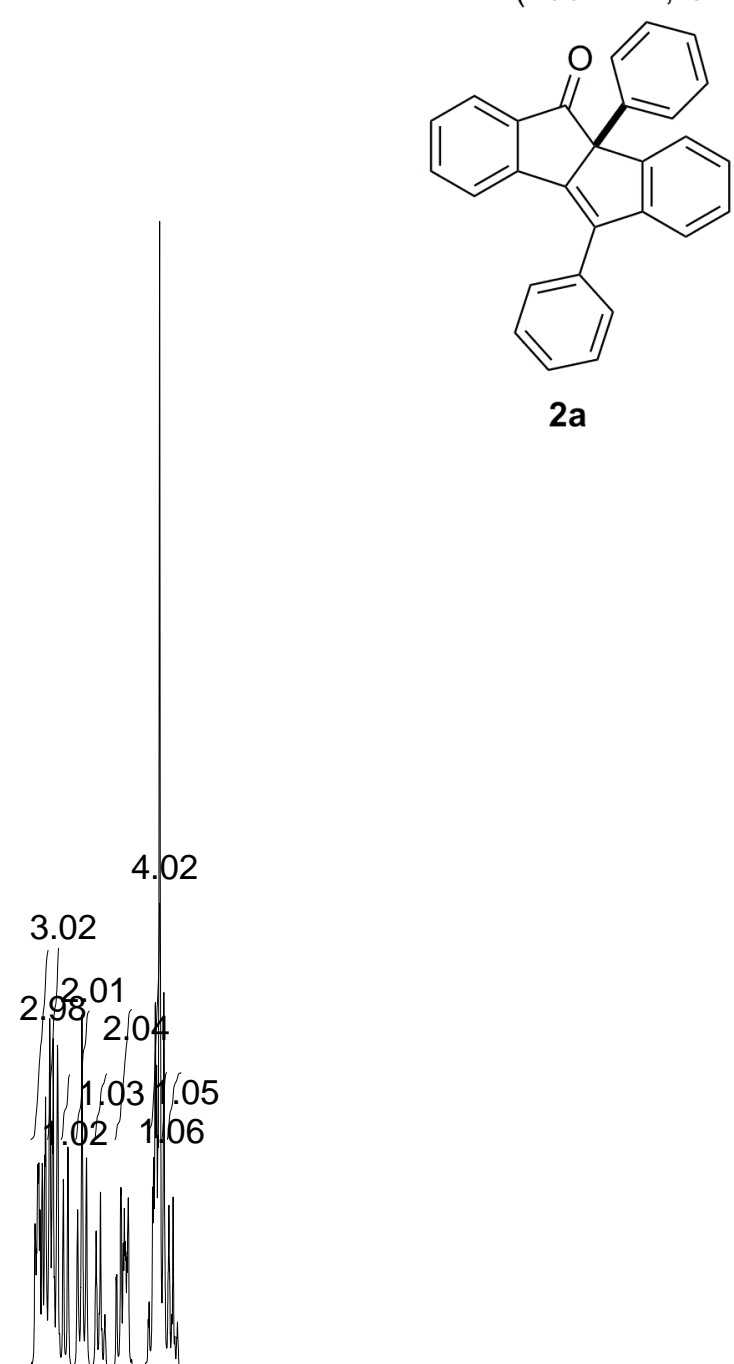

2a 
${ }^{13} \mathrm{C} \mathrm{NMR}\left(100 \mathrm{MHz}, \mathrm{CDCl}_{3}\right)$

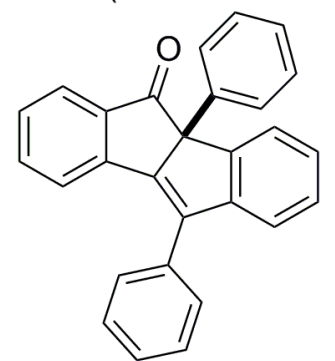

2a 


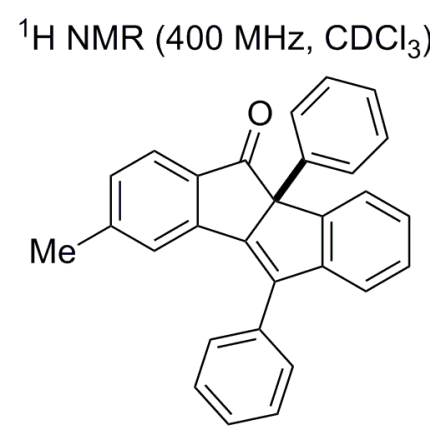

2b 
${ }^{13} \mathrm{C}$ NMR $\left(100 \mathrm{MHz}, \mathrm{CDCl}_{3}\right)$

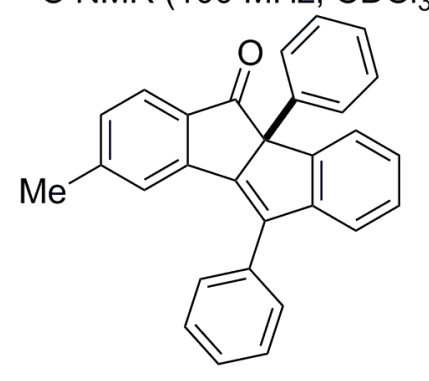

$2 b$

PPM 
${ }^{1} \mathrm{H} \mathrm{NMR}\left(400 \mathrm{MHz}, \mathrm{CDCl}_{3}\right)$

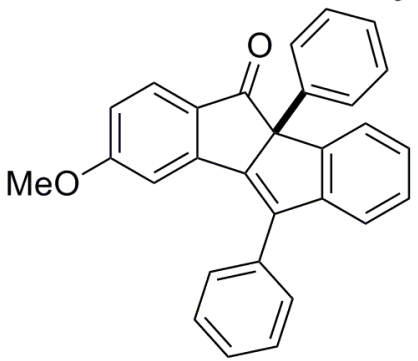

2c

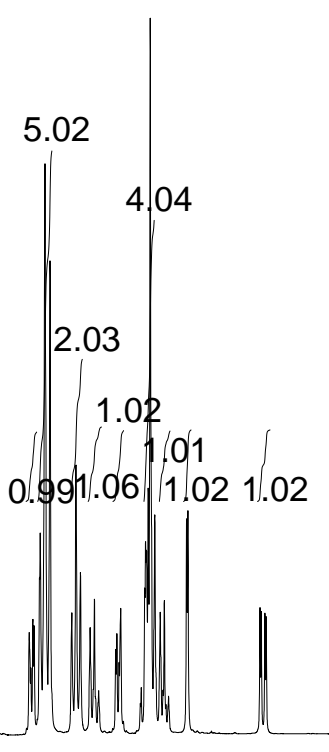


${ }^{13} \mathrm{C} \mathrm{NMR}\left(100 \mathrm{MHz}, \mathrm{CDCl}_{3}\right)$

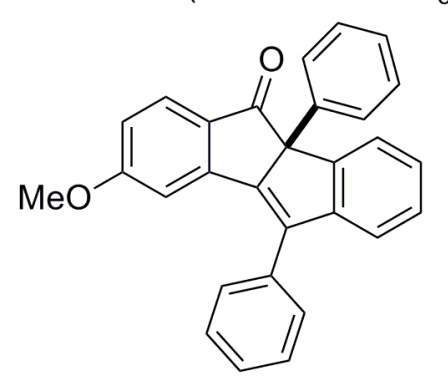

2c 
${ }^{1} \mathrm{H}$ NMR $\left(400 \mathrm{MHz}, \mathrm{CDCl}_{3}\right)$

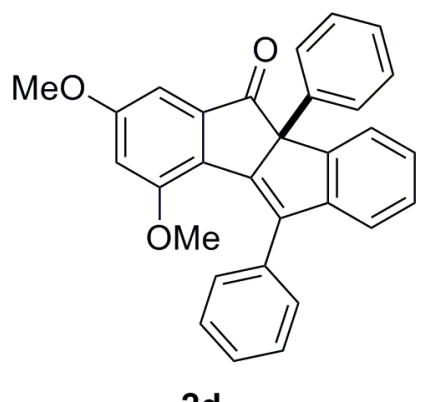

2d

6.05

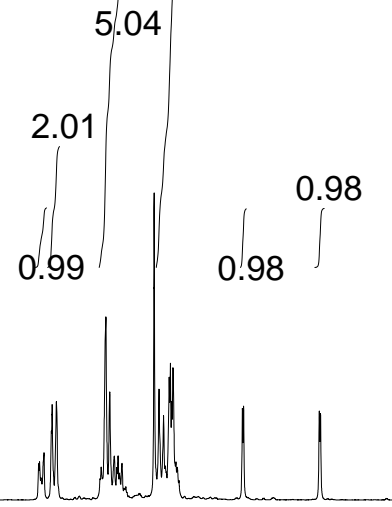

PPM 


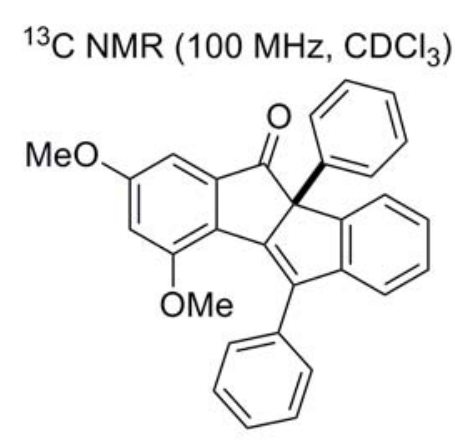

2d 

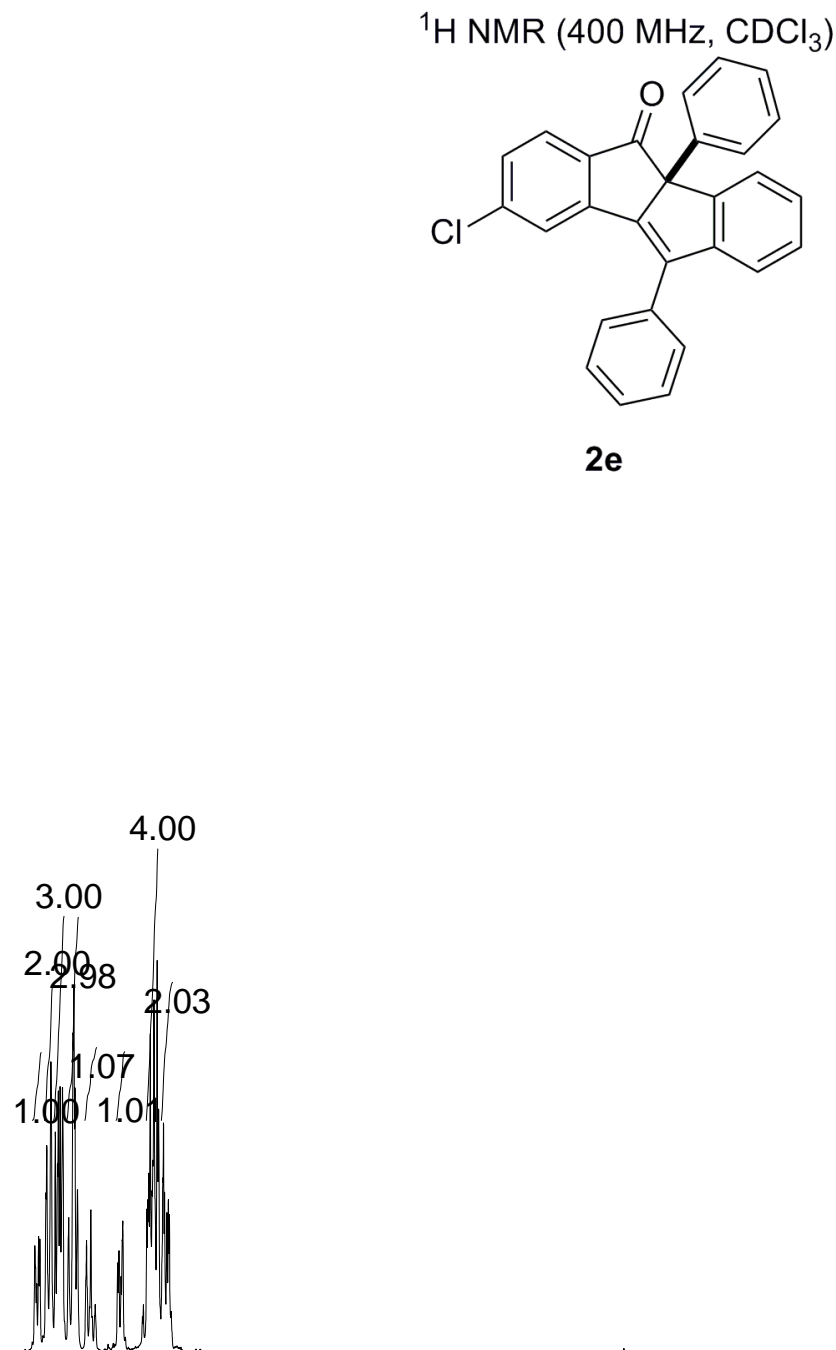


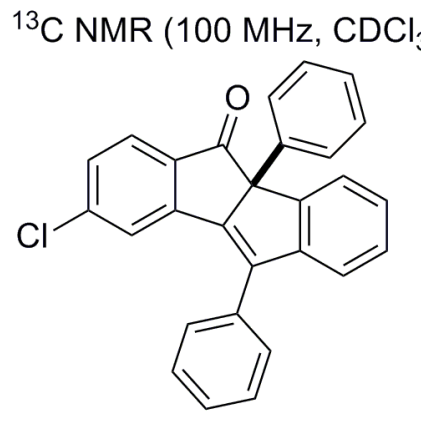

$2 e$

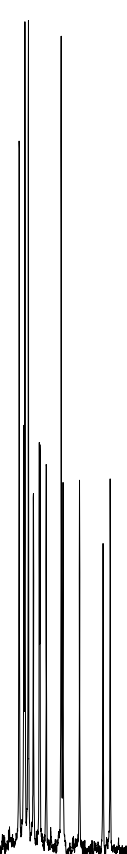




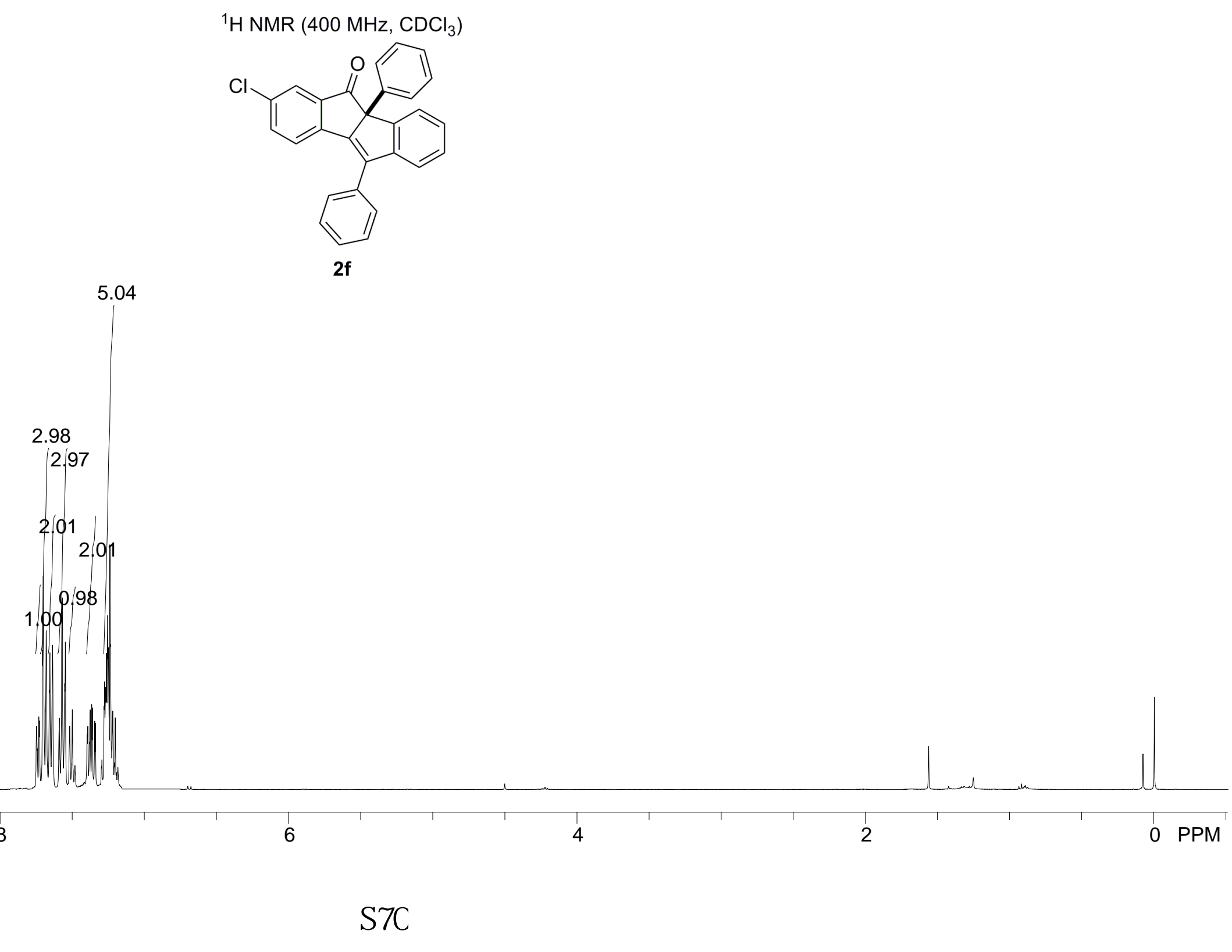




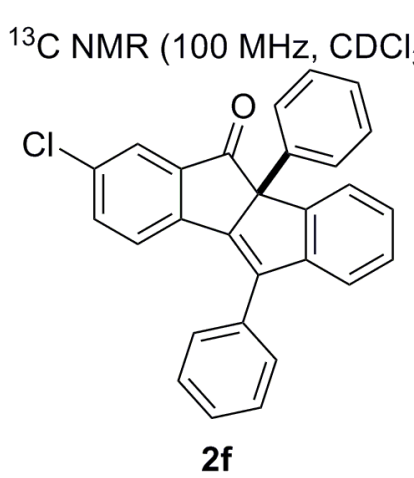


${ }^{1} \mathrm{H} \mathrm{NMR}\left(400 \mathrm{MHz}, \mathrm{CDCl}_{3}\right)$

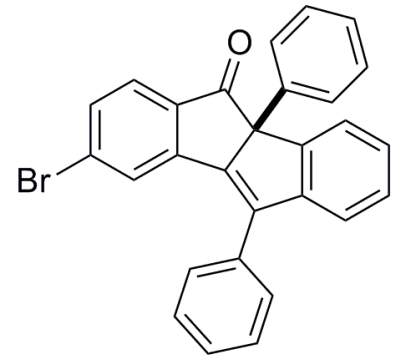

$2 \mathrm{~g}$

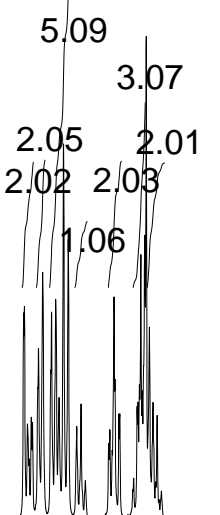




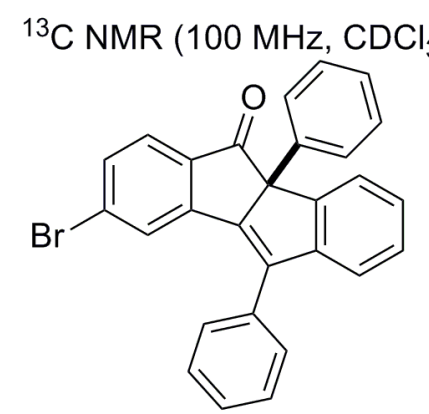

$2 g$ 


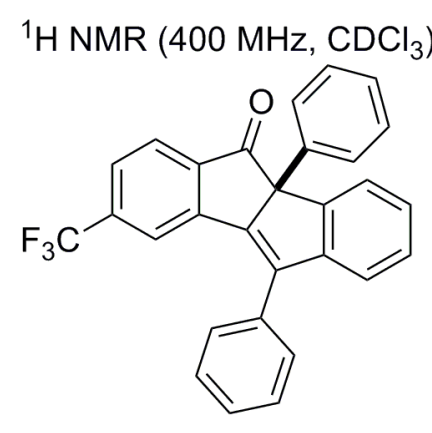

2h

5.07

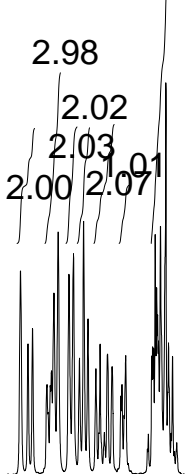


${ }^{13} \mathrm{CNMR}\left(100 \mathrm{MHz}, \mathrm{CDCl}_{3}\right)$

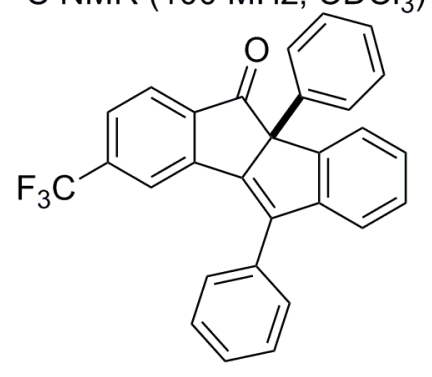

$2 \mathrm{~h}$ 
${ }^{1} \mathrm{H} \mathrm{NMR}\left(400 \mathrm{MHz}, \mathrm{CDCl}_{3}\right)$

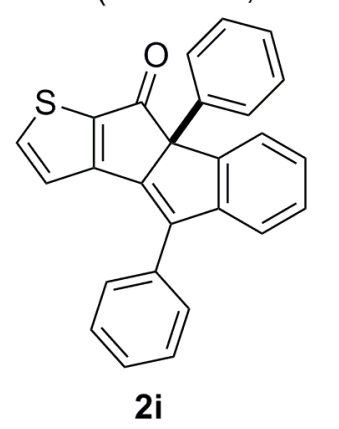

6.02

5.13

4.08 
${ }^{13} \mathrm{C}$ NMR $\left(100 \mathrm{MHz}, \mathrm{CDCl}_{3}\right)$

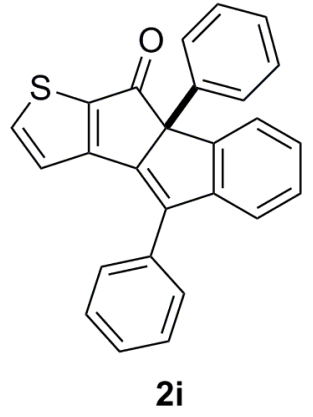


${ }^{1} \mathrm{H}$ NMR $\left(400 \mathrm{MHz}, \mathrm{CDCl}_{3}\right)$

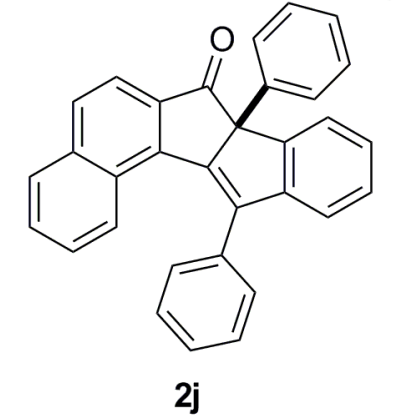




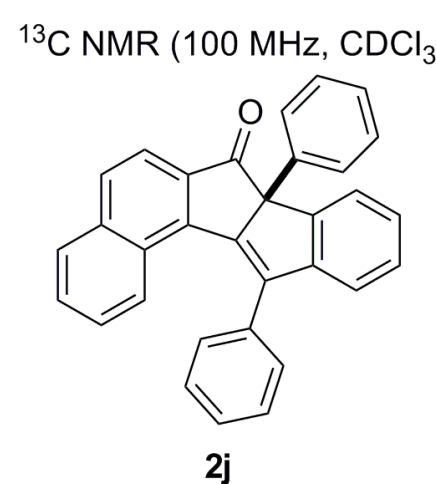


${ }^{1} \mathrm{H} \mathrm{NMR}\left(400 \mathrm{MHz}, \mathrm{CDCl}_{3}\right)$

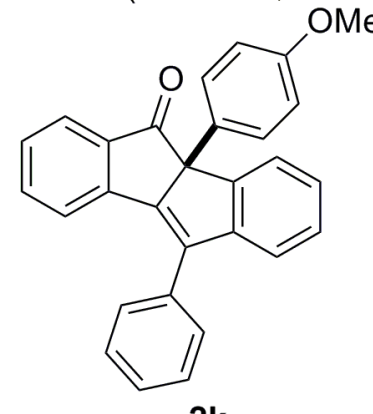

$2 k$

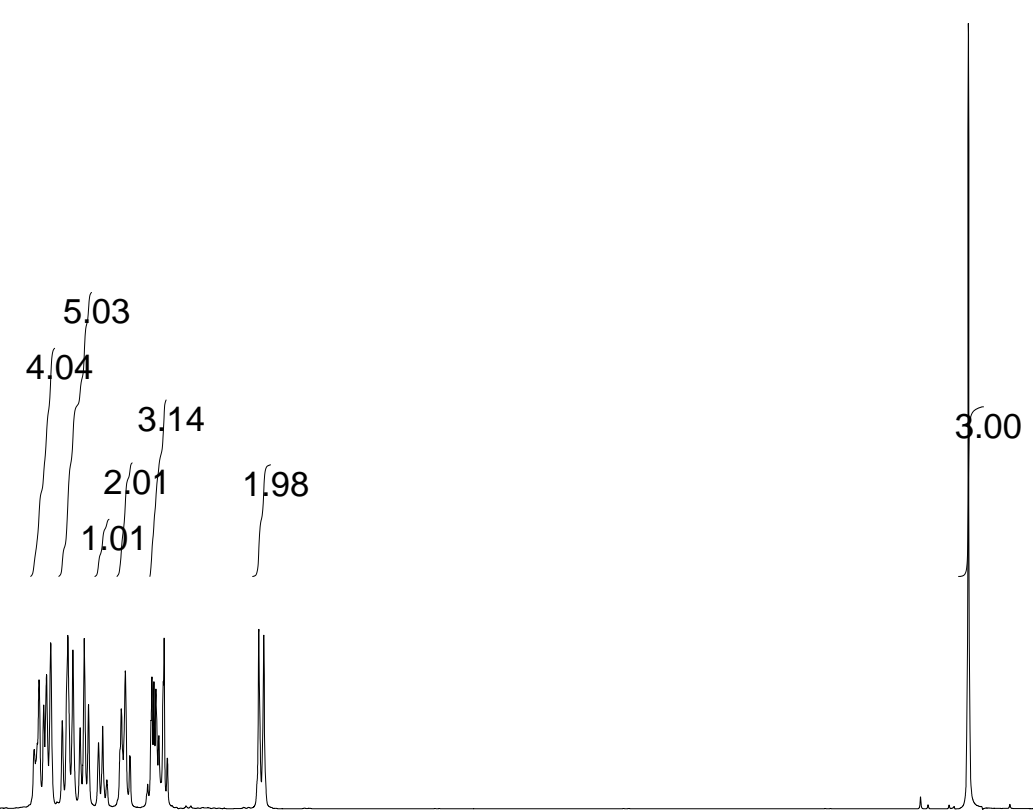




\section{${ }^{1} \mathrm{H} \mathrm{NMR}\left(400 \mathrm{MHz}, \mathrm{CDCl}_{3}\right)$}

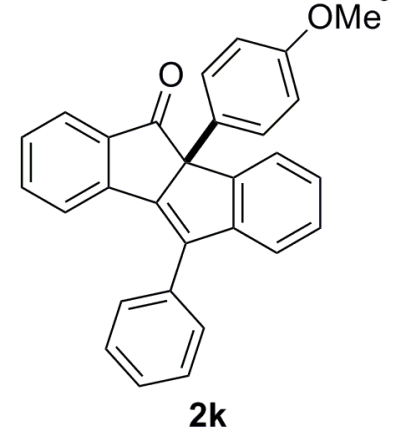


${ }^{1} \mathrm{H}$ NMR $\left(400 \mathrm{MHz}, \mathrm{CDCl}_{3}\right)$

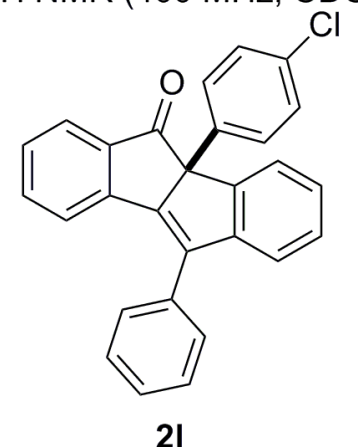

PPM 
${ }^{13} \mathrm{C}$ NMR $\left(100 \mathrm{MHz}, \mathrm{CDCl}_{3}\right)$

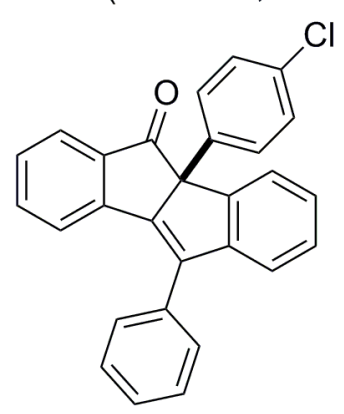

2I 


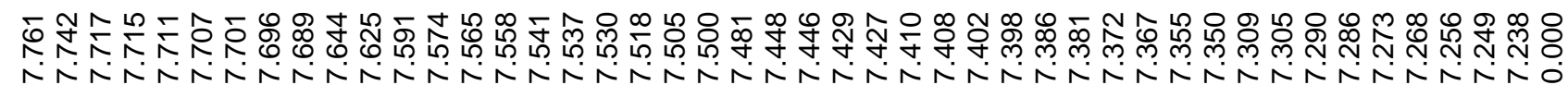

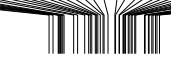

${ }^{1} \mathrm{H} \mathrm{NMR}\left(400 \mathrm{MHz}, \mathrm{CDCl}_{3}\right)$

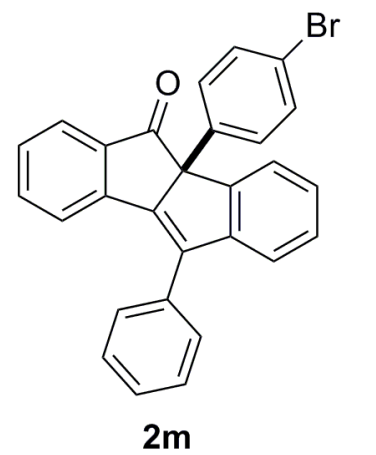

4.98

3.004 .025 
${ }^{13} \mathrm{C} \mathrm{NMR}\left(100 \mathrm{MHz}, \mathrm{CDCl}_{3}\right)$

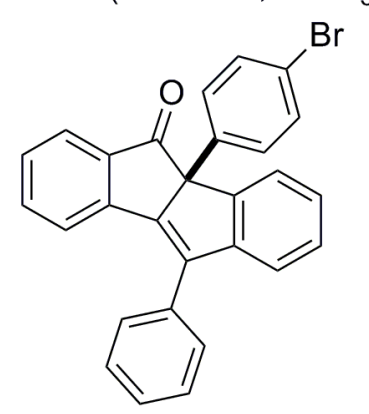

$2 m$

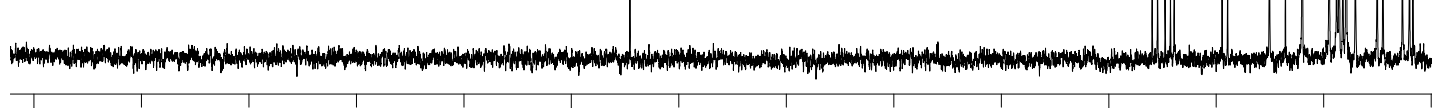

200

PPM 


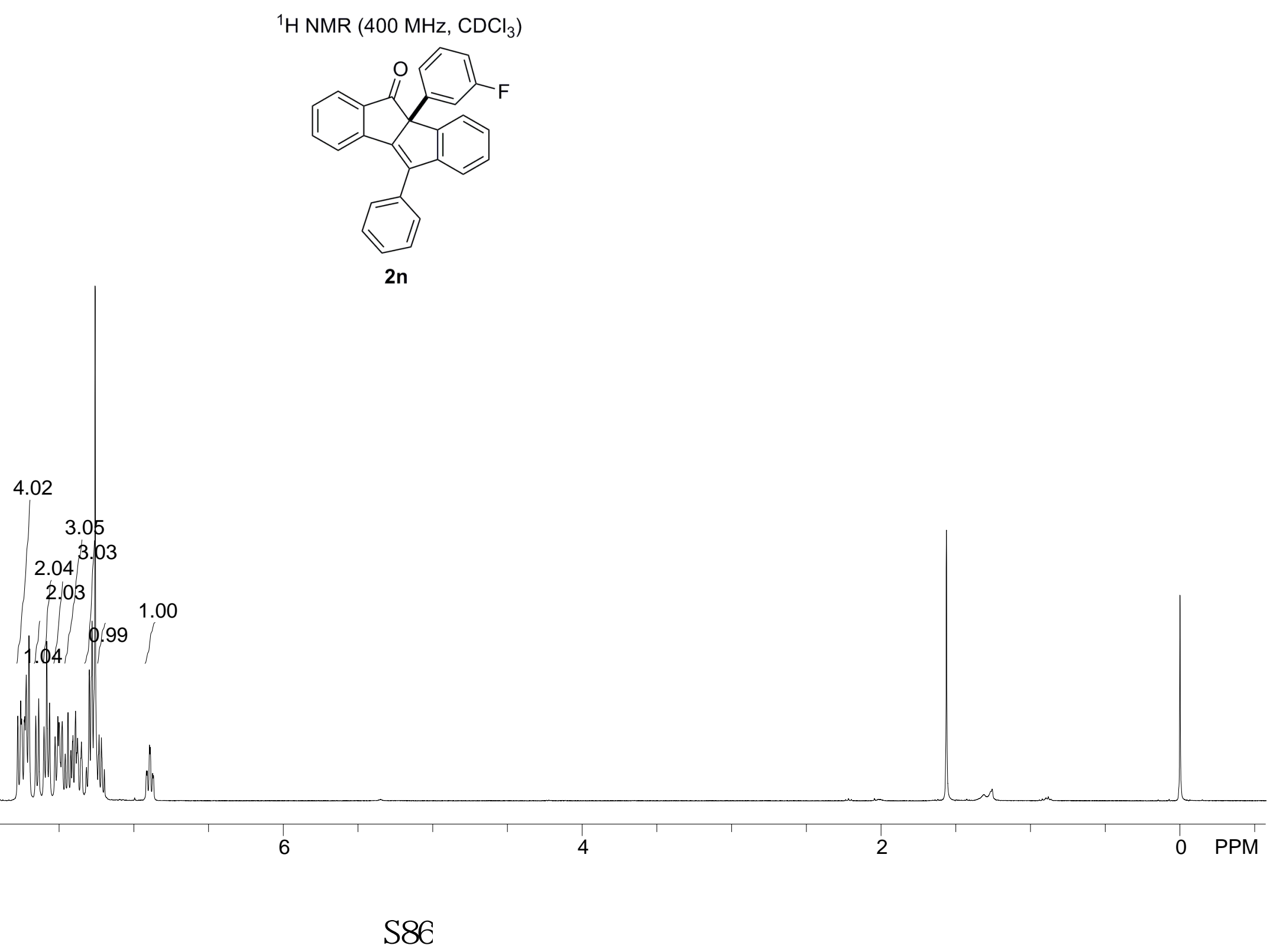




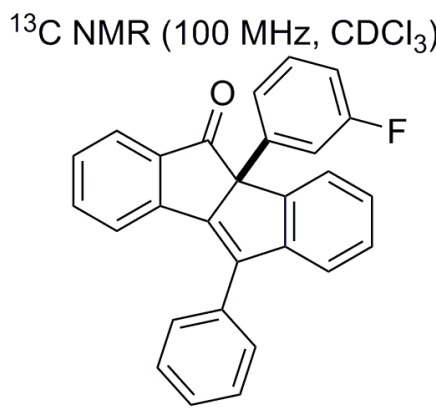

2n 
${ }^{1} \mathrm{H}$ NMR $\left(400 \mathrm{MHz}, \mathrm{CDCl}_{3}\right)$

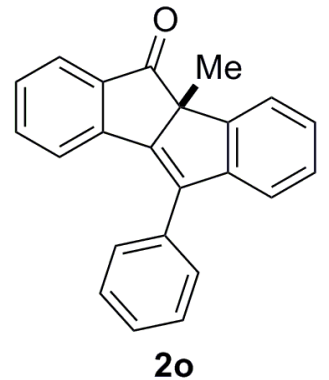

20

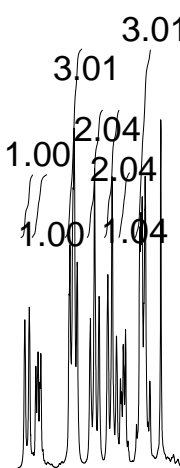


${ }^{13} \mathrm{C}$ NMR $\left(100 \mathrm{MHz}, \mathrm{CDCl}_{3}\right)$

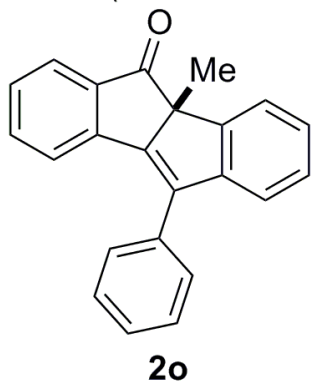




\section{${ }^{1} \mathrm{H} \mathrm{NMR}\left(400 \mathrm{MHz}, \mathrm{CDCl}_{3}\right)$}
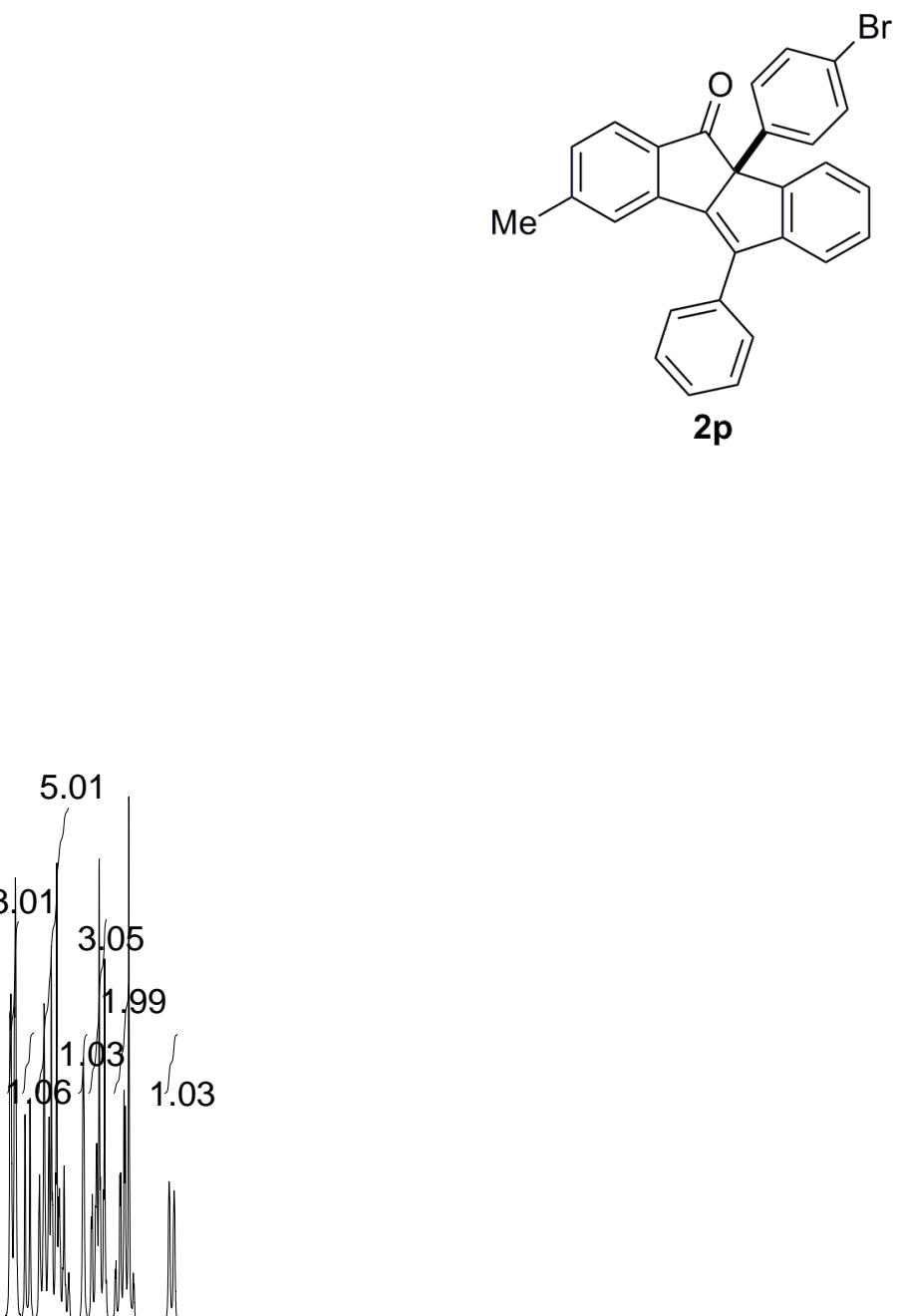
${ }^{13} \mathrm{C}$ NMR $\left(100 \mathrm{MHz}, \mathrm{CDCl}_{3}\right)$

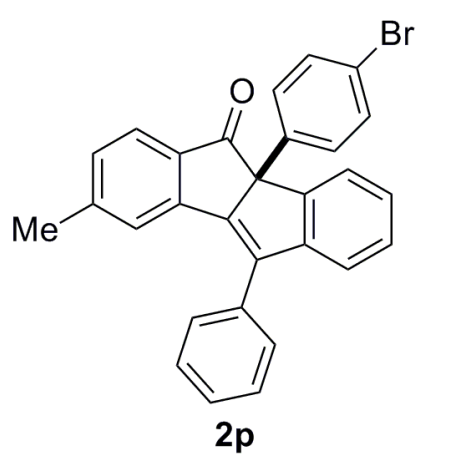




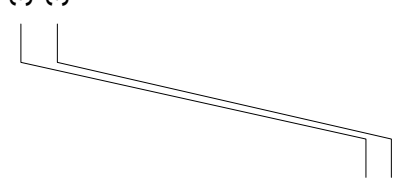

${ }^{1} \mathrm{H}$ NMR $\left(400 \mathrm{MHz}, \mathrm{CDCl}_{3}\right)$
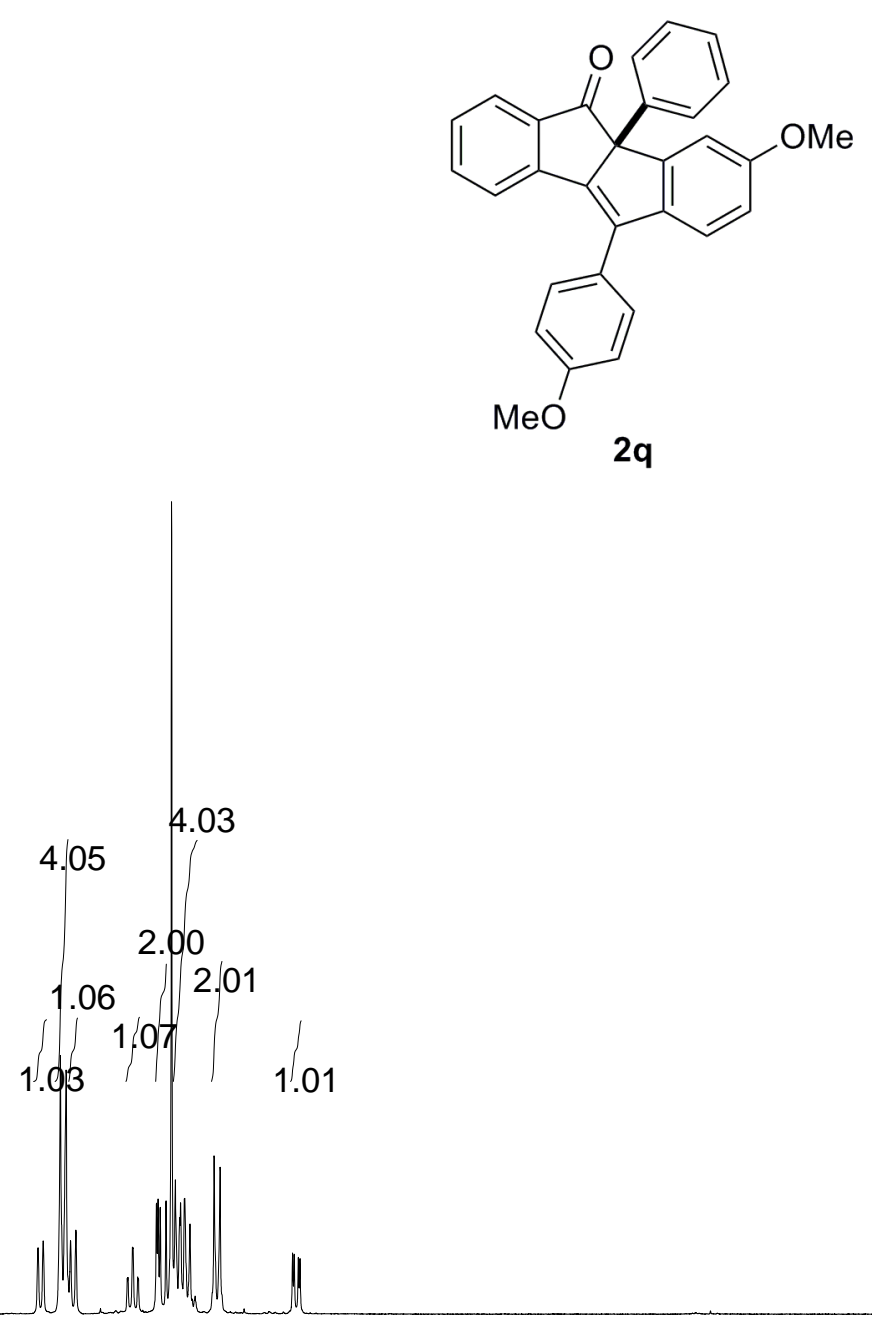

3.00

2. 97 


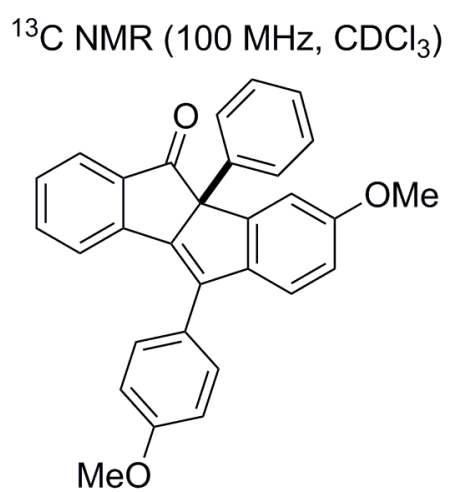

$2 q$ 
${ }^{1} \mathrm{H}$ NMR $\left(400 \mathrm{MHz}, \mathrm{CDCl}_{3}\right)$

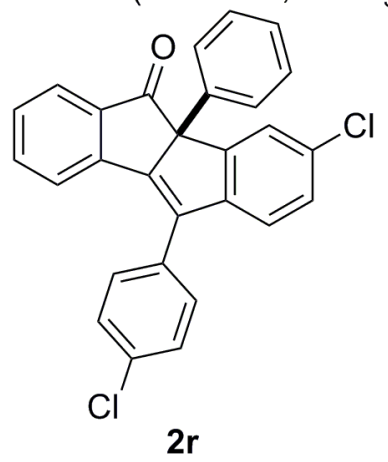

7.01

6.13 
${ }^{13} \mathrm{C}$ NMR $\left(100 \mathrm{MHz}, \mathrm{CDCl}_{3}\right)$

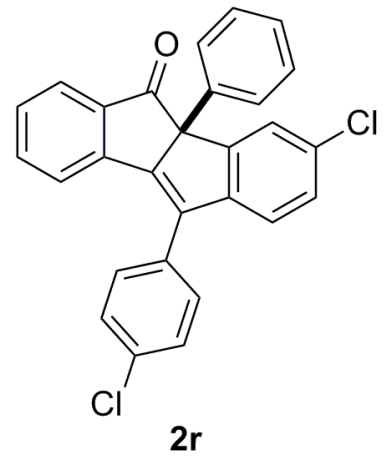


${ }^{1} \mathrm{H}$ NMR $\left(400 \mathrm{MHz}, \mathrm{CDCl}_{3}\right)$

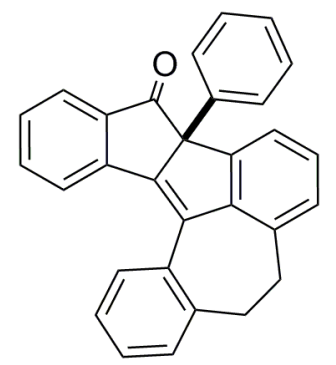

2s

$2.97 \quad 3.05$

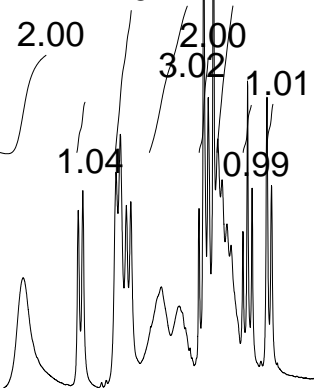

3.99 
${ }^{13} \mathrm{C}$ NMR $\left(100 \mathrm{MHz}, \mathrm{CDCl}_{3}\right)$

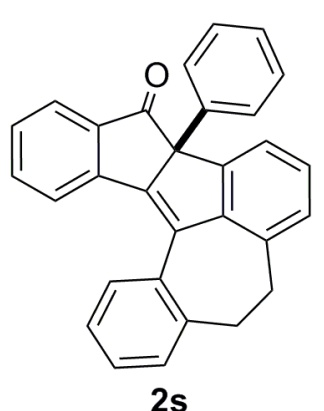



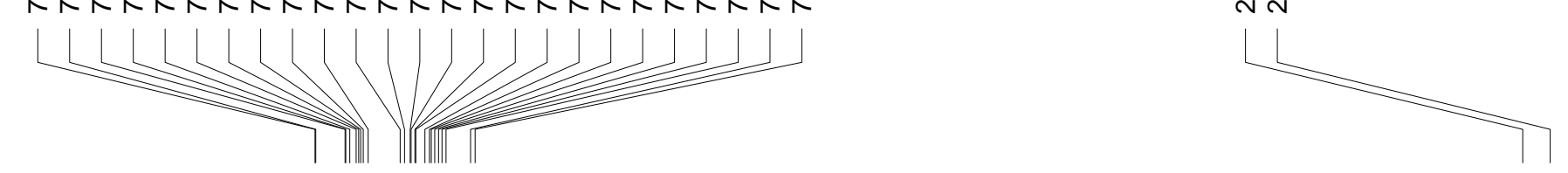

${ }^{1} \mathrm{H}$ NMR $\left(400 \mathrm{MHz}, \mathrm{CDCl}_{3}\right)$

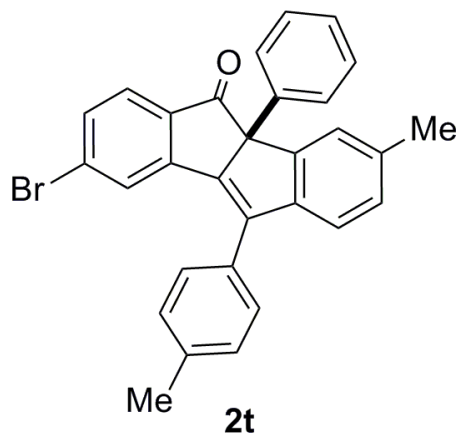

\section{$5.06 \quad 5.04$}


${ }^{13} \mathrm{C}$ NMR (100 MHz, $\mathrm{CDCl}_{3}$ )

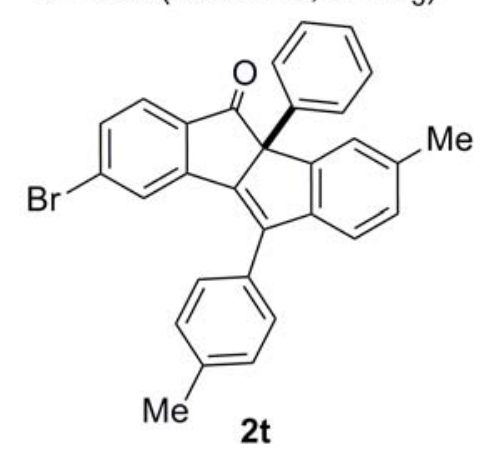



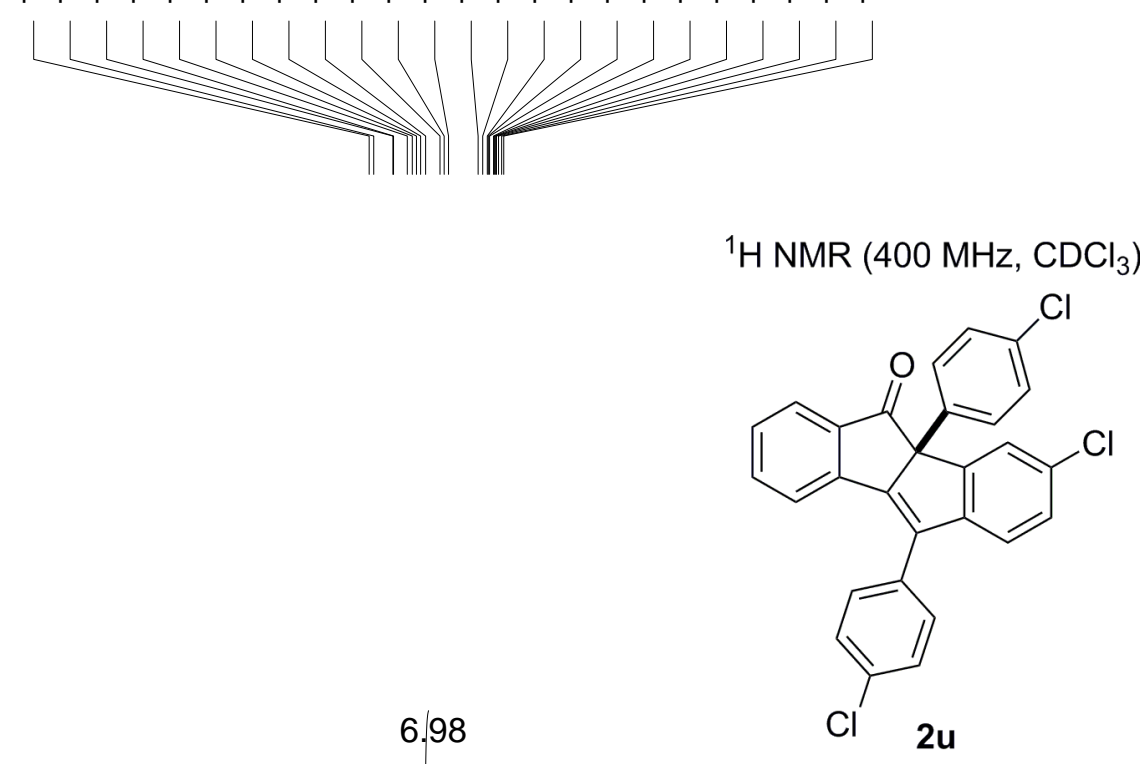
${ }^{13} \mathrm{C}$ NMR (100 MHz, $\left.\mathrm{CDCl}_{3}\right)$

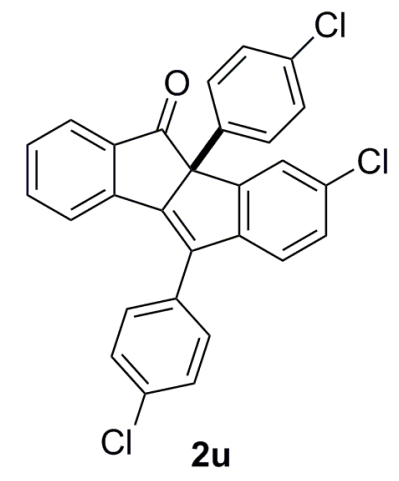


${ }^{1} \mathrm{H}$ NMR $\left(400 \mathrm{MHz}, \mathrm{CDCl}_{3}\right)$

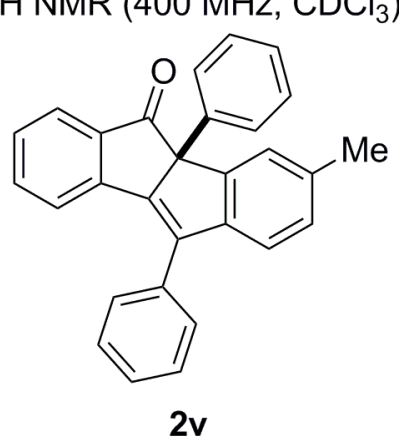

2v

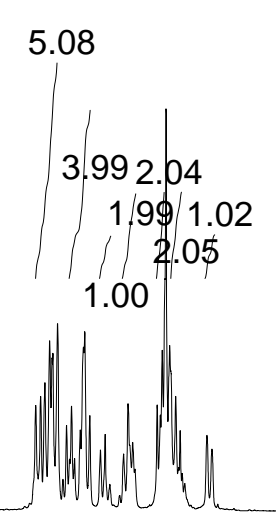

PPM 
$\left.{ }^{13} \mathrm{C} \mathrm{NMR} \mathrm{(100} \mathrm{MHz,} \mathrm{CDCl}_{3}\right)$

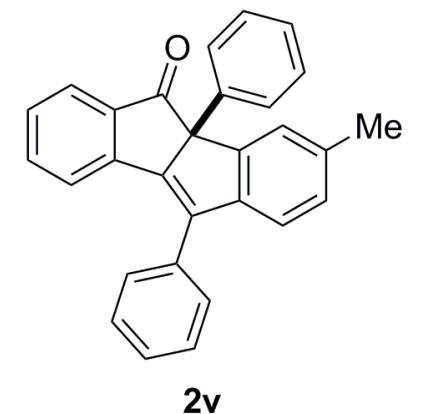

2v

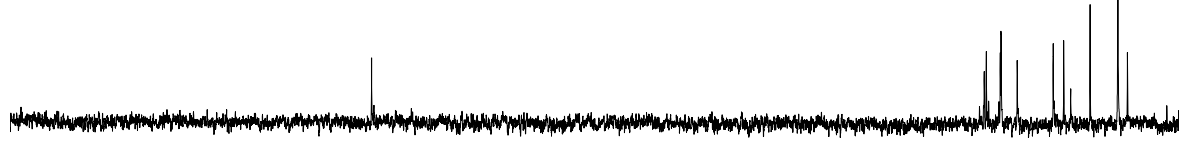


${ }^{1} \mathrm{H} \mathrm{NMR}\left(400 \mathrm{MHz}, \mathrm{CDCl}_{3}\right)$

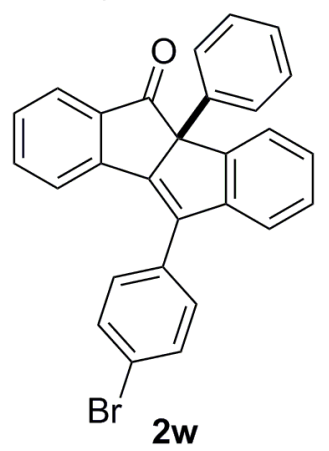

$4.03 \quad 4.06$

2.98

2.03

$2.00 \quad 0.97$ 
${ }^{13} \mathrm{C}$ NMR $\left(100 \mathrm{MHz}, \mathrm{CDCl}_{3}\right)$

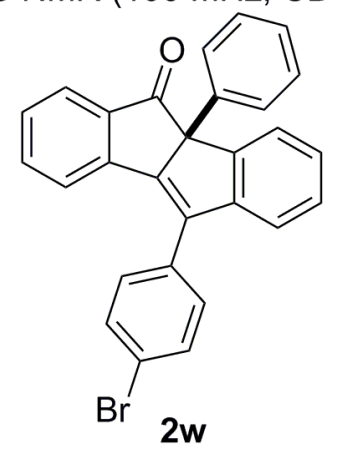




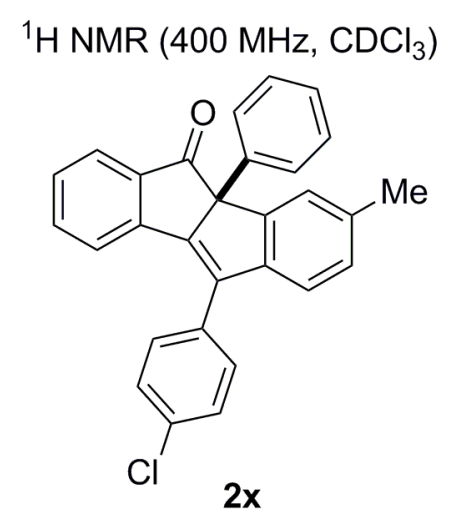

5.03

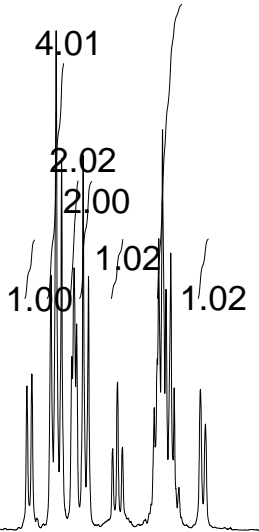


${ }^{13} \mathrm{C}$ NMR $\left(100 \mathrm{MHz}, \mathrm{CDCl}_{3}\right)$

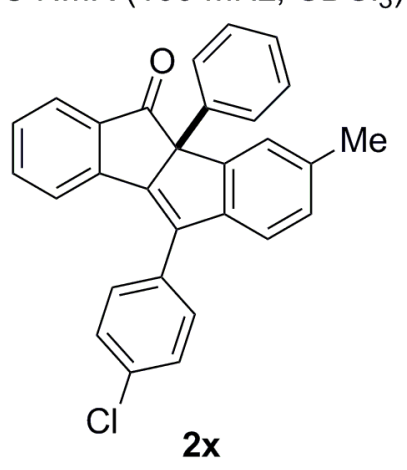


${ }^{1} \mathrm{H} \mathrm{NMR}\left(400 \mathrm{MHz}, \mathrm{CDCl}_{3}\right)$

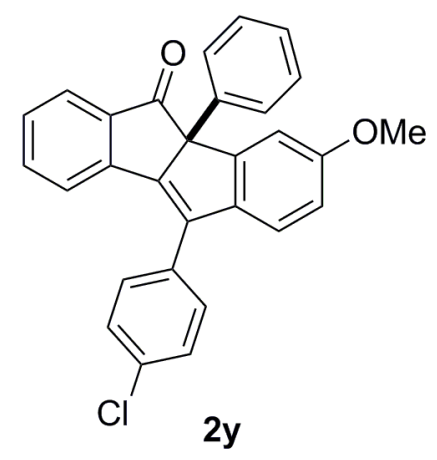

5.01

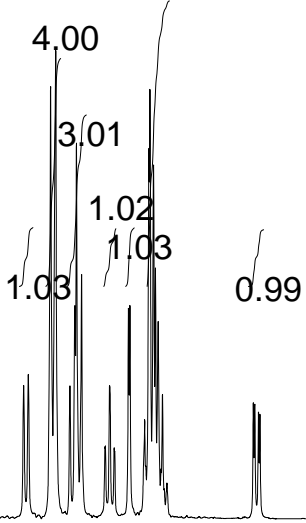




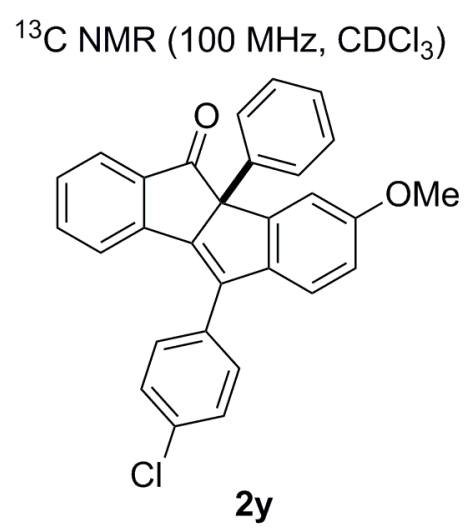




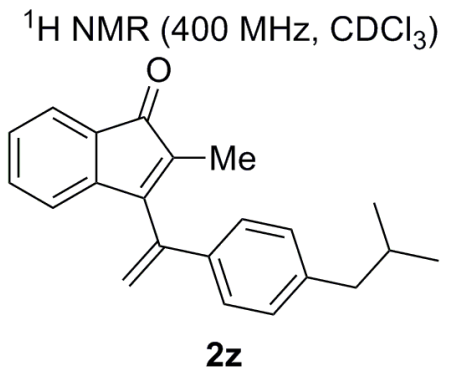

$2 z$
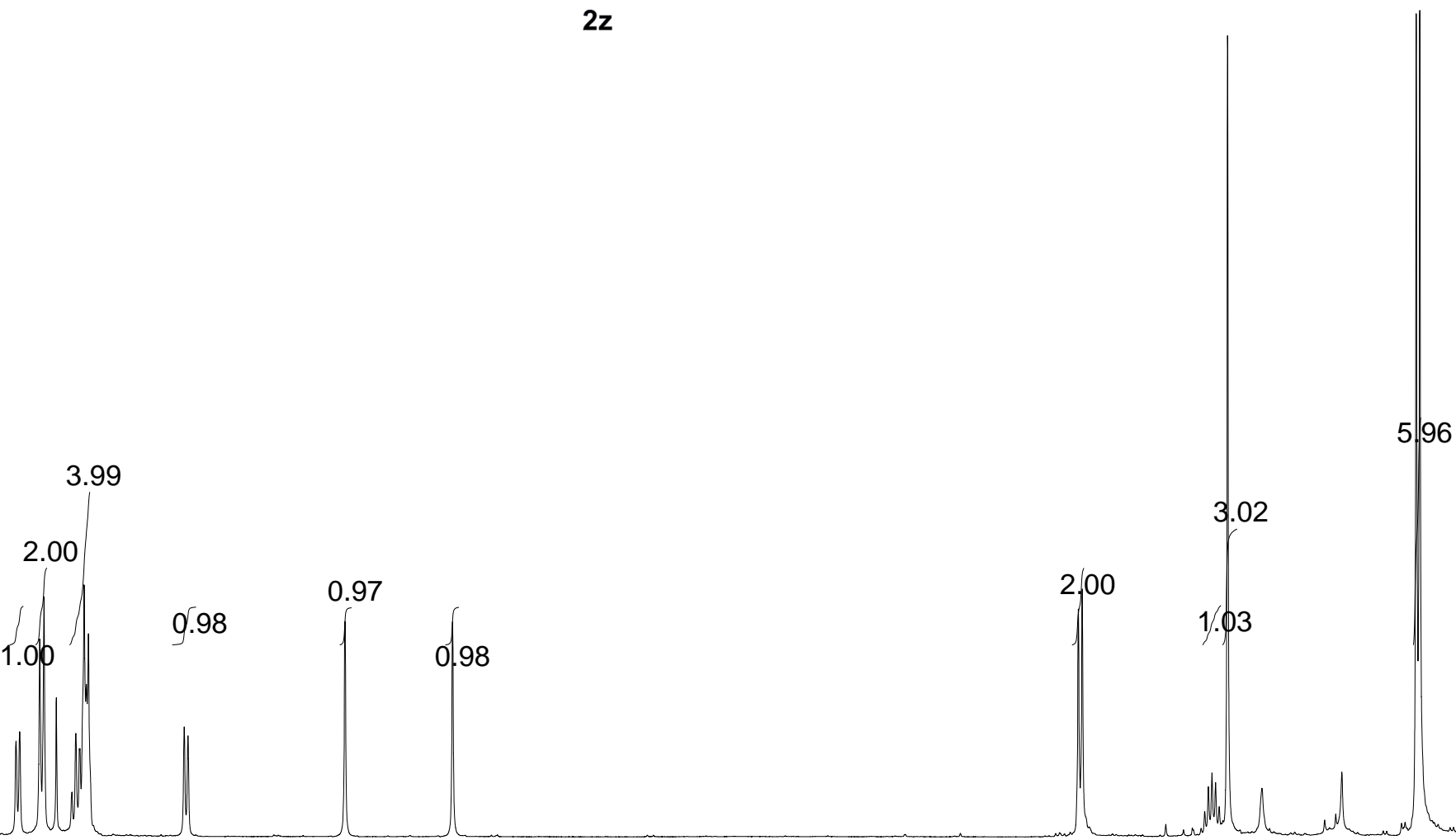


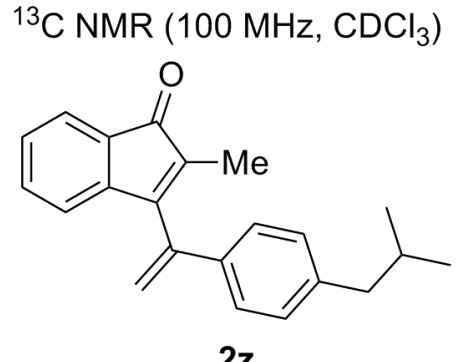

$2 z$ 


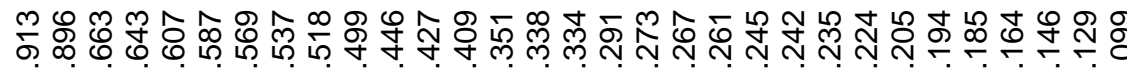
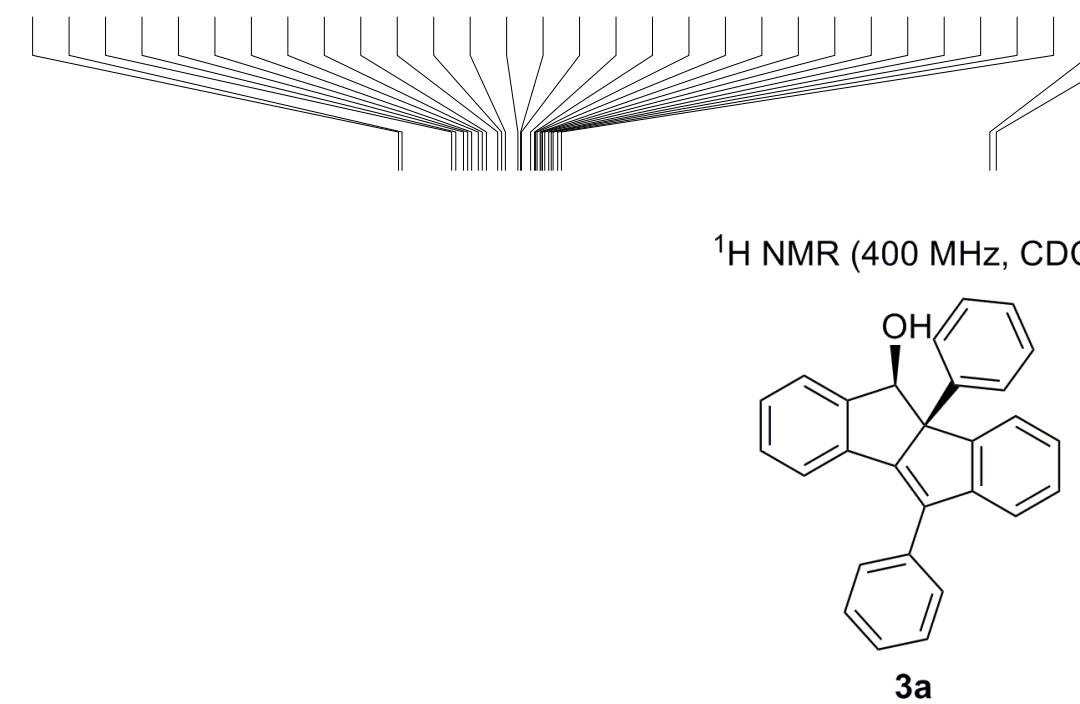

$3 a$

${ }^{1} \mathrm{H}$ NMR $\left(400 \mathrm{MHz}, \mathrm{CDCl}_{3}\right)$

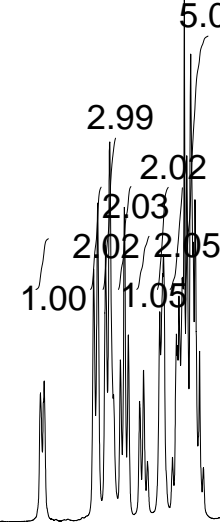


${ }^{13} \mathrm{C}$ NMR $\left(100 \mathrm{MHz}, \mathrm{CDCl}_{3}\right)$

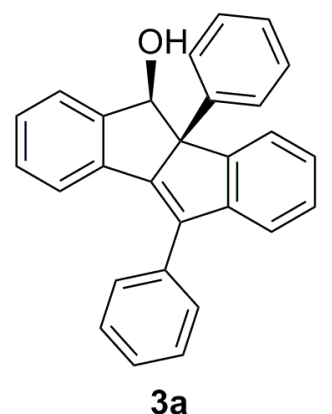


${ }^{1} \mathrm{H}$ NMR $\left(400 \mathrm{MHz}, \mathrm{CDCl}_{3}\right)$

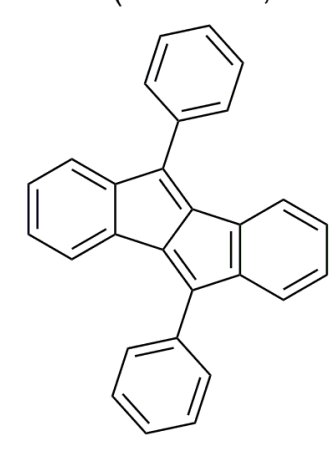

$4 a$ 


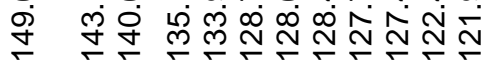

${ }^{13} \mathrm{C} \mathrm{NMR}\left(100 \mathrm{MHz}, \mathrm{CDCl}_{3}\right)$

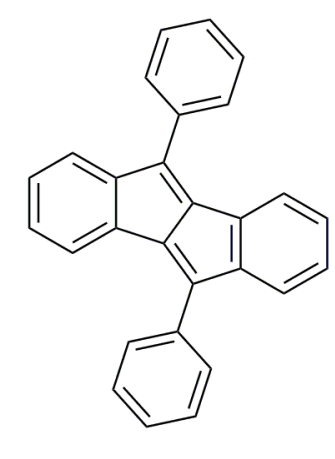




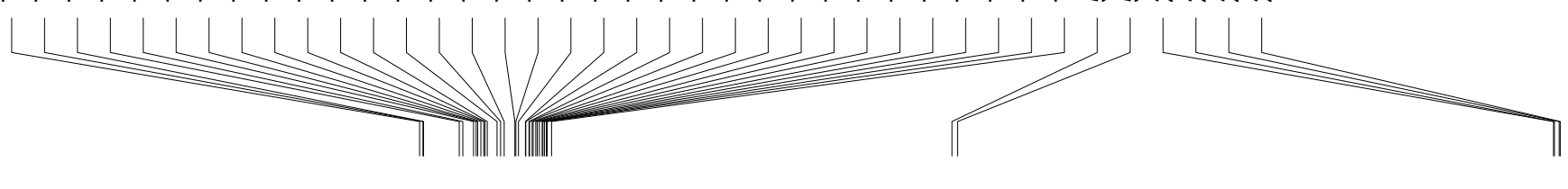

${ }^{1} \mathrm{H}$ NMR $\left(400 \mathrm{MHz}, \mathrm{CDCl}_{3}\right)$
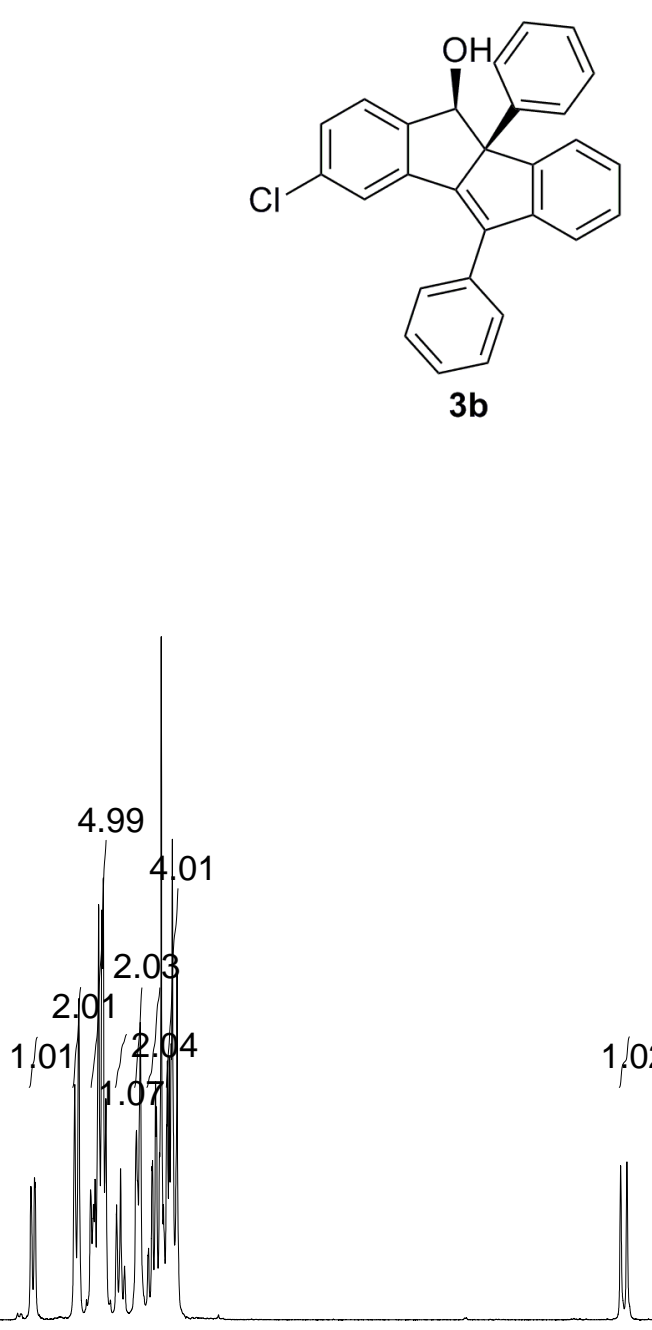

1.02

1.03 
${ }^{13} \mathrm{C}$ NMR (100 MHz, CDCl $)$<smiles>O[C@H]1c2ccc(Cl)cc2C2=C(c3ccccc3)c3ccccc3C2(c2ccccc2)[C@H]1c1ccccc1</smiles>

$3 b$ 


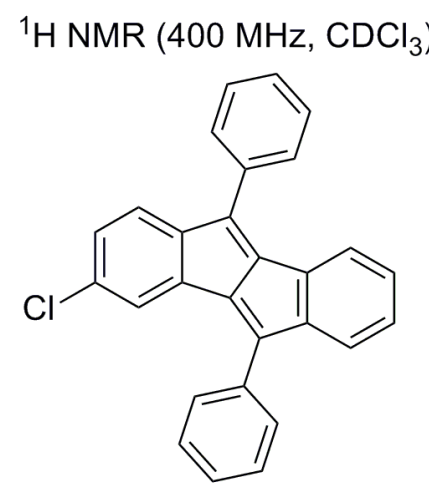

4b

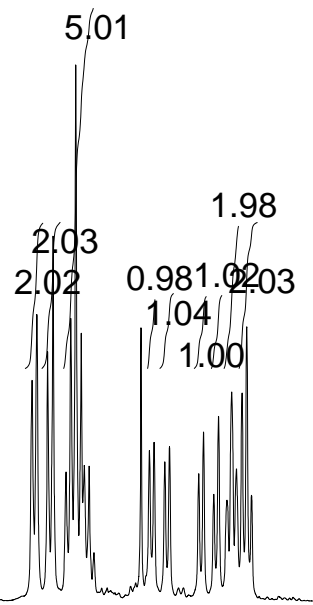

PPM 


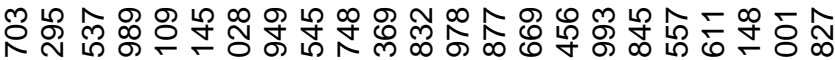

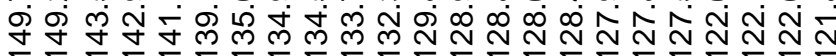

${ }^{13} \mathrm{C}$ NMR $\left(100 \mathrm{MHz}, \mathrm{CDCl}_{3}\right)$

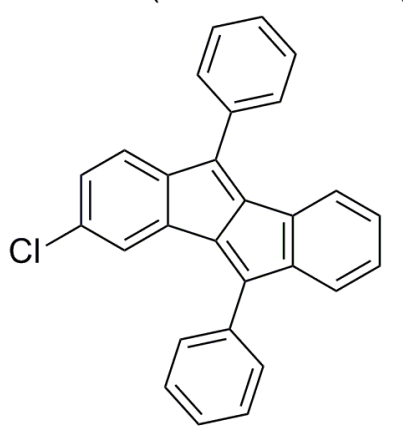

4b 
${ }^{1} \mathrm{H} \mathrm{NMR}\left(400 \mathrm{MHz}, \mathrm{CDCl}_{3}\right)$

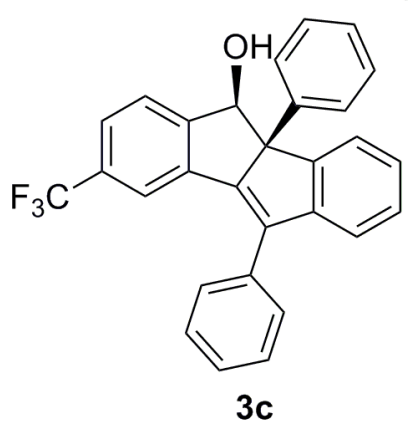

\subsection{5}

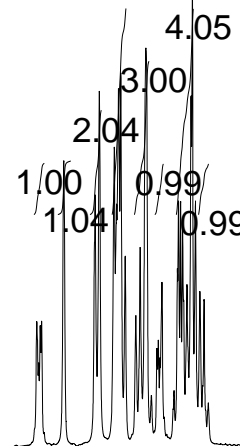




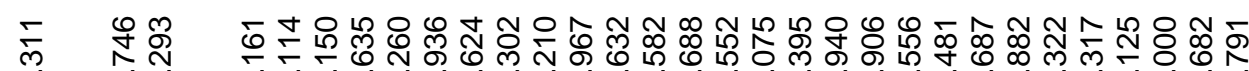

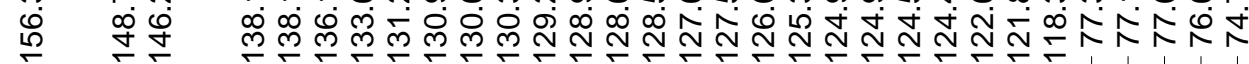

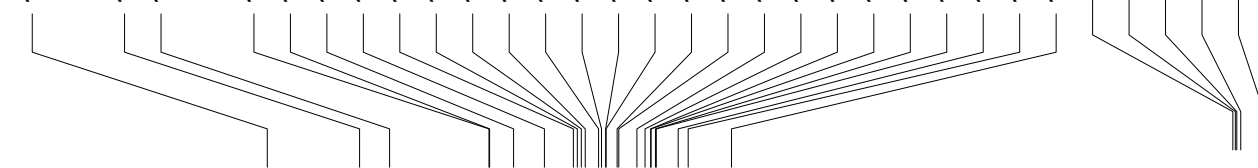

${ }^{13} \mathrm{C}$ NMR $\left(100 \mathrm{MHz}, \mathrm{CDCl}_{3}\right)$

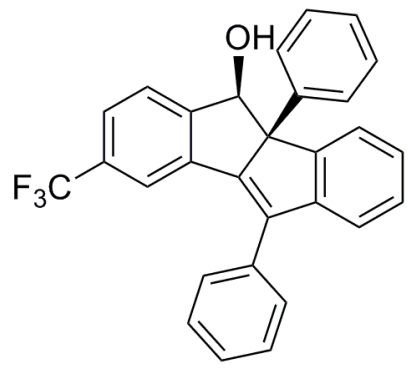

$3 c$ 
${ }^{1} \mathrm{H}$ NMR $\left(400 \mathrm{MHz}, \mathrm{CDCl}_{3}\right)$

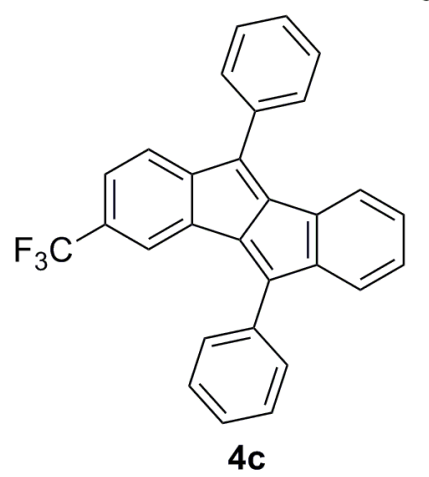

4c 


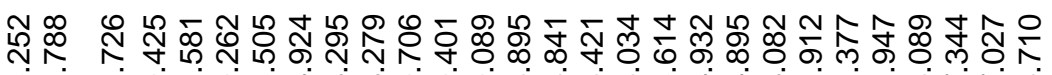

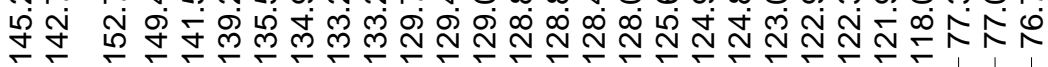

${ }^{13} \mathrm{C}$ NMR $\left(100 \mathrm{MHz}, \mathrm{CDCl}_{3}\right)$

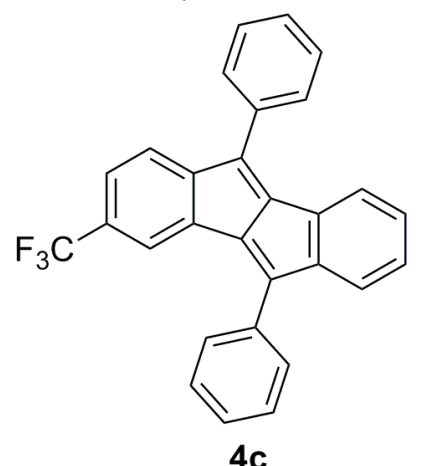

4c 
${ }^{1} \mathrm{H} \mathrm{NMR}\left(400 \mathrm{MHz}, \mathrm{CDCl}_{3}\right)$
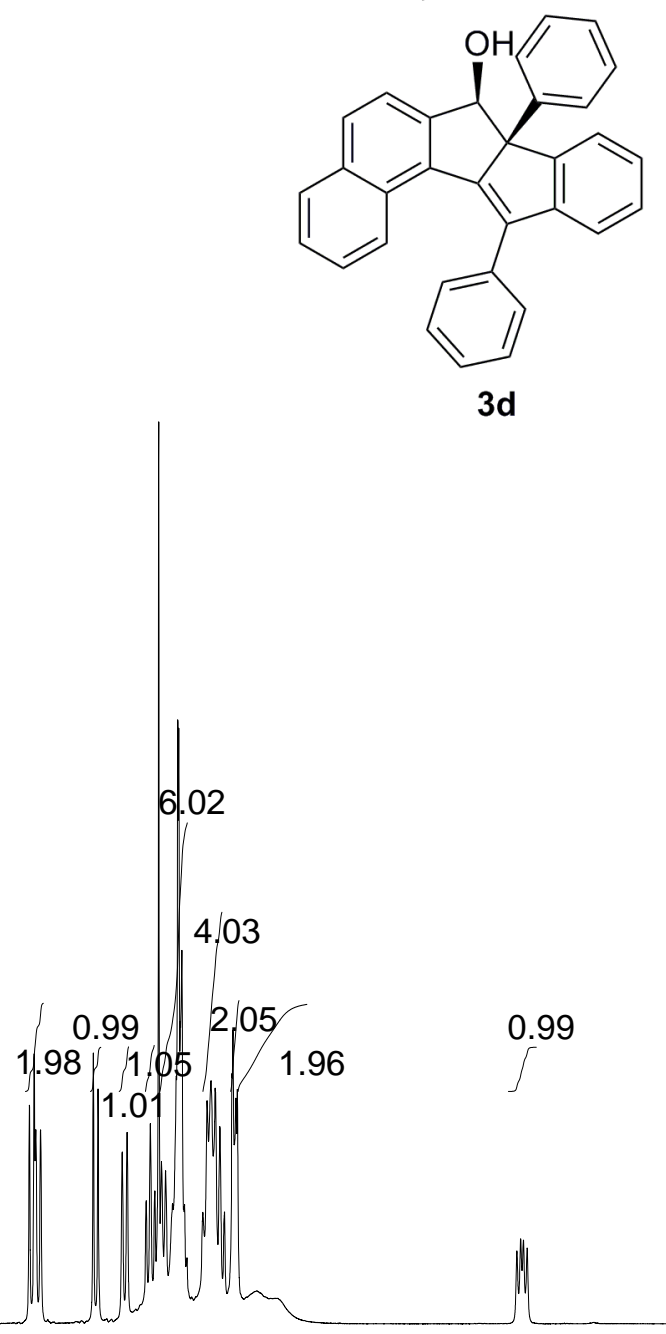

$0.99 \quad 0.99$ 
${ }^{13} \mathrm{C} \mathrm{NMR}\left(100 \mathrm{MHz}, \mathrm{CDCl}_{3}\right)$<smiles>OC1C2=C(c3ccccc3)C(c3ccccc3)(c3ccccc32)c2c1ccc1ccccc21</smiles>

3d

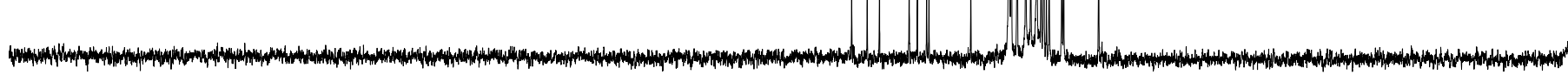




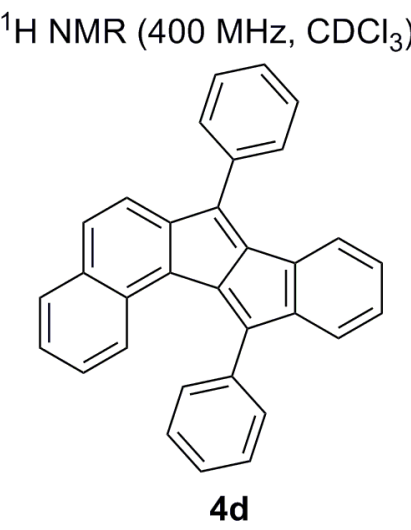

7.96

3.98

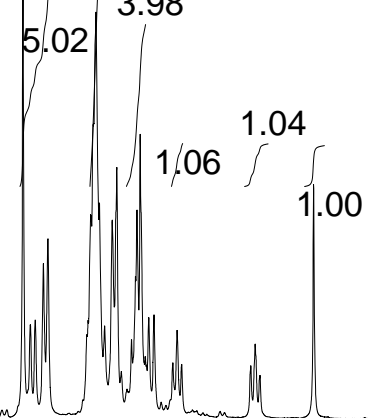




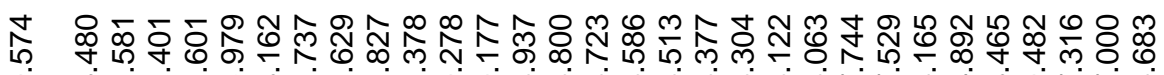

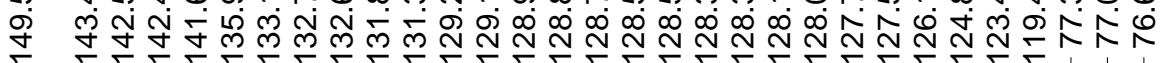

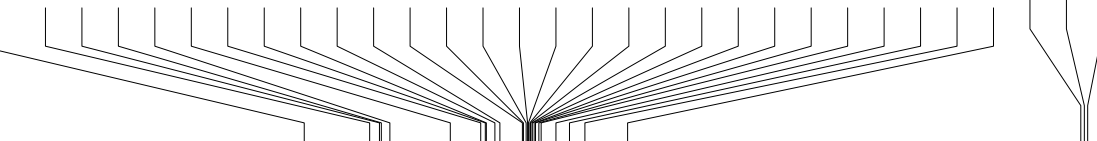

${ }^{13} \mathrm{C}$ NMR $\left(100 \mathrm{MHz}, \mathrm{CDCl}_{3}\right)$

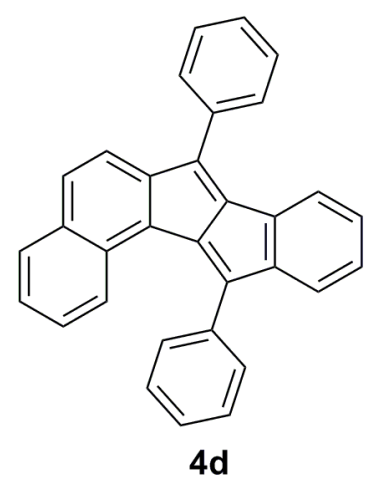


${ }^{1} \mathrm{H}$ NMR $\left(400 \mathrm{MHz}, \mathrm{CDCl}_{3}\right)$

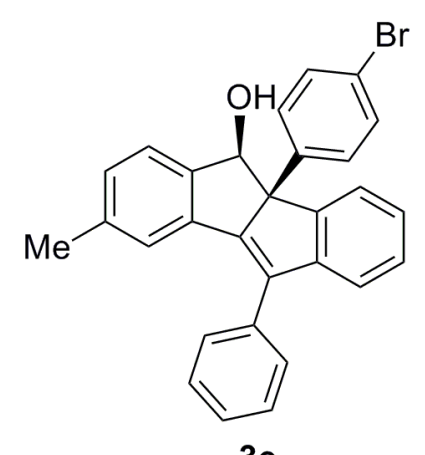

$3 e$

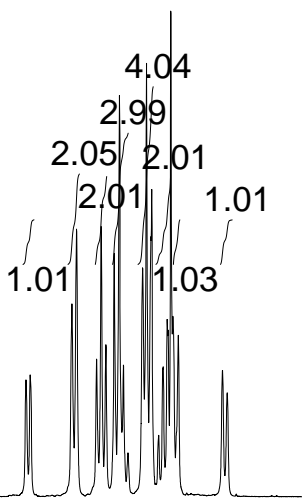

1.00

2.99 
${ }^{13} \mathrm{C} \mathrm{NMR}\left(100 \mathrm{MHz}, \mathrm{CDCl}_{3}\right)$

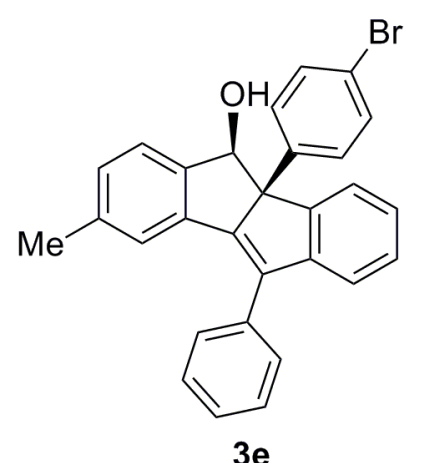

$3 e$ 
${ }^{1} \mathrm{H}$ NMR $\left(400 \mathrm{MHz}, \mathrm{CDCl}_{3}\right)$

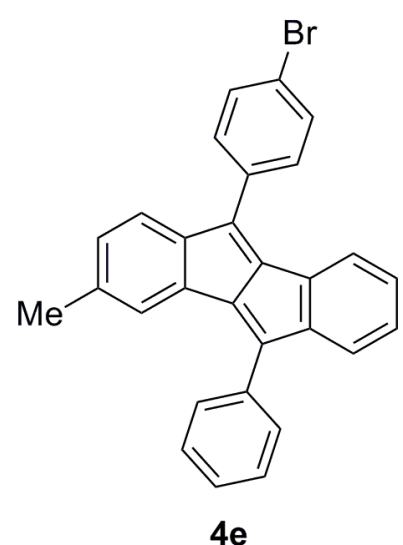

$4 e$

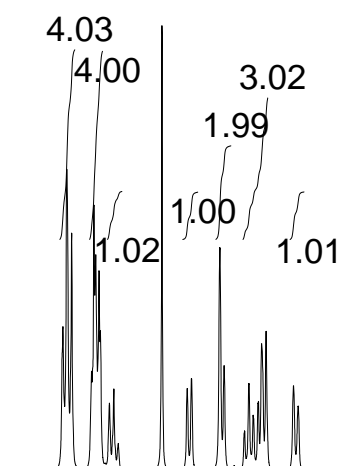

3.00 


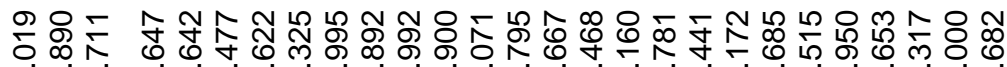

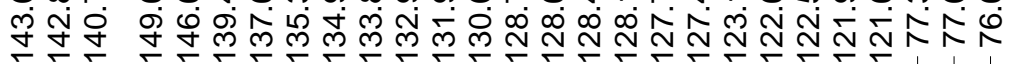

${ }^{13} \mathrm{C}$ NMR $\left(100 \mathrm{MHz}, \mathrm{CDCl}_{3}\right.$ )

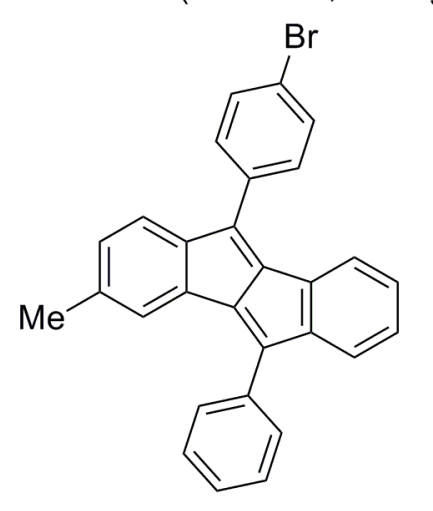

$4 e$ 


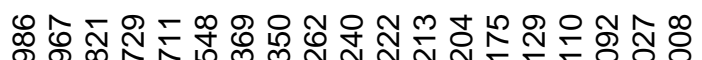

ำ

hin Mrim

${ }^{1} \mathrm{H} \mathrm{NMR}\left(400 \mathrm{MHz}, \mathrm{CDCl}_{3}\right)$

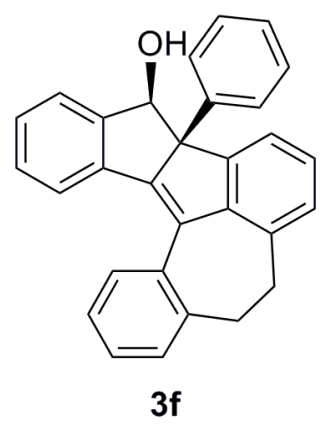

9.00

PPM 
${ }^{13} \mathrm{C}$ NMR $\left(100 \mathrm{MHz}, \mathrm{CDCl}_{3}\right)$<smiles>O[C@H]1c2ccccc2C2=C3c4ccccc4CCc4cccc(c43)C2(c2ccccc2)[C@H]1O</smiles> 

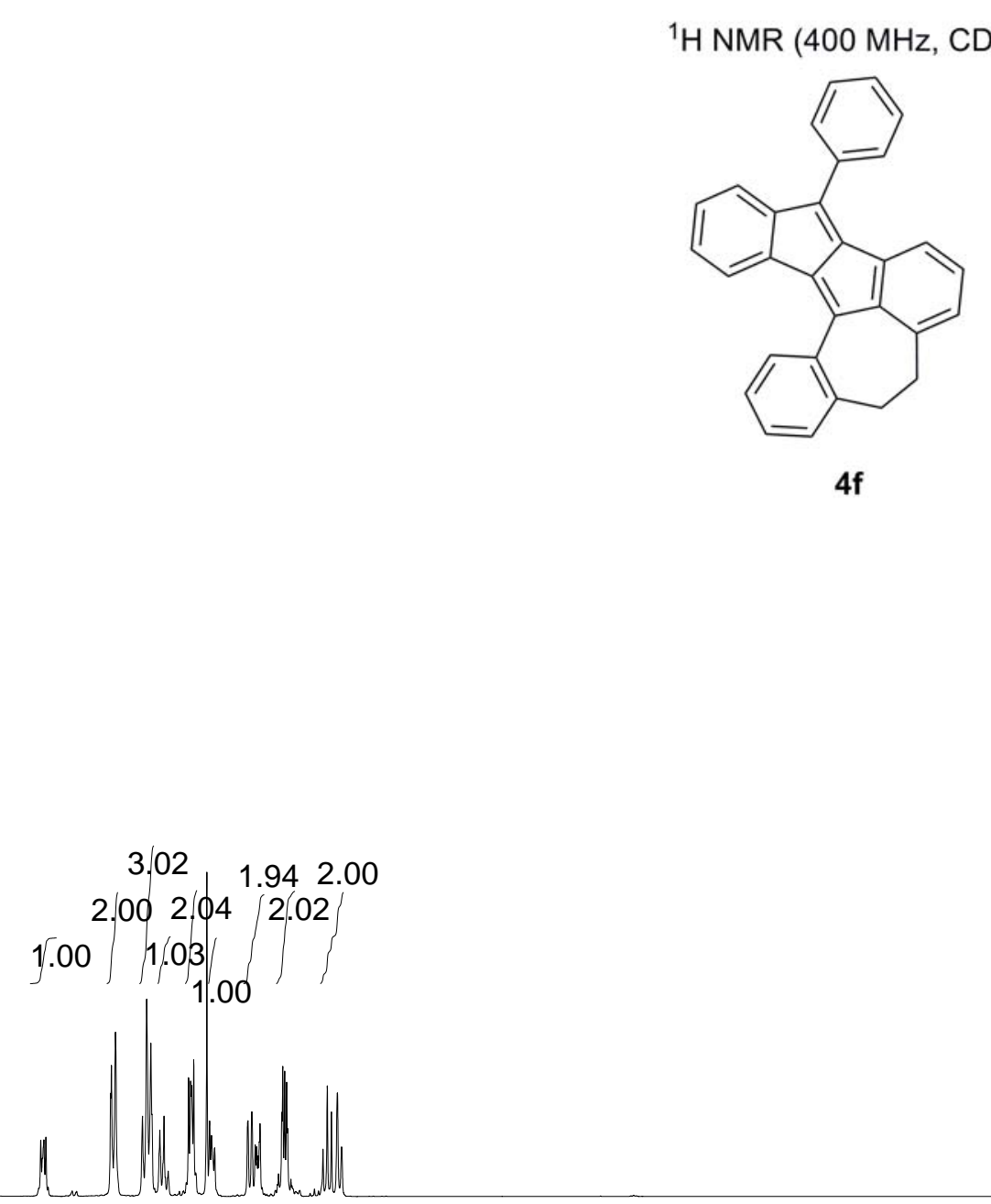
${ }^{13} \mathrm{C} \mathrm{NMR}\left(100 \mathrm{MHz}, \mathrm{CDCl}_{3}\right)$

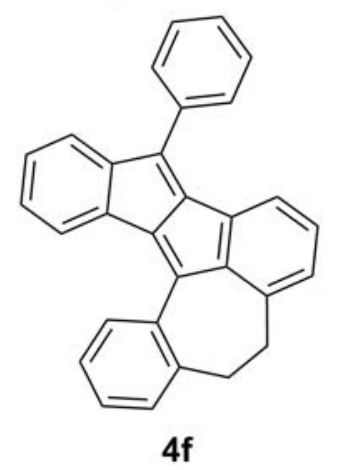



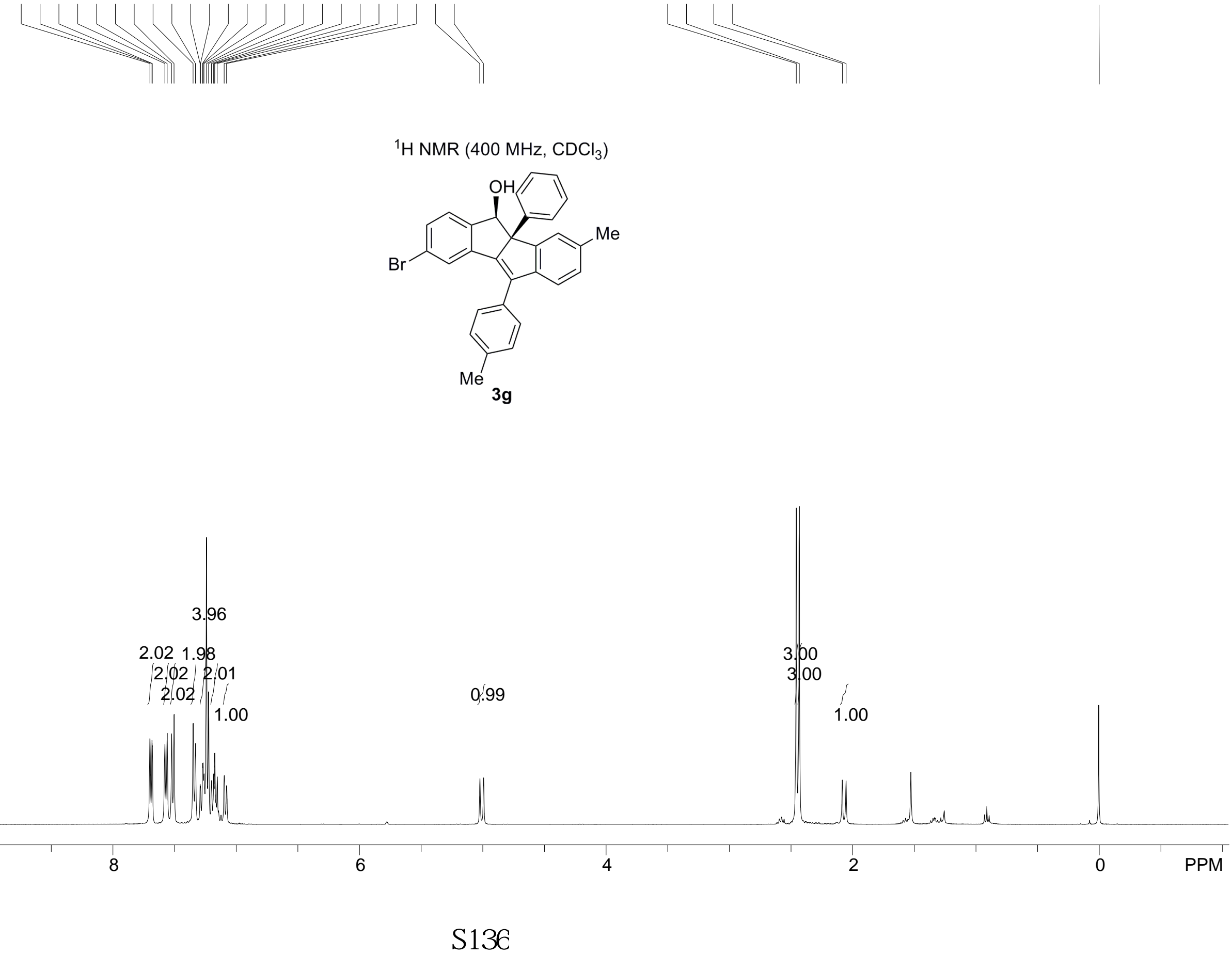
${ }^{13} \mathrm{C}$ NMR $\left(100 \mathrm{MHz}, \mathrm{CDCl}_{3}\right)$

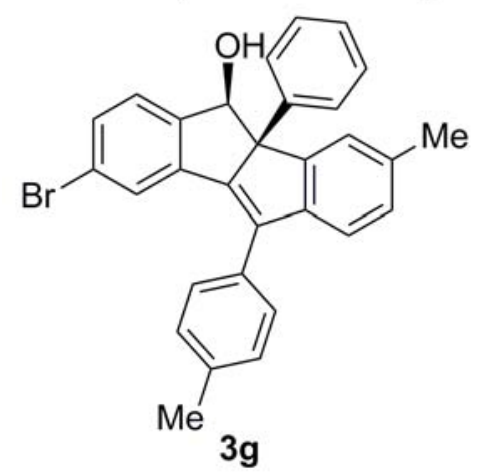


${ }^{1} \mathrm{H}$ NMR (400 MHz, $\left.\mathrm{CDCl}_{3}\right)$

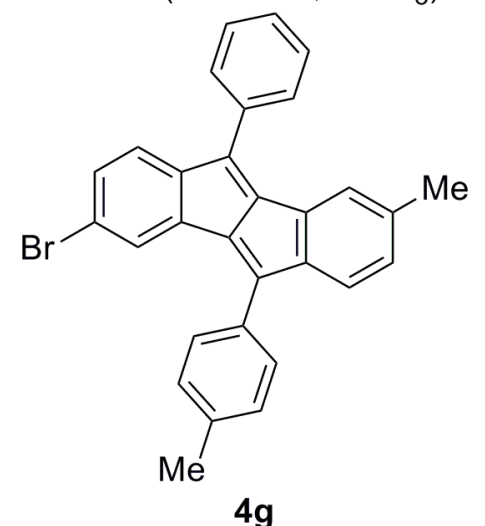

$4 \mathrm{~g}$

\subsection{3}

2.982 .02

$2.055_{1.00}^{1.02}$

1.00

$1.03 \quad 1.03$ 
${ }^{13} \mathrm{C}$ NMR $\left(100 \mathrm{MHz}, \mathrm{CDCl}_{3}\right)$

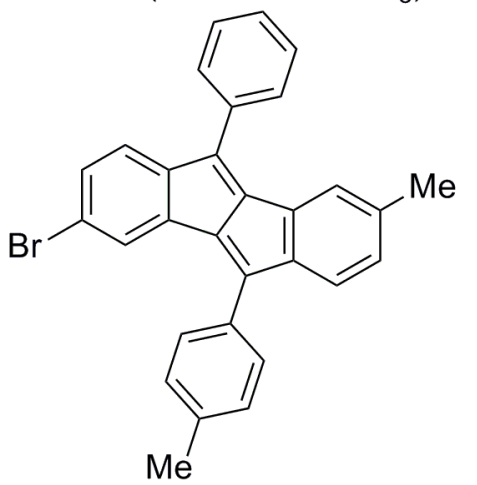




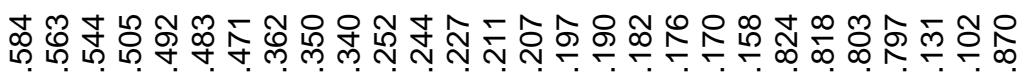

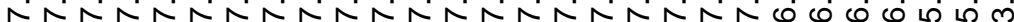
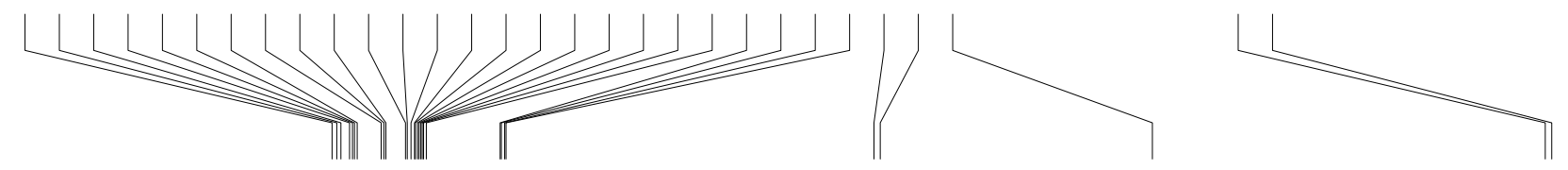

${ }^{1} \mathrm{H}$ NMR $\left(400 \mathrm{MHz}, \mathrm{CDCl}_{3}\right)$

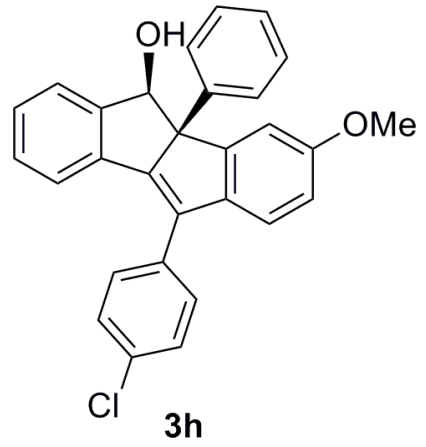


${ }^{13} \mathrm{C}$ NMR $\left(100 \mathrm{MHz}, \mathrm{CDCl}_{3}\right)$

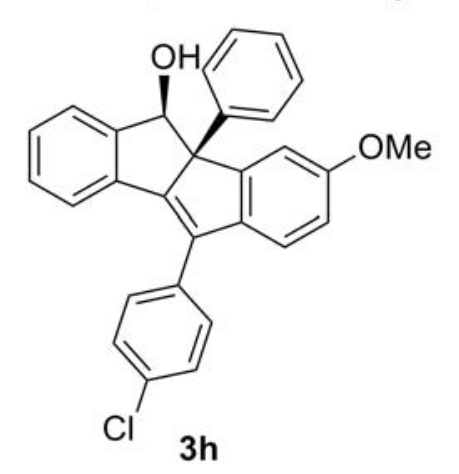




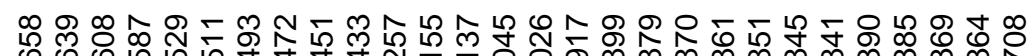
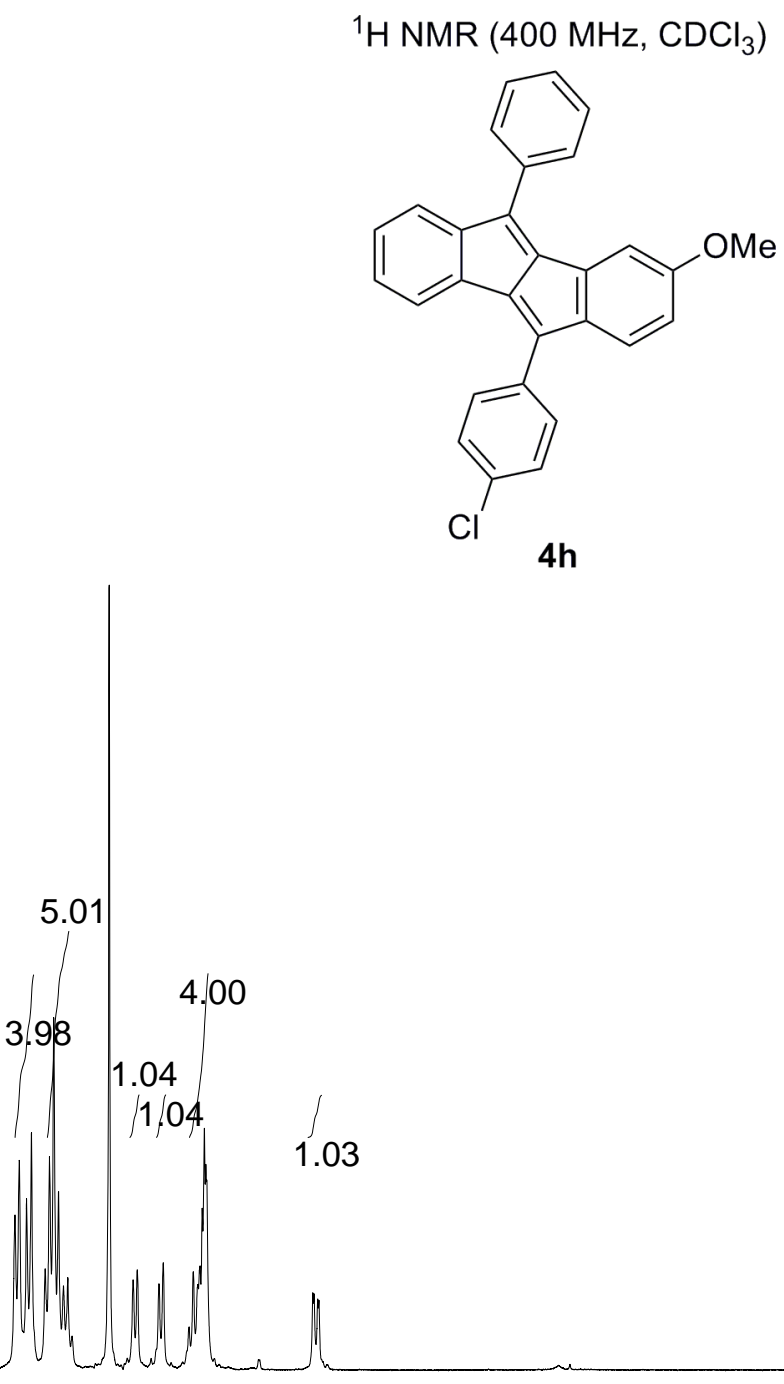

$4 h$ 
${ }^{13} \mathrm{C} \mathrm{NMR}\left(100 \mathrm{MHz}, \mathrm{CDCl}_{3}\right)$

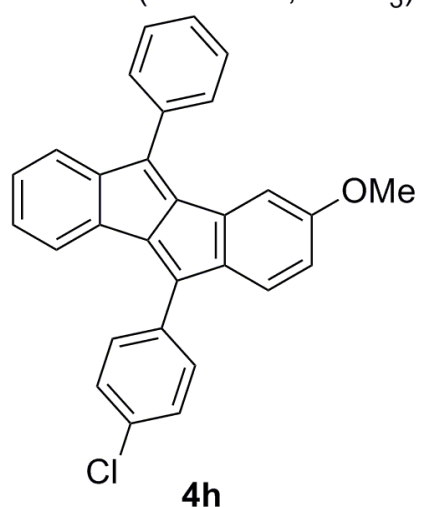


${ }^{1} \mathrm{H} \mathrm{NMR}\left(400 \mathrm{MHz}, \mathrm{CDCl}_{3}\right)$

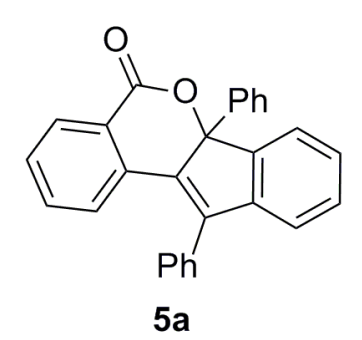

6.98

6.03

2.96

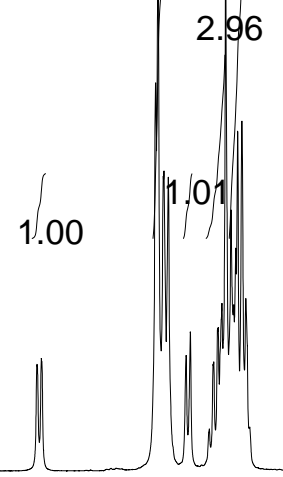




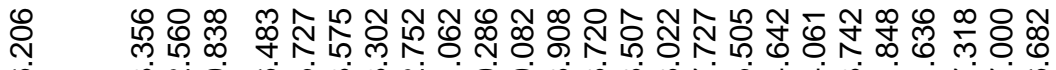

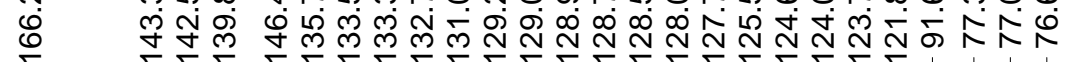

${ }^{13} \mathrm{C}$ NMR $\left(100 \mathrm{MHz}, \mathrm{CDCl}_{3}\right)$

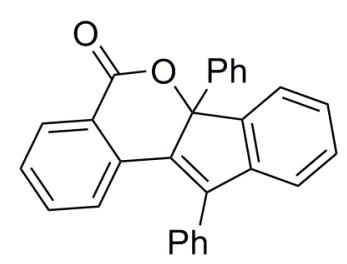

$5 a$

rid 
${ }^{1} \mathrm{H}$ NMR $\left(400 \mathrm{MHz}, \mathrm{CDCl}_{3}\right)$

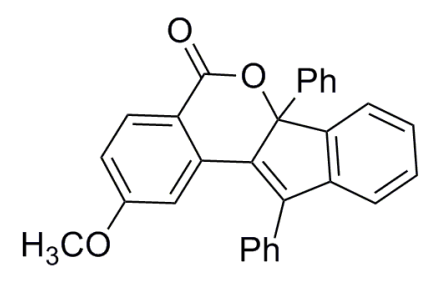

5b

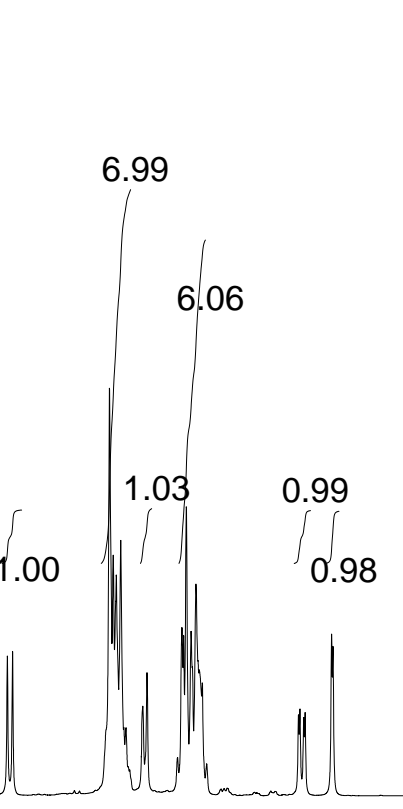

PPM 
${ }^{13} \mathrm{C}$ NMR $\left(100 \mathrm{MHz}, \mathrm{CDCl}_{3}\right)$

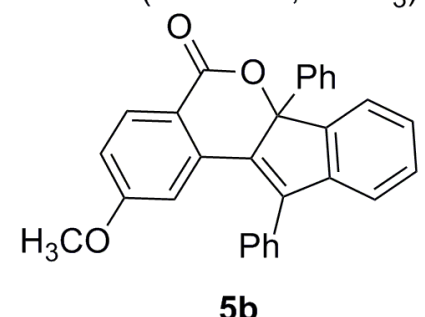

$5 b$ 


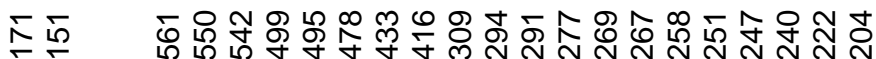

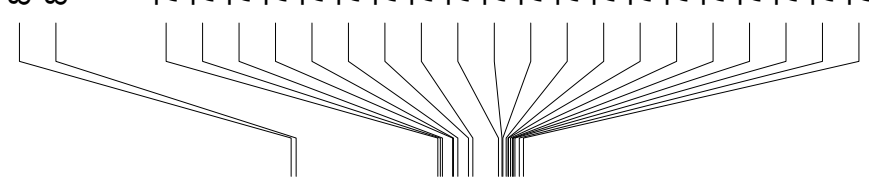

${ }^{1} \mathrm{H} \mathrm{NMR}\left(400 \mathrm{MHz}, \mathrm{CDCl}_{3}\right)$

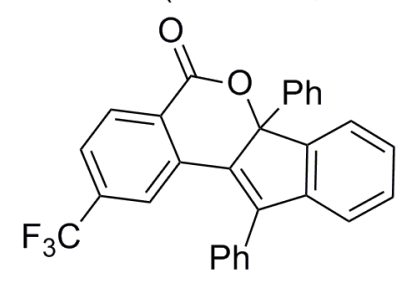

$5 c$

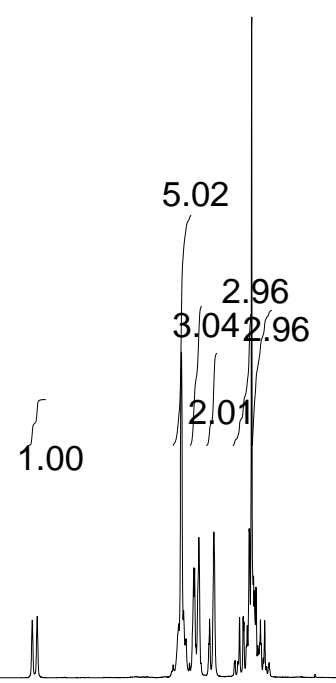




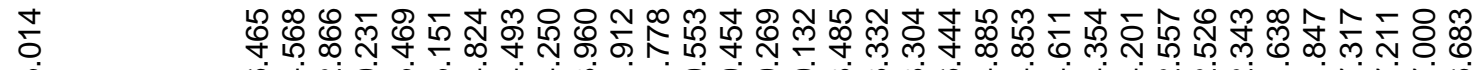

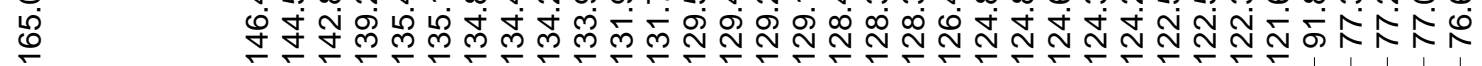

${ }^{13} \mathrm{C}$ NMR $\left(100 \mathrm{MHz}, \mathrm{CDCl}_{3}\right)$

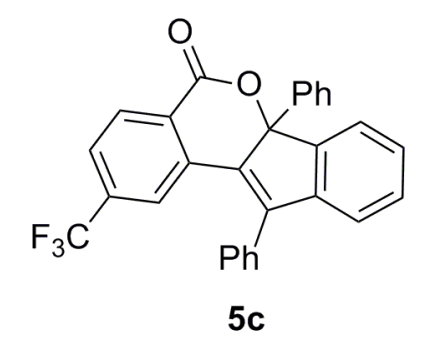


L
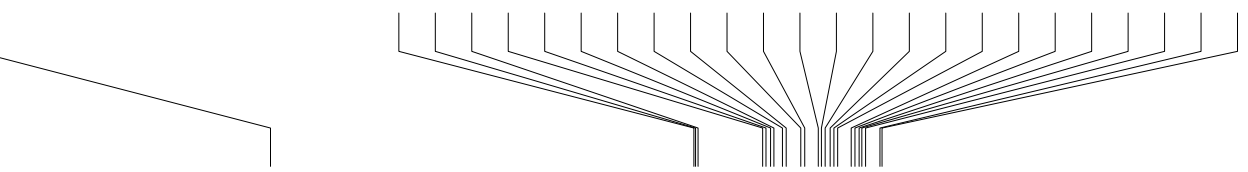

${ }^{1} \mathrm{H}$ NMR $\left(400 \mathrm{MHz}\right.$, DMSO- $\left.d_{6}\right)$

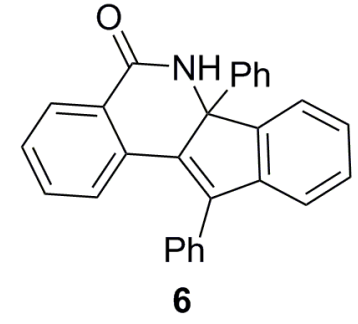

0.97

6.01

5.96

$\begin{array}{lr}1.99 \\ & \\ & \end{array}$ 


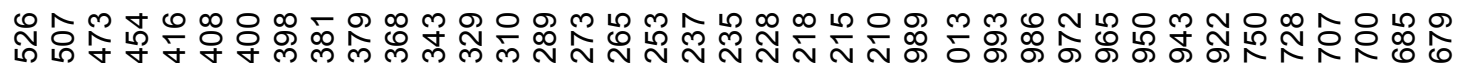

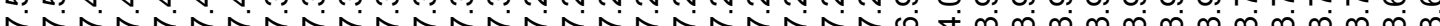
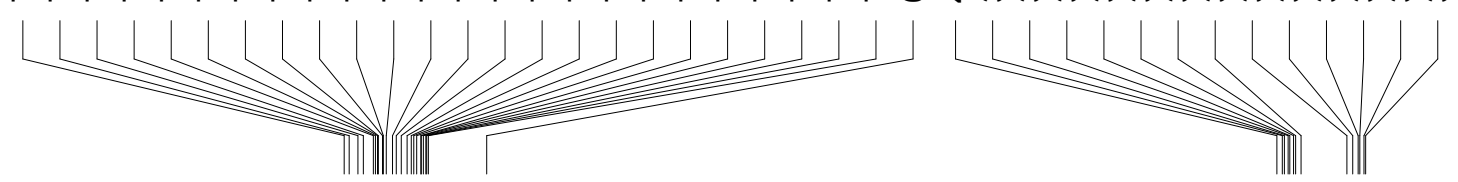

${ }^{1} \mathrm{H} \operatorname{NMR}\left(400 \mathrm{MHz}, \mathrm{CDCl}_{3}\right)$

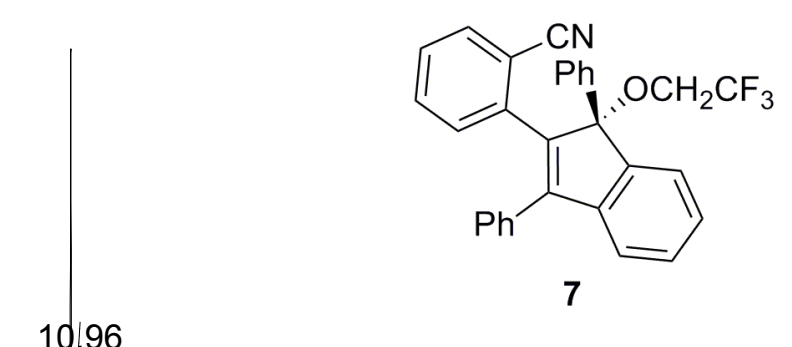

10.96 


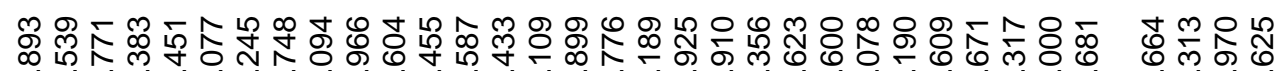

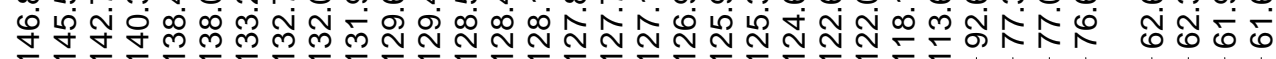

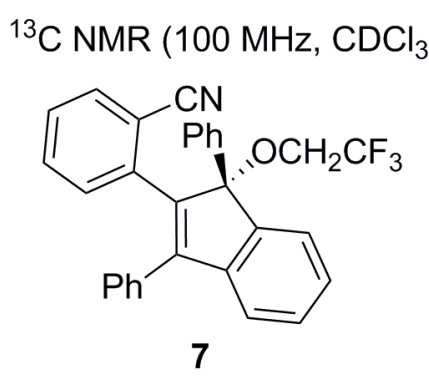


${ }^{1} \mathrm{H} \mathrm{NMR}\left(400 \mathrm{MHz}, \mathrm{CDCl}_{3}\right)$

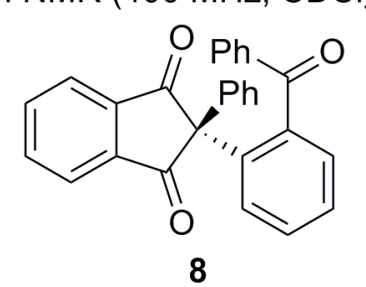


${ }^{13} \mathrm{C}$ NMR (100 MHz, $\mathrm{CDCl}_{3}$ )

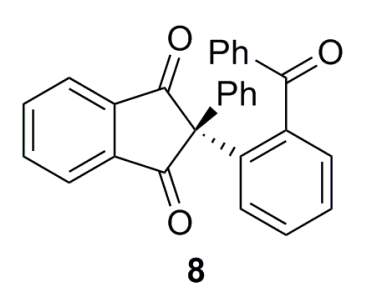


${ }^{1} \mathrm{H}$ NMR $\left(400 \mathrm{MHz}, \mathrm{CDCl}_{3}\right)$

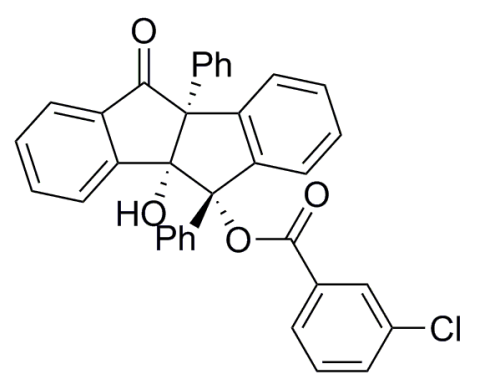

9

13.00

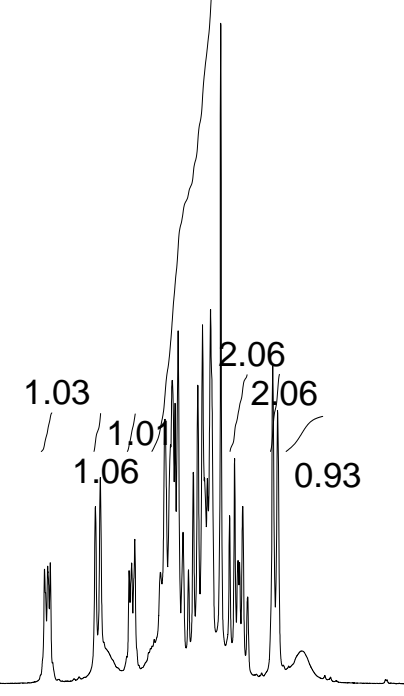

1.02 
${ }^{13} \mathrm{C} \mathrm{NMR}\left(100 \mathrm{MHz}, \mathrm{CDCl}_{3}\right)$

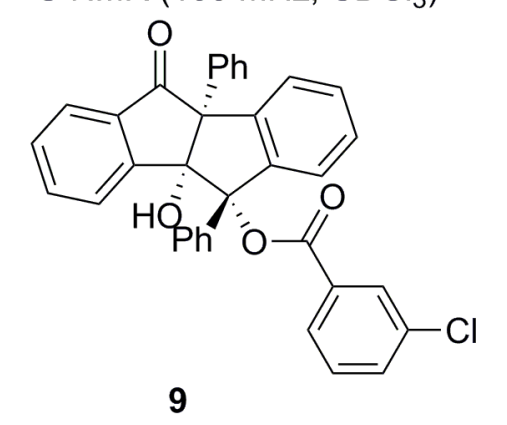


${ }^{1} \mathrm{H}$ NMR $\left(400 \mathrm{MHz}\right.$, DMSO- $\left.d_{6}\right)$

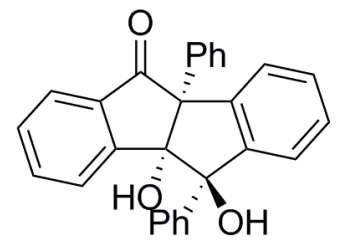

9-hydrolysis 
${ }^{13} \mathrm{C}$ NMR (100 MHz, DMSO- $\left.d_{6}\right)$

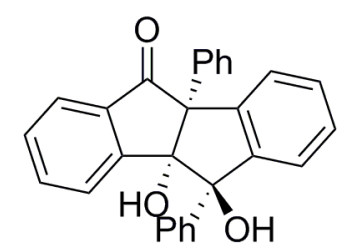

9-hydrolysis
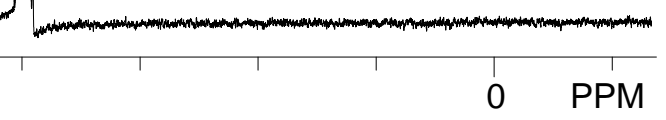

\section{S159}




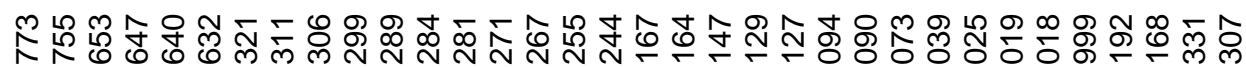

${ }^{1} \mathrm{H}$ NMR $\left(400 \mathrm{MHz}, \mathrm{CDCl}_{3}\right)$

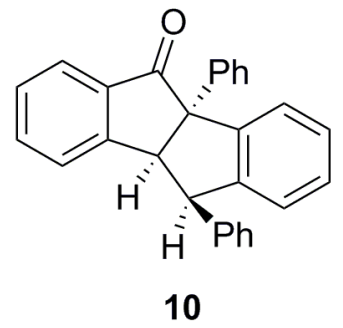

11.06 
${ }^{13} \mathrm{C}$ NMR $\left(100 \mathrm{MHz}, \mathrm{CDCl}_{3}\right)$

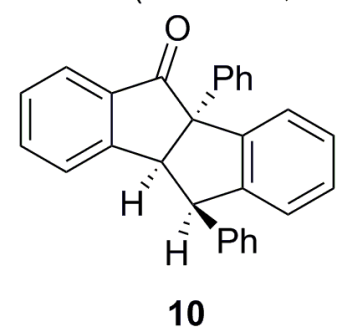


${ }^{1} \mathrm{H} \mathrm{NMR}\left(400 \mathrm{MHz}, \mathrm{CDCl}_{3}\right)$

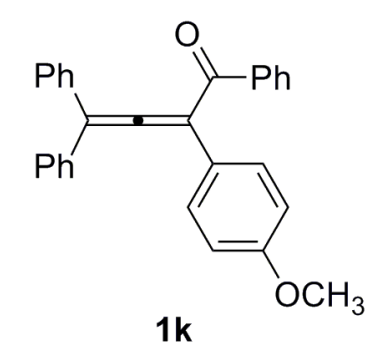

$1 \mathrm{k}$

6.05

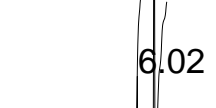

2.00

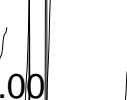

2.04 
${ }^{13} \mathrm{C}$ NMR $\left(100 \mathrm{MHz}, \mathrm{CDCl}_{3}\right)$

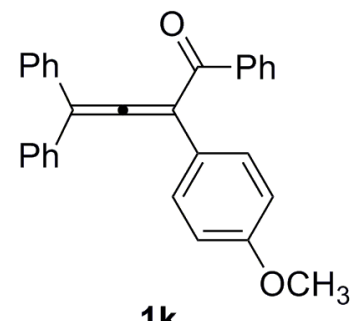


${ }^{1} \mathrm{H}$ NMR $\left(400 \mathrm{MHz}, \mathrm{CDCl}_{3}\right)$

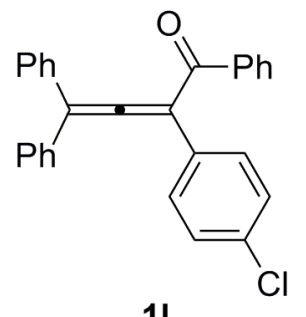

11

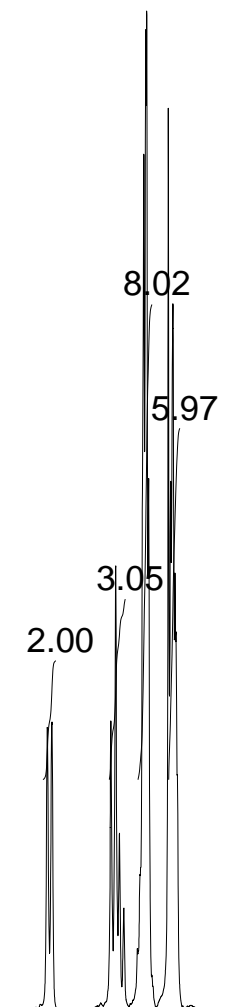

(1) 
${ }^{13} \mathrm{C}$ NMR $\left(100 \mathrm{MHz}, \mathrm{CDCl}_{3}\right)$

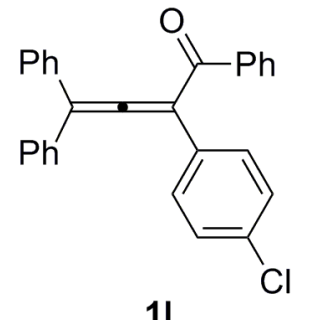

11

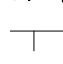


${ }^{1} \mathrm{H} \mathrm{NMR}\left(400 \mathrm{MHz}, \mathrm{CDCl}_{3}\right)$

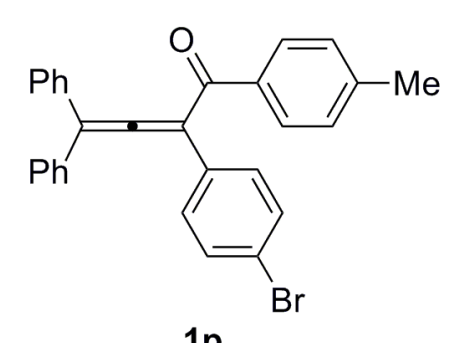

$1 p$

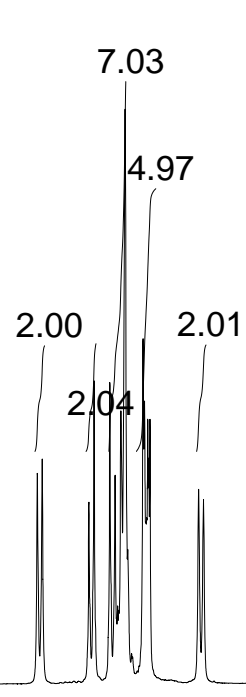


${ }^{13} \mathrm{C}$ NMR $\left(100 \mathrm{MHz}, \mathrm{CDCl}_{3}\right.$ )

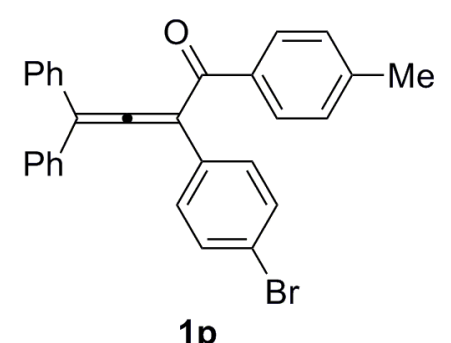

$1 p$ 
${ }^{1} \mathrm{H} \mathrm{NMR}\left(400 \mathrm{MHz}, \mathrm{CDCl}_{3}\right)$

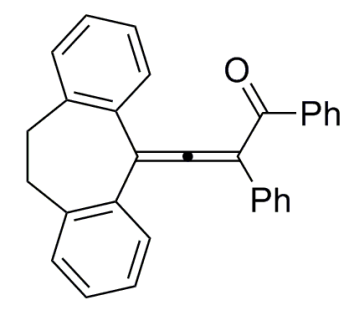

$1 \mathrm{~s}$ 
${ }^{13} \mathrm{C}$ NMR $\left(100 \mathrm{MHz}, \mathrm{CDCl}_{3}\right)$

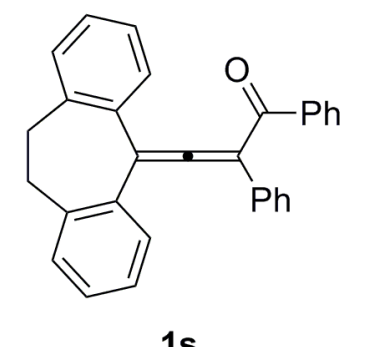

$1 \mathrm{~s}$ 

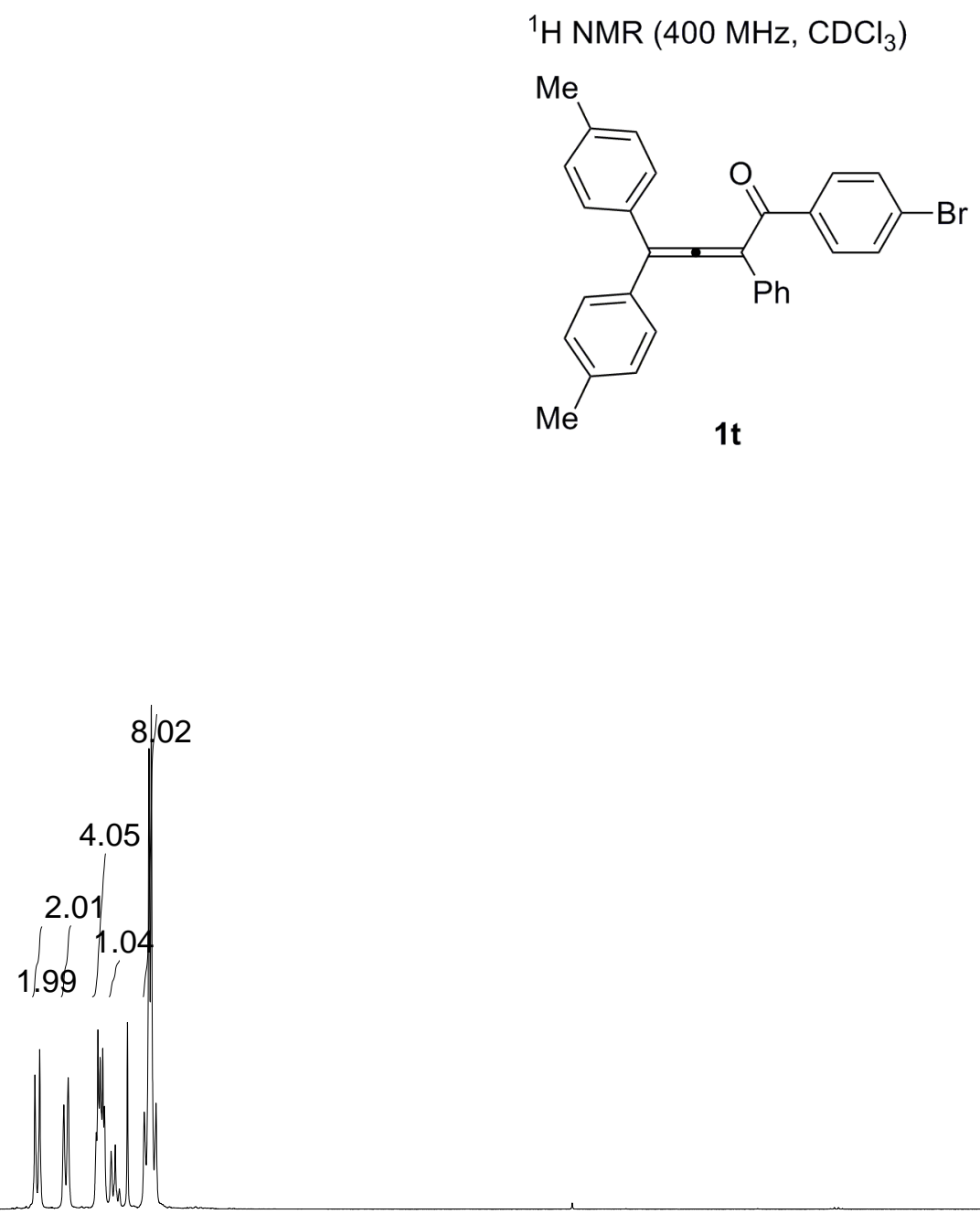

6.01 
${ }^{13} \mathrm{C}$ NMR $\left(100 \mathrm{MHz}, \mathrm{CDCl}_{3}\right)$

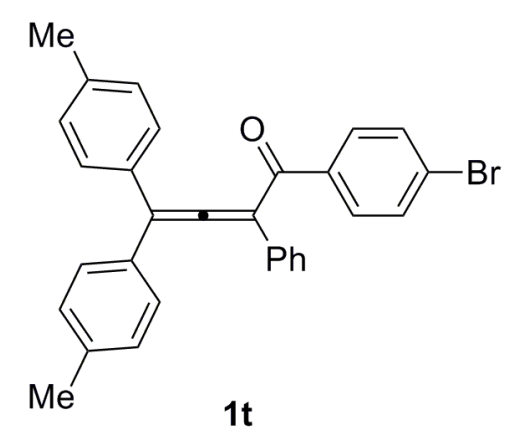

$1 t$ 


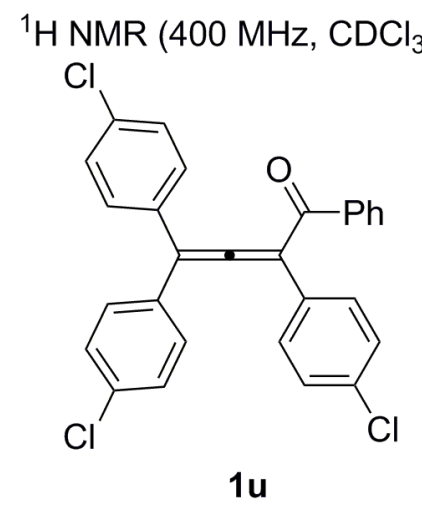

6.01

4.03

$2.00 \quad 1.99$

2.00

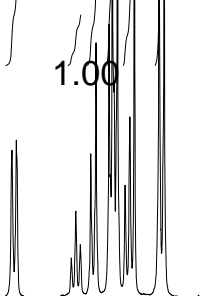


${ }^{13} \mathrm{C} \mathrm{NMR}\left(100 \mathrm{MHz}, \mathrm{CDCl}_{3}\right)$

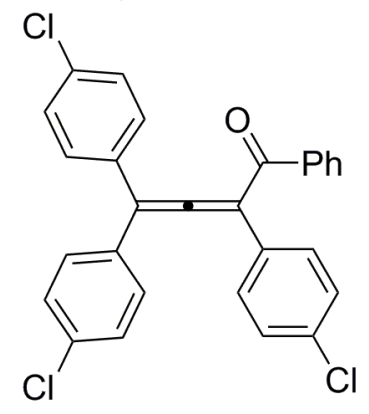

$1 \mathrm{u}$ 


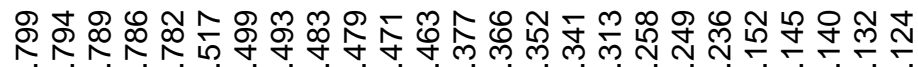

${ }^{1} \mathrm{H} \mathrm{NMR}\left(400 \mathrm{MHz}, \mathrm{CDCl}_{3}\right)$

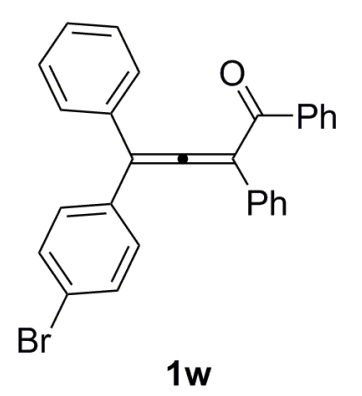

6.04

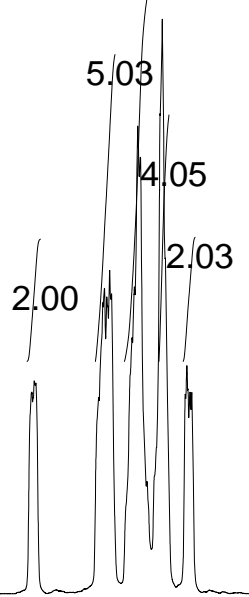


$\left.{ }^{13} \mathrm{C} \mathrm{NMR} \mathrm{(100} \mathrm{MHz,} \mathrm{CDCl}_{3}\right)$

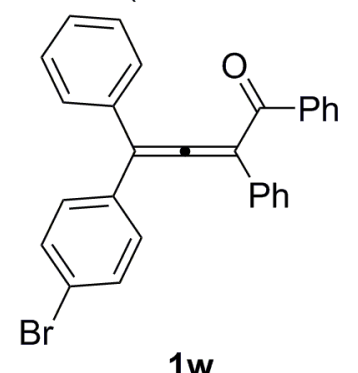

$1 w$ 
${ }^{1} \mathrm{H} \mathrm{NMR}\left(400 \mathrm{MHz}, \mathrm{CDCl}_{3}\right)$
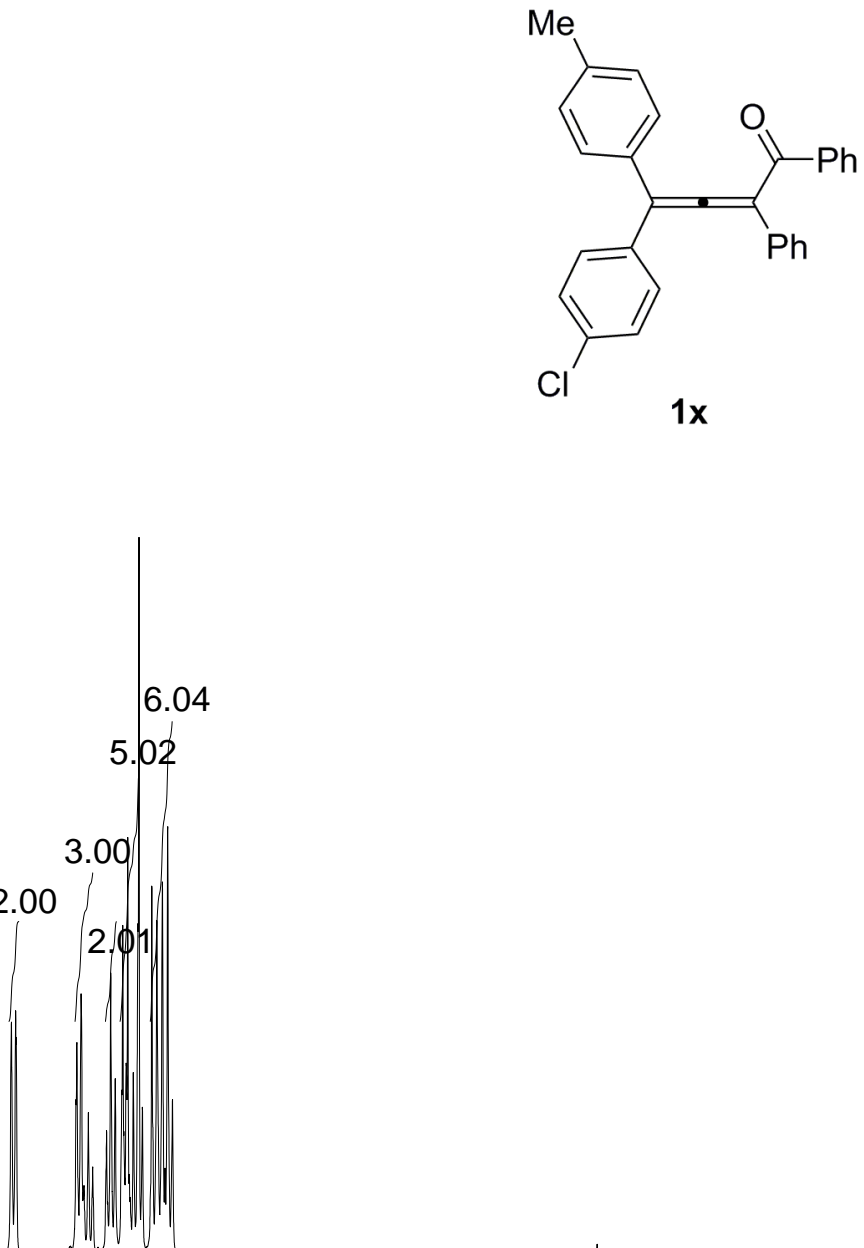

3.00 
${ }^{13} \mathrm{C}$ NMR $\left(100 \mathrm{MHz}, \mathrm{CDCl}_{3}\right)$

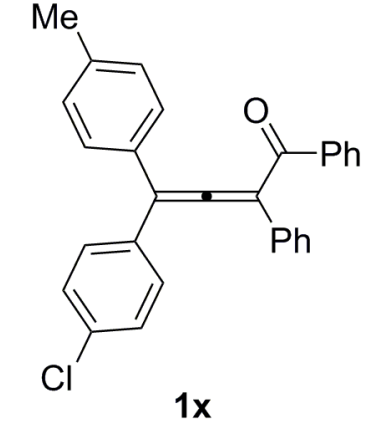



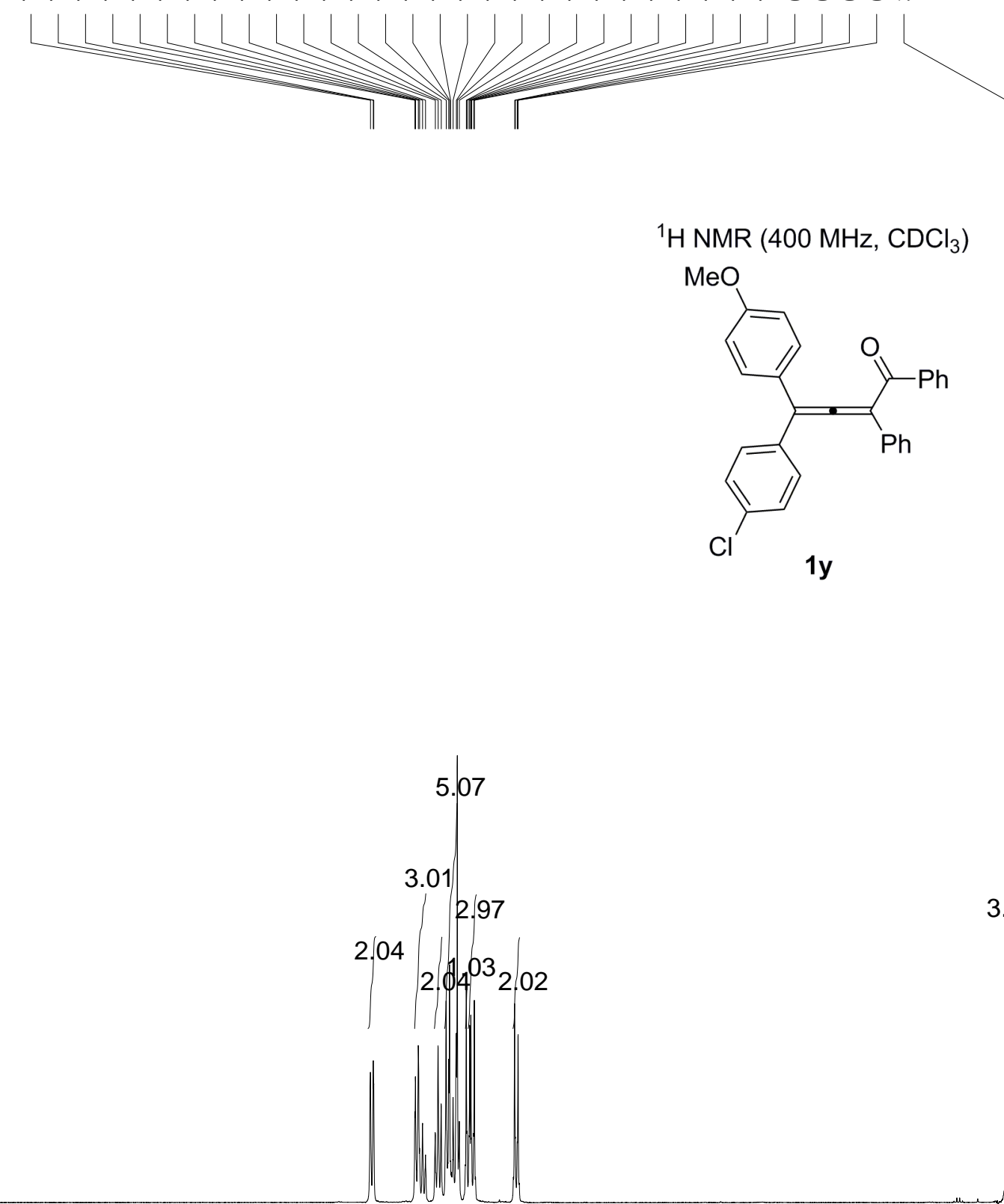
${ }^{13} \mathrm{C} \mathrm{NMR}\left(100 \mathrm{MHz}, \mathrm{CDCl}_{3}\right)$ $\mathrm{MeO}$

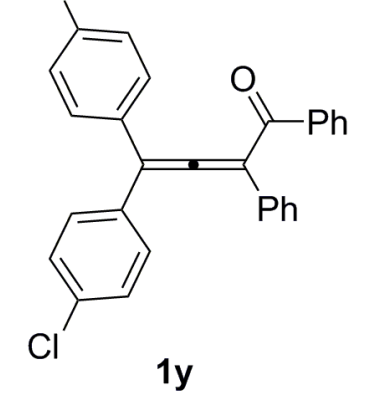


${ }^{1} \mathrm{H}$ NMR $\left(400 \mathrm{MHz}, \mathrm{CDCl}_{3}\right)$

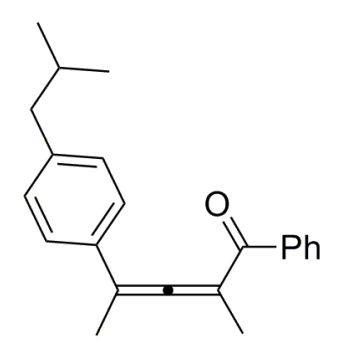

$1 z$

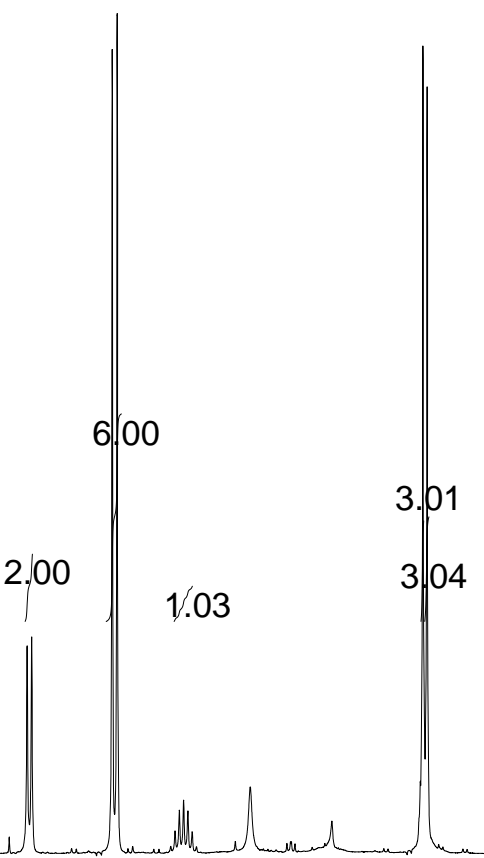


${ }^{13} \mathrm{C}$ NMR $\left(100 \mathrm{MHz}, \mathrm{CDCl}_{3}\right)$

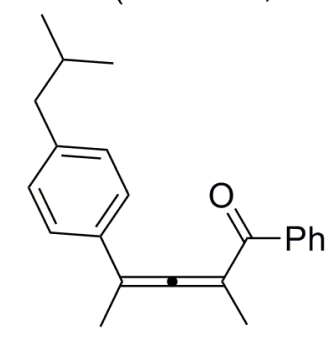

$1 z$ 